\title{
Altitudinal Variation of Metabolites, Mineral Elements and Antioxidant Activities of Rhodiola crenulata (Hook.f. \& Thomson) H.Ohba
}

\author{
Tingting Dong, Yueqi Sha, Hairong Liu and Liwei Sun *
}

National Engineering Laboratory for Tree Breeding, College of Biological Sciences and Technology, Beijing Forestry University, Beijing 100083, China; dongtingting187@163.com (T.D.); sha1285325561@163.com (Y.S.); Liuhairong03@163.com (H.L.)

* Correspondence: 1sun2013@bjfu.edu.cn; Tel.: +86-136-9139-7366

check for updates

Citation: Dong, T.; Sha, Y.; Liu, H.; Sun, L. Altitudinal Variation of

Metabolites, Mineral Elements and Antioxidant Activities of Rhodiola crenulata (Hook.f. \& Thomson) H.Ohba. Molecules 2021, 26, 7383. https://doi.org/10.3390/ molecules 26237383

Academic Editor:

Fernandez De Simon Brigida

Received: 3 November 2021

Accepted: 2 December 2021

Published: 5 December 2021

Publisher's Note: MDPI stays neutral with regard to jurisdictional claims in published maps and institutional affiliations.

Copyright: (c) 2021 by the authors. Licensee MDPI, Basel, Switzerland. This article is an open access article distributed under the terms and conditions of the Creative Commons Attribution (CC BY) license (https:/ / creativecommons.org/licenses/by/ $4.0 /)$.
Abstract: Rhodiola crenulata (Hook.f. \& Thomson) H.Ohba is an alpine medicinal plant that can survive in extreme high altitude environments. However, its changes to extreme high altitude are not yet clear. In this study, the response of Rhodiola crenulata to differences in altitude gradients was investigated through chemical, ICP-MS and metabolomic methods. A targeted study of Rhodiola crenulata growing at three vertical altitudes revealed that the contents of seven elements $\mathrm{Ca}, \mathrm{Sr}, \mathrm{B}$, $\mathrm{Mn}, \mathrm{Ni}, \mathrm{Cu}$, and $\mathrm{Cd}$, the phenolic components, the ascorbic acid, the ascorbic acid/dehydroascorbate ratio, and the antioxidant capacity were positively correlated with altitude, while the opposite was true for total ascorbic acid content. Furthermore, 1165 metabolites were identified: flavonoids (200), gallic acids (30), phenylpropanoids (237), amino acids (100), free fatty acids and glycerides (56), nucleotides (60), as well as other metabolites (482). The differential metabolite and biomarker analyses suggested that, with an increasing altitude: (1) the shikimic acid-phenylalanine-phenylpropanoidsflavonoids pathway was enhanced, with phenylpropanoids upregulating biomarkers much more than flavonoids; phenylpropanes and phenylmethanes upregulated, and phenylethanes downregulated; the upregulation of quercetin was especially significant in flavonoids; upregulation of condensed tannins and downregulation of hydrolyzed tannins; upregulation of shikimic acids and amino acids including phenylalanine. (2) significant upregulation of free fatty acids and downregulation of glycerides; and (3) upregulation of adenosine phosphates. Our findings provide new insights on the responses of Rhodiola crenulata to extreme high altitude adversity.

Keywords: rhodiola; high altitude; metabolite; element; change

\section{Introduction}

Rhodiola crenulata (Hook.f. \& Thomson) H.Ohba is an alpine medicinal species of the Rhodiola L., which is widely distributed in Asia, Europe, and the Americas. Several rhodiola species have been widely used in traditional medicine in Asia and Europe to improve human health and enhance physical fitness [1-4]. In China, Rhodiola crenulata has been recognized as a representative medicinal species, and its medicinal part rhizome is listed in the Chinese Pharmacopoeia [5,6]. Modern pharmacological studies have shown that Rhodiola crenulata is effective in enhancing immunity [7], delaying aging [8], preventing and treating cancer [9], protecting the heart [10], resisting plateau reactions [11]. In particular, it has been shown that Rhodiola crenulata has a variety of health benefits, including lung function and resistance to respiratory viral infections [12,13]. Rhodiola crenulata contains a variety of phytochemicals, including phytophenols (i.e., flavonoids + phenylpropanoids + tannins), gallic acids, amino acids, free fatty acids and glycerides, and nucleotides, and its health benefits are directly related to its phytochemicals $[4,14]$. Rhodiola crenulata grows mainly in Tibet, northwestern Yunnan, and western Sichuan in China, at altitudes of 2800-5600 $\mathrm{m}$ [15]. Therefore, it is able to adapt to the extreme high altitude environment, including low temperature, lack of oxygen and strong UV rays. 
Multiple types of components in plants are significantly linked to environmental stresses. Flavonoids, phenylpropanoids and tannins, collectively known as phytophenols (also known as plant phenolics), are important antioxidants in plant defense mechanisms [16-18]. Ascorbic acid is also an important antioxidant in plants against oxidative stress induced by adversity $[19,20]$. Flavonoids and phenylpropanoids possess the ability to resist a variety of adversity stresses such as UV radiation, low temperature, drought, or pathogen [21-26]. It has been shown that the amount and type of plant phenolics and ascorbic acid in the plant body would respond to a variety of adversities and play an important role in helping plants to withstand adversity stress [27-29]. Amino acid classes play an important physiological role in promoting plant growth and enhancing plant stress resistance in several ways [30-32]. Aminobutyric acid is associated with enhancing plant energy metabolism [33,34]. Phenylalanine, generated by the shikimic acid pathway, is then a key upstream intermediate in the synthesis of phenylpropanoids-flavonoids in plants, and is an important indicator of altered phenylpropanoids-flavonoids biosynthesis [35-37].

A wide range of mineral elements are closely related to improving plant stress resistance, i.e., $\mathrm{Ca}, \mathrm{Sr}, \mathrm{B}, \mathrm{Mn}, \mathrm{Ni}, \mathrm{Cu}$, and $\mathrm{Cd}$. Some studies have shown that they are closely related to the flavonoid composition and its content, the activities of antioxidant-related enzymes, and the cell wall synthesis in plants [38-46]. It has been reported that the roles of the above seven mineral elements in plants facilitate plants against adversities [38,47-52].

Other studies have shown that plant changes in response to adversity are also closely related to energy metabolism $[53,54]$. Fatty acids offer an essential source of energy for life activities in plants $[55,56]$, while adenosine phosphates, which are involved in ATP energy metabolism, supply a more straightforward energy utilization for plants [57]. It has also been suggested that fatty acid content and adenosine phosphates content change in a variety of plants under salt, low temperature or drought stress [58-62]. This indicates that adversity stress may cause changes in energy metabolism in different aspects.

However, these studies mostly focus on a single adversity and lack the investigation of integrated adversity, which is precisely in line with the actual situation of ecological environment. Therefore, such research is highly representative and urgent for application.

Alpine habitats exhibit specific properties of integrated adversities such as strong radiation, oxygen deprivation, alpine cold, freezing and thawing, as well as regionalspecific constraints on green vegetation cover [63-65]. Therefore, investigating plant changes in alpine habitats will provide a direct and effective way to understand plant change mechanisms from a holistic perspective and systemic cognitive level. Furthermore, it will provide strong support to improve green vegetation cover, crop resistance and crop quality in alpine and plateau areas, so as to adapt and improve the ecological environment for the practical development needs of human beings.

Our previous studies investigated the material change patterns of Rhodiola crenulata grown in the Sejila Mountains and Shannan in Tibet [14]. In this study, we investigated three vertical elevations $(3933,4249$ and $4531 \mathrm{~m}$ ) of Rhodiola crenulata from Dakazi Mountain (Ganzi Tibetan Autonomous Region, Sichuan, China), which is important for characterizing the effects of different elevations on Rhodiola crenulata in the same region. This is a rigorous investigation of the effects of different altitudes on the metabolites of Rhodiola crenulata in the same region and is highly relevant and representative. Furthermore, this study is more systematic and comprehensive in analyzing the changes of substances, which not only deepens the analysis of the changes of flavonoids, phenylpropanoids and amino acids, but also extends to seven new mineral elements, free fatty acids and glycerides and nucleotides related to the resistance.

\section{Results and Discussion}

\subsection{Effect of Altitude Gradient on Oxidative Stress Levels in Rhodiola crenulata}

Increased altitude leads to an intensification of integrated adversity stress consisting of multiple adversities such as UV radiation, low temperature, and hypoxia [63-65]. Excessive production of reactive oxygen species (ROS), such as $\mathrm{H}_{2} \mathrm{O}_{2}$ and $\mathrm{O}_{2}{ }^{--}$, leads to oxidative 
stress [66], which induces the emergence of protein carbonylation and lipid peroxidation as indicated by Malondialdehyde (MDA) [67-69], which together indicate the level of oxidative stress. The levels of oxidative stress and its adaptability in Rhodiola crenulata at different altitudes were investigated by measuring $\mathrm{H}_{2} \mathrm{O}_{2}$, protein carbonylation, $\mathrm{O}_{2}{ }^{--}$ and MDA levels. As shown in Figure $1 \mathrm{~A}, \mathrm{~B}, \mathrm{H}_{2} \mathrm{O}_{2}$ and protein carbonylation were stable at certain levels in all three elevation samples and did not increase significantly with elevation. As for $\mathrm{O}_{2}{ }^{-}$and MDA, both of them did not show any significant change between RC-L (3933 m) and RC-M (4249 m), while they showed a significant increase in RC-H (Figure 1C,D). The above results reveal that elevated altitude leads to increased oxidative stress and its level from the perspectives of $\mathrm{H}_{2} \mathrm{O}_{2}$, protein carbonylation, $\mathrm{O}_{2}{ }^{--}$ and MDA, to which Rhodiola crenulata exhibits an excellent resistance.
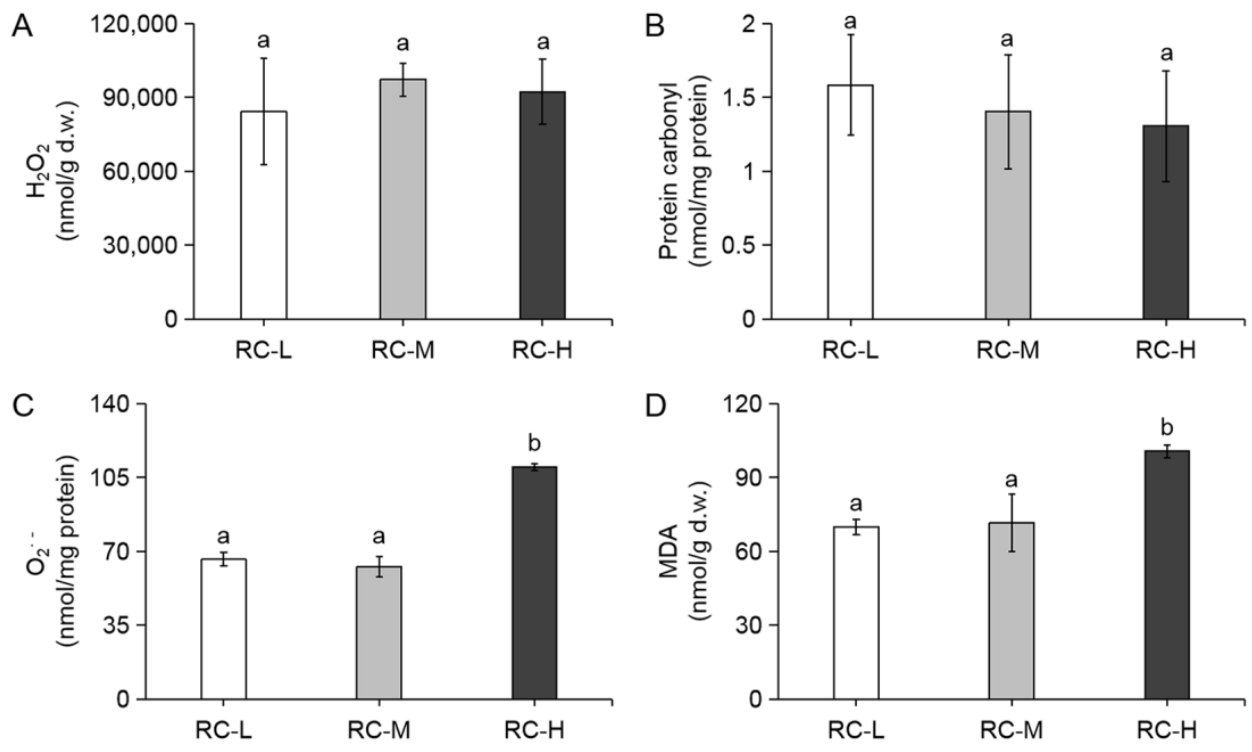

Figure 1. Oxidative states in rhizomes of Rhodiola crenulata (RC-L, RC-M, and RC-H) from different altitudes (3933, 4249 and $4531 \mathrm{~m}$ ), respectively. (A): $\mathrm{H}_{2} \mathrm{O}_{2} ;(\mathbf{B})$ : Protein carbonyl; (C): $\mathrm{O}_{2}{ }^{-{ }^{-}}$; (D): Malondialdehyde (MDA). Results are presented as the mean \pm SD of six independent experiments $(n=6)$. In each column, different letters $(a, b)$ mean significant differences between two groups $(p<$ $0.05)$ found via a $t$-test. Note: Rhodiola crenulata samples are presented according to the ascending order of altitude from left to right.

\subsection{Effect of Altitude Gradient on the Accumulation of Seven Mineral Elements in Rhodiola crenulata}

To characterize the response of Rhodiola crenulata to the altitude gradient in terms of the resistance-related mineral element accumulation, we measured the changes of accumulation of seven plant resistance-related mineral elements in Rhodiola crenulata rhizomes grown at different altitudes (3933, 4249 and $4531 \mathrm{~m}$ ), including $\mathrm{Ca}, \mathrm{Sr}, \mathrm{B}, \mathrm{Mn}, \mathrm{Ni}, \mathrm{Cu}$ and $\mathrm{Cd}$ (Figure 2). The results showed that the accumulation of these seven mineral elements in Rhodiola crenulata rhizome at 3933-4531 m showed an overall increase with altitude, i.e., RC-L < RC-M < RC-H. The accumulation of five elements, i.e., $\mathrm{Ca}, \mathrm{Sr}, \mathrm{B}$, $\mathrm{Mn}$, and $\mathrm{Ni}$, changed significantly with altitude in some altitude gradient zones. The accumulation of the remaining two elements, i.e., $\mathrm{Cu}$ and $\mathrm{Cd}$, exhibited a rising trend with increasing altitude. 


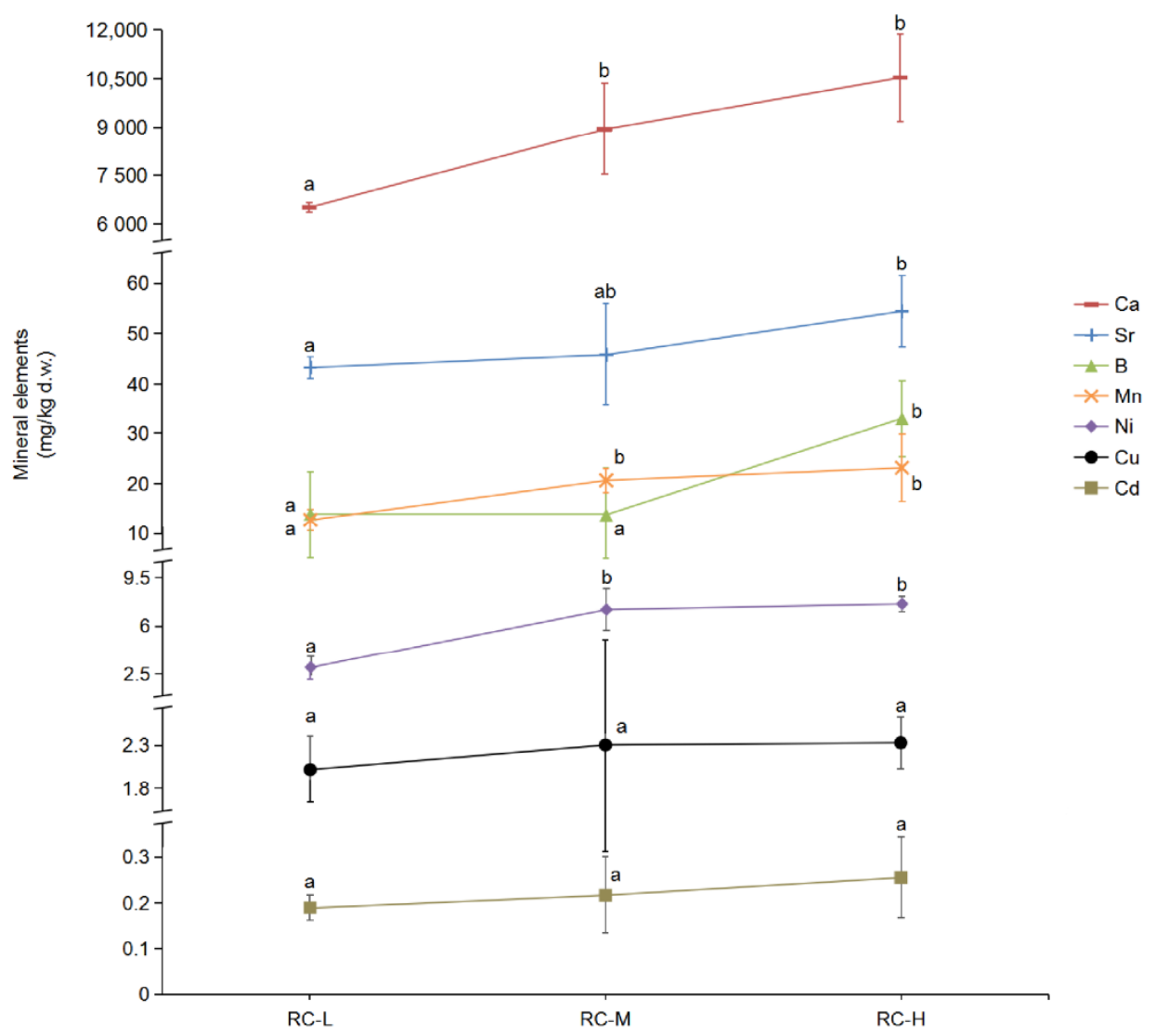

Figure 2. Accumulations of seven mineral elements in rhizomes of Rhodiola crenulata (RC-L, RC-M, and RC-H) from different altitudes (3933, 4249 and $4531 \mathrm{~m})$, respectively. Calcium (Ca); Strontium (Sr); Boron (B); Manganese (Mn); Nickel (Ni); Copper (Cu); Cadmium (Cd). Results are presented as the mean $\pm S D$ of four independent experiments $(n=4)$. Different letters $(a, b)$ mean significant differences between two groups $(p<0.05)$ found via a $t$-test. Note: Rhodiola crenulata samples are presented according to the ascending order of altitude from left to right.

A thorough analysis of the variation characteristics of the seven mineral elements with altitude revealed that the accumulation of $\mathrm{Ca}, \mathrm{Sr}, \mathrm{B}, \mathrm{Mn}$ and $\mathrm{Ni}$ increased significantly with altitude in some altitude gradient zones. Specifically, the accumulation of $\mathrm{Ca}, \mathrm{Mn}$, and $\mathrm{Ni}$ in the rhodiola rhizomes at both middle and high altitudes significantly increased compared to the lower altitude samples. This indicates that the altitude range from 3933-4249 m may contribute to the significant accumulation of $\mathrm{Ca}, \mathrm{Mn}$ and $\mathrm{Ni}$ in Rhodiola crenulata. The accumulation of $\mathrm{Sr}$ in Rhodiola crenulata rhizomes at high altitude (i.e., $4531 \mathrm{~m}$ ) was significantly higher than that in the low altitude (i.e., $3933 \mathrm{~m}$ ) sample, which indicates that the accumulation of $\mathrm{Sr}$ in Rhodiola crenulata rhizomes may be closely related to the wider altitude span. The accumulation of B in rhodiola rhizomes from high elevation significantly exceeded those from low and middle elevations, denoting that elevations from $4249-4531 \mathrm{~m}$ might favor accumulation of B in Rhodiola crenulata. The accumulation of $\mathrm{Cu}$ and $\mathrm{Cd}$ in Rhodiola crenulata rhizomes showed a general increasing trend with elevation from 3933-4531 m, suggesting that elevation may promote the accumulation of these two elements in Rhodiola crenulata rhizomes.

The above results suggest that elevation in the altitude of 3933-4531 m contributes to the accumulation of seven mineral elements, $\mathrm{Ca}, \mathrm{Sr}, \mathrm{B}, \mathrm{Mn}, \mathrm{Ni}, \mathrm{Cu}$ and $\mathrm{Cd}$, in Rhodiola crenulata rhizomes. Ca contributes to plant resistance by enhancing pectin methyl esterase activity and inhibiting pectin hydrolase (i.e., polygalacturonase and galactosidase) activity, which plays a role in the formation of plant cell walls [39,40]. Acuña et al. [38] showed a positive correlation between the concentration of antioxidant active compounds such as catechin and epicatechin in grapes and the content of elements such as $\mathrm{Sr}$ and $\mathrm{Mn}$. 
Hanaka et al. [43] found that the concentration of Sr in Glycine max was positively correlated with its isoflavone content. $\mathrm{B}$ attenuated oxidative stress induced by reactive oxygen species (e.g., $\mathrm{H}_{2} \mathrm{O}_{2}$ and $\mathrm{O}_{2}$ ) by enhancing the activity of antioxidant enzymes in the plant above ground [42]. $\mathrm{Mn}$ and $\mathrm{Cu}$ are metal auxiliary groups of superoxide dismutase (SOD), which have important effects on its activity of scavenging reactive oxygen species, such as $\mathrm{H}_{2} \mathrm{O}_{2}$ and $\mathrm{O}_{2}[45,46,52]$. Kovacik et al. [41] found that Ni accumulation in Matricaria chamomilla increased its total phenol and chlorogenic acid content. Han et al. [44] reported that the accumulation of $\mathrm{Cd}$ in pumpkin roots increased its superoxide dismutase, peroxidase, and catalase activities to remove reactive oxygen species.

Furthermore, Singh et al. [70] found that the concentrations of Ca in the roots of wild Rheum Emodi Wallr. that collected in October was $3684.0 \mathrm{mg} / \mathrm{kg}$ of d.w. for $3400 \mathrm{~m}$, which increased to $12,235.0 \mathrm{mg} / \mathrm{kg}$ of d.w. for $3600 \mathrm{~m}$. This report was consistent with our results for Ca $(6506.8 \mathrm{mg} / \mathrm{kg}$ of d.w. for $3933 \mathrm{~m} ; 10,540.5 \mathrm{mg} / \mathrm{kg}$ of d.w. for $4531 \mathrm{~m})$, indicating that altitude increase leaded to the increased accumulation of $\mathrm{Ca}$ in Rhodiola crenulata rhizomes. Negi et al. [71] reported the concentrations of $\mathrm{Mn}$ and $\mathrm{Cu}$ in the roots of wild Swertia speciosa (G. Don) that collected in September-October were 3.0 and $18.0 \mathrm{mg} / \mathrm{kg}$ of d.w. for 3400-3500 m, respectively, which increased to 16.0 and $19.0 \mathrm{mg} / \mathrm{kg}$ of d.w. for $4000 \mathrm{~m}$, respectively. Their report coincided with our results $(12.8$ and $2.0 \mathrm{mg} / \mathrm{kg}$ of d.w. of $\mathrm{Mn}$ and $\mathrm{Cu}$ for $3933 \mathrm{~m}$, respectively; 23.2 and $2.3 \mathrm{mg} / \mathrm{kg}$ of d.w. of $\mathrm{Mn}$ and $\mathrm{Cu}$ for $4531 \mathrm{~m}$, respectively), implying that accumulations of $\mathrm{Mn}$ and $\mathrm{Cu}$ in Rhodiola crenulata rhizomes were augmented with elevations.

Regarding to the remaining four elements (namely $\mathrm{Sr}, \mathrm{B}, \mathrm{Ni}$ and $\mathrm{Cd}$ ), to our best knowledge, we reported here for the first time their concentrations were increased with elevation rise in plants.

Combined with the above studies, we inferred that the increased concentrations of these seven mineral elements in Rhodiola crenulata rhizomes might strengthen the adaptation of Rhodiola crenulata to high altitude adversity in multiple fashions.

\subsection{Effects of Altitude Gradient on Phenolic Components Content, Ascorbic Acid Content and Antioxidant Capacity in Rhodiola crenulata}

To examine the response of Rhodiola crenulata to altitude gradient from the perspective of phenolic components and antioxidant capacity, we determined the changes of phenolic components content and antioxidant capacity in the rhizomes of Rhodiola crenulata grown at three altitudes $(3933,4249$ and $4531 \mathrm{~m})$. As shown in Figure 3 and Table 1, the total phenols, total flavonoids, total tannins and condensed tannins contents, and the antioxidant capacity of DPPH and ABTS showed a significant increase with altitude, i.e., RC-L $<$ RC-M $<\mathrm{RC}-\mathrm{H}$, and there was a significant difference between the three altitude samples compared with each other. Our results suggest that elevated altitude facilitates the accumulation and enhancement of phenolic components and antioxidant capacity in Rhodiola crenulata rhizomes. In addition, the positive correlation between DPPH and ABTS free radical removal ability and phenolic content was also suggested, and the positive correlation with total phenols, total flavonoids and condensed tannins content was particularly noticeable when the increasing trend between the three altitude samples was further examined. 

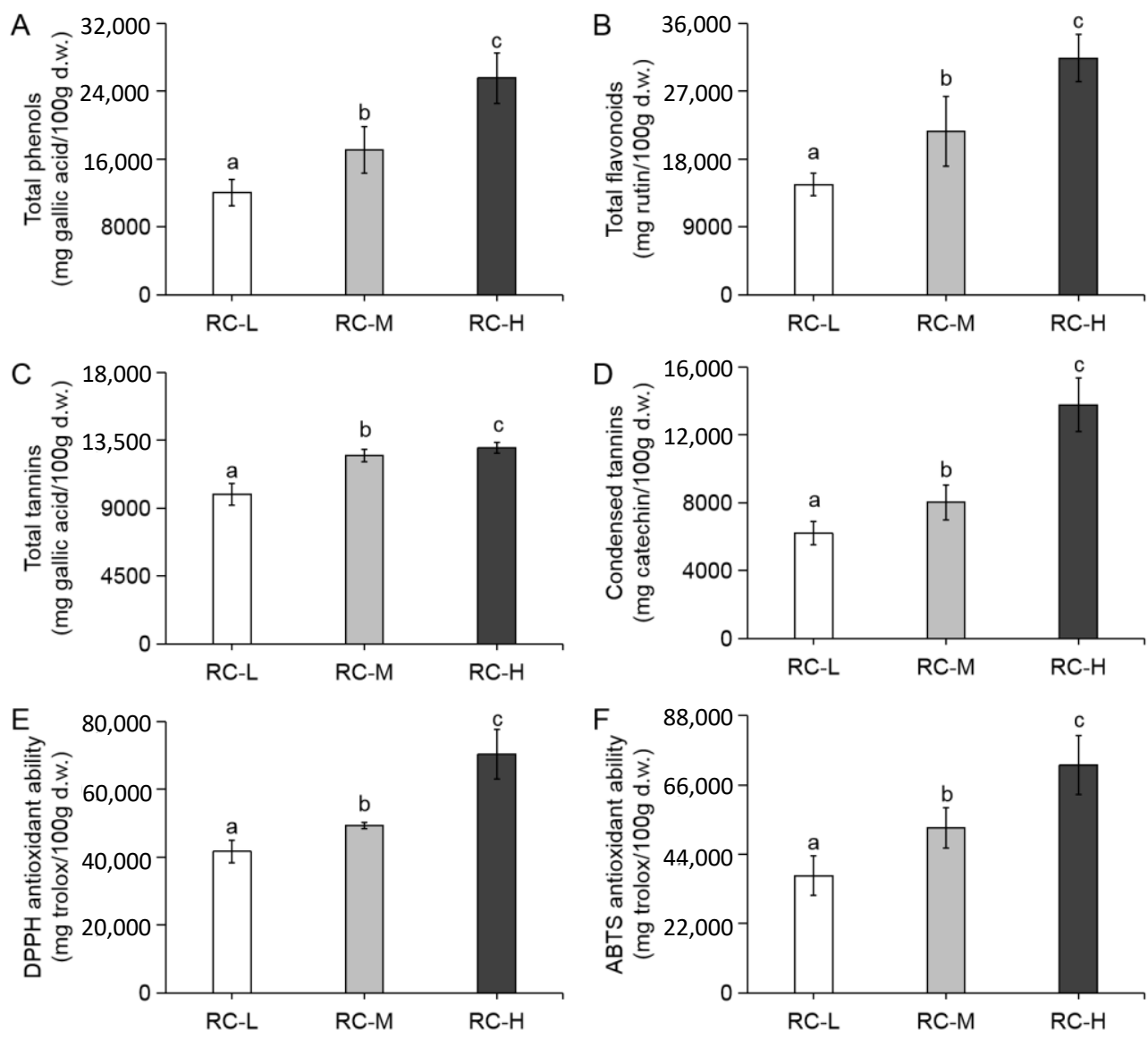

Figure 3. Characteristics of phenolic components and antioxidant capacity for rhizomes of Rhodiola crenulata (RC-L, RC-M, and RC-H) from different altitudes (3933, 4249 and $4531 \mathrm{~m}$ ), respectively. (A): Total phenols; (B): Total flavonoids; (C): Total tannins; (D): Condensed tannins; (E): DPPH antioxidant ability (DPPH is a abbreviation of 2,2-diphenyl-1-picrylhydrazyl); (F): ABTS antioxidant ability (ABTS is a abbreviation of (2,2'-azino-bis(3-ethylbenzothiazoline-6-sulphonic acid). Gallic acid was used as a standard for Total phenols and Total tannins. Rutin and Catechin were used as standards for Total flavonoids and Condensed tannins, respectively. Trolox was used as a standard for DPPH and ABTS antioxidant ability. Results are presented as the mean \pm SD of six independent experiments $(n=6)$ and are expressed as $\mathrm{mg}$ standard per $100 \mathrm{~g}$ d.w. of plant materials. In each column, different letters $(\mathrm{a}, \mathrm{b}, \mathrm{c})$ mean significant differences between two groups $(p<0.05)$ found via a $t$-test. Note: Rhodiola crenulata samples are presented according to the ascending order of altitude from left to right.

Table 1. Antioxidant activities for rhizomes of Rhodiola crenulata from different altitudes.

\begin{tabular}{ccc}
\hline Samples & IC $_{\mathbf{5 0}}(\boldsymbol{\mu g} / \mathbf{m L})$ DPPH & IC $_{\mathbf{5 0}}(\mu \mathrm{g} / \mathrm{mL})$ ABTS \\
\hline RC-L & 29.05 & 34.37 \\
RC-M & 20.06 & 24.16 \\
RC-H & 16.39 & 16.61
\end{tabular}

Note: RC-L, RC-M, and RC-H denotes rhizomes of Rhodiola crenulata from different altitudes $(3933,4249$ and $4531 \mathrm{~m})$, respectively.

To probe the pattern of ascorbic acid response to the altitude gradient, we then determined the ascorbic acid content, ascorbic acid/dehydroascorbate ratio and total ascorbic acid content in the rhizomes of Rhodiola crenulata grown at three altitudes (3933, 4249 and $4531 \mathrm{~m}$ ). As can be seen from Figure 4A,B, the ascorbic acid content and ascorbic acid/dehydroascorbate ratio (i.e., AsA/DHA) showed a significant increase with altitude, 
while the total ascorbic acid content showed a sharp decrease with elevation (Figure 4C). These results suggested a possible contribution of ascorbic acid (AsA) in the resistance of Rhodiola crenulata to stresses exacerbated by altitude, but the dramatic decrease in total ascorbic acid content suggested that the role of ascorbic acid might be limited.
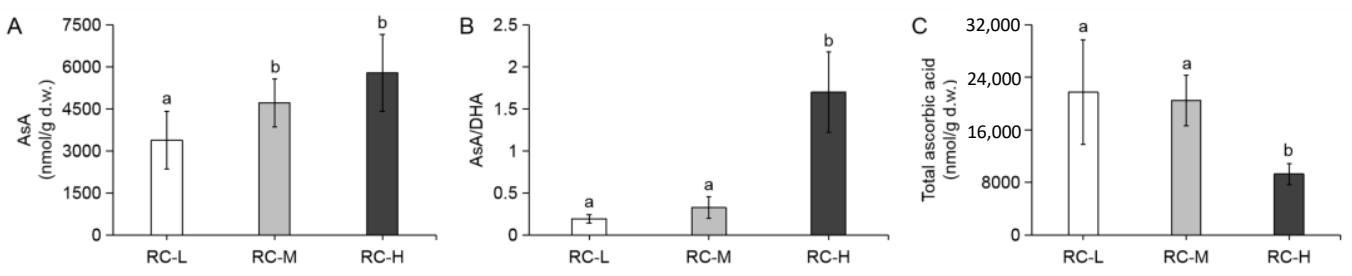

Figure 4. Contents of ascorbic acid in rhizomes of Rhodiola crenulata (RC-L, RC-M, and RC-H) from different altitudes (3933, 4249 and $4531 \mathrm{~m}$ ), respectively. (A): AsA (ascorbic acid); (B): AsA/DHA (ascorbic acid/dehydroascorbate); (C): Total ascorbic acid. Results are presented as the mean \pm SD of six independent experiments $(n=6)$. In each column, different letters $(a, b)$ mean significant difference between two groups $(p<0.05)$ found via a $t$-test. Note: Rhodiola crenulata samples are presented according to the ascending order of altitude from left to right.

The intensified UV-B radiation and low temperature stress at high altitude caused severe damage to the plant by introducing oxidative stress, which could be effectively counteracted by accumulating phenolic components to improve adaptive capacity so as to mitigate the adversity and survive better [72,73]. It has been shown that flavonoids, phenylpropanoids and tannins increase significantly in plants with elevation, which is consistent with our results [74-77].

Taken together, total ascorbic acid content in Rhodiola crenulata rhizomes at three altitudes (3933, 4249 and $4531 \mathrm{~m}$ ) significantly decreased with altitude gradient, while total phenols, total flavonoids, total tannins, and condensed tannins contents, and antioxidant capacity showed significant increase. This suggests that phenolic components may serve a more significant role in the response of Rhodiola crenulata to altitude gradient.

The results of Section 2.3 for phenolic components and their closely related antioxidant capacity with increasing altitude favorably support the finding that increasing altitude leads to an increase in oxidative stress and its level in Section 2.1. This fully demonstrates the significance of understanding how phenolic components respond to altitude gradients.

\subsection{Characteristics of Chemical Constituents of Rhodiola crenulata in Response to Altitude Gradient}

To systematically characterize and gain insight into the chemical changes influenced by altitude, a UPLC-QqQ-MS-based metabolomics approach was used to analyze metabolites in Rhodiola crenulata at altitude gradient (RC-L, altitude of $3933 \mathrm{~m}$; RC-M, altitude of $4249 \mathrm{~m}$; $\mathrm{RC}-\mathrm{H}$, altitude of $4531 \mathrm{~m}$ ).

A total of 1165 metabolites were identified, which were listed in Table S1, including flavonoids (200), gallic acid derivatives (30), phenylpropanoids (237), amino acids (100), free fatty acids and glycerides (56), nucleotides (60), and others (482), and then visualized as a heat map (Figure S1). It shows significant differences between three samples (RC-L, RC-M, and RC-H).

Unsupervised modeling using principal component analysis (PCA) was employed to assess the differences in the identified metabolites between the samples. The PCA plots for RC-L, RC-M, RC-H, and quality control (QC) samples in Figure 5A show that three samples were clustered into different regions, highlighting significant variations between RC-L, RC-M, and RC-H. Two principal components, PC1 and PC2, explained $60.0 \%$ of the total variance, manifesting a clear effect of altitude gradient on metabolites of Rhodiola crenulata. 

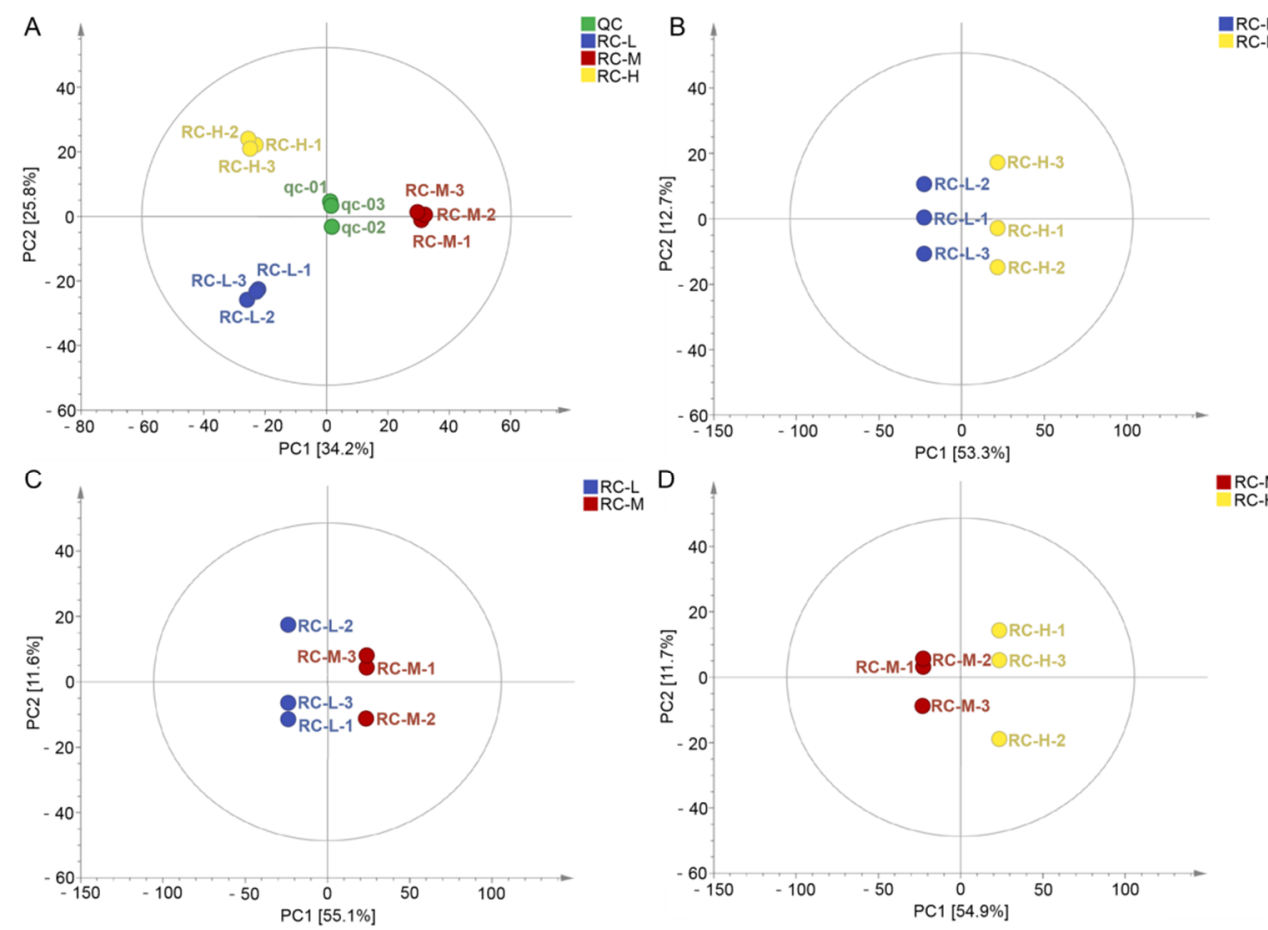

Figure 5. Principal component analysis (PCA) plots and orthogonal projections to latent structures discriminant analysis (OPLS-DA) of Rhodiola crenulata (RC-L, altitude of $3933 \mathrm{~m}$; RC-M, altitude of $4249 \mathrm{~m}$; RC-H, altitude of $4531 \mathrm{~m}$ ) with three repeats for respective sample. (A): Two-dimensional scatter plot of the PCA for RC-L, RC-M, RC-H, and the quality control (QC). The QC was a mixture of RC-L, RC-M, and RC-H; (B-D): Score scatter plots of the OPLS-DA for RC-H vs. RC-L, RC-M vs. RC-L and RC-H vs. RC-M.

An orthogonal projection to latent structures discriminant analysis (OPLS-DA) model was subsequently performed to compare metabolic characteristics of RC-L, RC-M, and RC-H. The OPLS-DA scatter scores of the three pairwise comparison groups are shown in Figure 5B-D, which show that RC-H and RC-L (Figure 5B), RC-M and RC-L (Figure 5C), and RC-H and RC-M (Figure 5D) were significantly distinguished in their pairwise comparisons.

Furthermore, the values of R2Y and Q2 by permutation tests of the OPLS-DA model confirmed the good credibility of individual models (Figure S2).

Next, differential metabolites were recognized and visualized as volcano maps based on the criteria of simultaneously meeting VIP $>1, \mid \log ^{2}$ (fold change) $\mid \geq 1$, and $p$-value $<0.05$ (Figure 6). For both pairwise comparisons, 267 (173 up- and 94 downregulated), 314 (207 upand 107 downregulated), and 335 (157 up- and 178 downregulated) differential metabolites were valid in distinguishing RC-H vs. RC-L, RC-M vs. RC-L, and RC-H vs. RC-M, respectively (also see Tables S2-S4). A heat map of changes of these differential metabolites in three rhodiola samples is shown in Figure S3. 
A

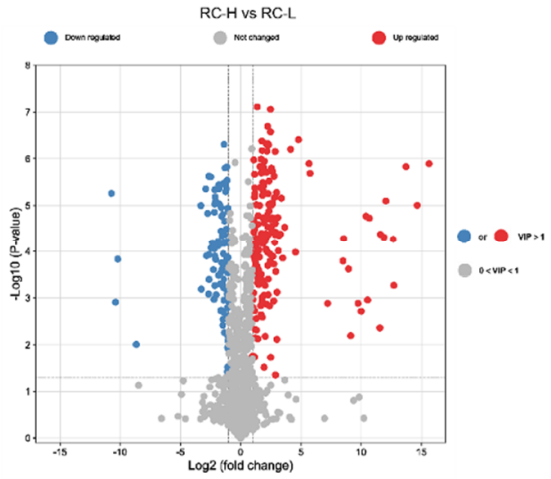

B

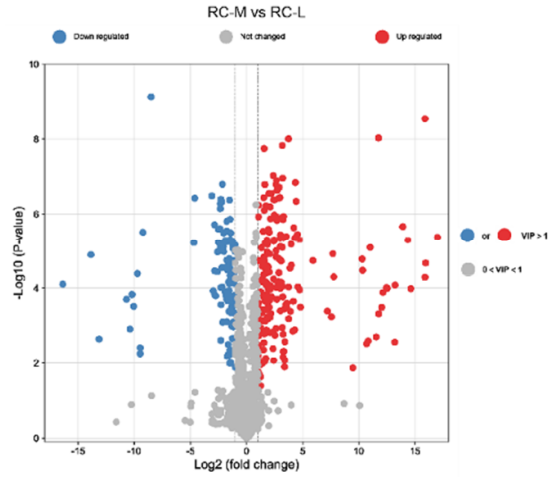

C

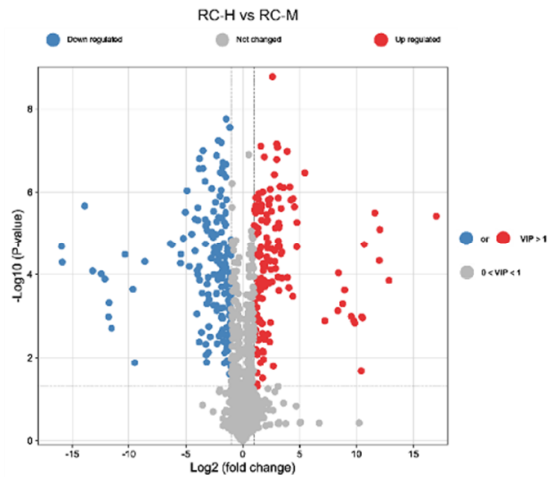

Figure 6. Differential metabolite analyses for RC-H vs. RC-L, RC-M vs. RC-L, and RC-H vs. RC-M with the criteria setting by simultaneously meeting variable importance in project (VIP) value $>1, \mid \log ^{2}$ (fold change) $\mid \geq 1$, and $p$-value $<0.05$, plotted by volcano maps. Red and blue dots denoted upregulated and downregulated differential metabolites, respectively; gray dots represented non-differential metabolites. Upregulation denotes that the content of a metabolite in RC increased with elevation between two altitudes, and the opposite for downregulation. (A): RC-H vs. RC-L; (B): RC-M vs. RC-L; (C): RC-H vs. RC-M.

Based on the changes in up- and downregulation of these differential metabolites, the specific alterations of Rhodiola crenulata metabolites in response to the elevation gradient from 3933-4531 m will be subsequently analyzed to explore the specific pattern of Rhodiola crenulata response to the elevation gradient.

\subsubsection{Biomarkers}

For the differential metabolites, 22, 35 and 28 that contributed significantly to the differentiation between RC-H and RC-L, RC-M and RC-L, and RC-H and RC-M, were first investigated (Table 2). Among these 22, 35 and 28 metabolites, 19, 23 and 17 of them were found exclusively in Rhodiola crenulata at relatively higher altitudes, suggesting that the production of these 19,23 and 17 were accompanied by elevations increasing from 3933-4531 m (RC-H vs. RC-L), 3933-4249 m (RC-M vs. RC-L), and 4249-4531 m (RC-H vs. RC-M). However, 3, 12 and 11 were only present at lower elevations in Rhodiola crenulata (correponding to RC-H vs. RC-L, RC-M vs. RC-L, and RC-H vs. RC-M), demonstrating that these 3,12 and 11 were closely related to the elevation decreases in their comparisons. Therefore, these 22, 35 and 28 metabolites could be used as biomarker to differentiate between Rhodiola crenulata grown at elevations ranging from 3933-4531 in three pairwise comparisons mentioned here.

Among these 19, 23 and 17 found exclusively in RC with higher altitude, 9, 7 and 8 belong to phenylpropanoids while 2, 2 and 1 are flavonoids, accounting for 47.37 vs. $10.53 \%$ of these 19 (RC-H vs. RC-L), 30.44 vs. $8.70 \%$ of these 23 (RC-M vs. RC-L), and 47.06 vs. $5.88 \%$ of these 17 (RC-H vs. RC-M), indicating that upregulation of phenylpropanoids biomarker dominated over those flavonoids counterparts. There is evidence that intensive UV radiation leads to the accumulation of phenylpropanoids in plants, which function as potent protectants against UV radiation, as well as low temperatures $[23,72,78,79]$. Based on the above findings and the common knowledge that an elevated altitude has severe effects on the ambient UV radiation and temperature, it is speculated that these 9, 7 and 8 phenylpropanoids may be greatly responsible for the resistance of Rhodiola crenulata to increased altitude-related stress, particularly for increased UV radiation, as well as a decrease in temperature, and they might be used as indicators to evaluate the resistance of Rhodiola crenulata to cope with altitude stresses. 
Table 2. Biomarkers for Rhodiola crenulata from altitude of 3933-4531 m (RC-H vs. RC-L; RC-M vs. RC-L; RC-H vs. RC-M).

\begin{tabular}{|c|c|c|c|c|c|c|c|}
\hline Metabolite & Category & $\begin{array}{l}\text { Fold Change } \\
\text { (RC-H vs. RC-L) }\end{array}$ & Type & $\begin{array}{l}\text { Fold Change } \\
\text { (RC-M vs. RC-L) }\end{array}$ & Type & $\begin{array}{c}\text { Fold Change } \\
\text { (RC-H vs. } \\
\text { RC-M) }\end{array}$ & Type \\
\hline Quercetin-3-O-rutinoside (Rutin) & Flavonoids & $50,814.82$ & $\mathrm{Up}$ & $60,233.70$ & $\mathrm{Up}$ & $\mathrm{N} / \mathrm{A}$ & $\mathrm{N} / \mathrm{A}$ \\
\hline 1-Methylpiperidine-2-carboxylic acid & Others & $\mathrm{N} / \mathrm{A}$ & $\mathrm{N} / \mathrm{A}$ & $63,187.78$ & Up & $1.58258 \mathrm{E}-05$ & Down \\
\hline 5-O-Caffeoylshikimic acid & Phenylpropanoids & $\mathrm{N} / \mathrm{A}$ & $\mathrm{N} / \mathrm{A}$ & $60,226.67$ & Up & $1.66039 \mathrm{E}-05$ & Down \\
\hline Rhododendrol & Phenylpropanoids & $25,852.22$ & Up & $25,319.26$ & Up & N/A & $\mathrm{N} / \mathrm{A}$ \\
\hline 7-O-Methxyl Quercetin (Rhamnetin) & Flavonoids & $13,547.41$ & Up & $130,288.89$ & Up & $\mathrm{N} / \mathrm{A}$ & $\mathrm{N} / \mathrm{A}$ \\
\hline LysoPC 19:2 & Others & $\mathrm{N} / \mathrm{A}$ & $\mathrm{N} / \mathrm{A}$ & $15,415.56$ & Up & $6.48695 \mathrm{E}-05$ & Down \\
\hline Phenylacetylglycine & Amino acids & $\mathrm{N} / \mathrm{A}$ & $\mathrm{N} / \mathrm{A}$ & 9512.74 & Up & 0.00011 & Down \\
\hline $\begin{array}{l}\text { 2,3,4,5,6-pentahydroxyhexyl } \\
\text { 2-hydroxybenzoate }\end{array}$ & Phenylpropanoids & 6782.52 & Up & 4217.04 & Up & $\mathrm{N} / \mathrm{A}$ & $\mathrm{N} / \mathrm{A}$ \\
\hline Catalposide & Others & $\mathrm{N} / \mathrm{A}$ & $\mathrm{N} / \mathrm{A}$ & 3510.22 & Up & 0.00029 & Down \\
\hline$N$-Acetyl-D-glucosamine-1-phosphate & Others & 6525.93 & $\mathrm{Up}$ & 5938.93 & Up & $\mathrm{N} / \mathrm{A}$ & $\mathrm{N} / \mathrm{A}$ \\
\hline LysoPC 20:1 & Others & $\mathrm{N} / \mathrm{A}$ & N/A & 5650.63 & Up & 0.00018 & Down \\
\hline 3-(3-Hydroxyphenyl)-propionate acid & Phenylpropanoids & $\mathrm{N} / \mathrm{A}$ & $\mathrm{N} / \mathrm{A}$ & 4544.74 & Up & 0.00022 & Down \\
\hline N-Acetyl-L-methionine & Amino acids & 4257.63 & Up & $\mathrm{N} / \mathrm{A}$ & $\mathrm{N} / \mathrm{A}$ & 4257.63 & Up \\
\hline S-(5'-Adenosy)-L-homocysteine & Amino acids & 3839.48 & Up & $\mathrm{N} / \mathrm{A}$ & $\mathrm{N} / \mathrm{A}$ & $\mathrm{N} / \mathrm{A}$ & $\mathrm{N} / \mathrm{A}$ \\
\hline Tryptamine & Others & 3096.93 & $\mathrm{Up}$ & $20,954.07$ & Up & $\mathrm{N} / \mathrm{A}$ & $\mathrm{N} / \mathrm{A}$ \\
\hline 3,5-Di-O-galloylshikimic acid & Phenylpropanoids & 3020.26 & $\mathrm{Up}$ & 9381.78 & $\mathrm{Up}$ & $\mathrm{N} / \mathrm{A}$ & $\mathrm{N} / \mathrm{A}$ \\
\hline Caffeoylbenzoyltartaric acid & Phenylpropanoids & 1654.26 & Up & $\mathrm{N} / \mathrm{A}$ & N/A & 1654.26 & Up \\
\hline 1,2-O-Diferuloylglycerol & Phenylpropanoids & 1494.74 & Up & $\mathrm{N} / \mathrm{A}$ & N/A & 1494.74 & Up \\
\hline 3-Aminosalicylic acid & Others & $\mathrm{N} / \mathrm{A}$ & N/A & $\mathrm{N} / \mathrm{A}$ & $\mathrm{N} / \mathrm{A}$ & 1415.11 & $\mathrm{Up}$ \\
\hline (5-L-Glutamyl)-L-amino acid & Amino acids & 1368.89 & Up & 1649.89 & Up & $\mathrm{N} / \mathrm{A}$ & $\mathrm{N} / \mathrm{A}$ \\
\hline LysoPC 18:4 & Others & $\mathrm{N} / \mathrm{A}$ & $\mathrm{N} / \mathrm{A}$ & 1302.52 & Up & 0.0008 & Down \\
\hline Phthalic acid & Others & 1038.84 & Up & 1834.74 & Up & $\mathrm{N} / \mathrm{A}$ & N/A \\
\hline Chlorogenic acid methyl ester & Phenylpropanoids & 868.57 & Up & $\mathrm{N} / \mathrm{A}$ & $\mathrm{N} / \mathrm{A}$ & 868.57 & Up \\
\hline Sinapoyl malate & Phenylpropanoids & 565.92 & $\mathrm{Up}$ & 3459.89 & Up & $\mathrm{N} / \mathrm{A}$ & N/A \\
\hline Scopoletin-7-O-glucoside (Scopolin) & Phenylpropanoids & $\mathrm{N} / \mathrm{A}$ & $\mathrm{N} / \mathrm{A}$ & 2996.11 & Up & 0.0003 & Down \\
\hline 4-Hydroxybenzyl Alcohol & Phenylpropanoids & 497.65 & Up & $\mathrm{N} / \mathrm{A}$ & $\mathrm{N} / \mathrm{A}$ & 497.65 & Up \\
\hline 9,10-Epoxyoctadecanoic Acid & $\begin{array}{l}\text { Free fatty acids } \\
\text { and glycerides }\end{array}$ & 381.88 & Up & 1260.63 & Up & $\mathrm{N} / \mathrm{A}$ & N/A \\
\hline Indole 3-acetic acid (IAA) & Others & $\mathrm{N} / \mathrm{A}$ & $\mathrm{N} / \mathrm{A}$ & 716.29 & $\mathrm{Up}$ & 0.0014 & Down \\
\hline $\begin{array}{c}\text { Cis-4,7,10,13,16,19-Docosahexaenoic } \\
\text { Acid }\end{array}$ & $\begin{array}{l}\text { Free fatty acids } \\
\text { and glycerides }\end{array}$ & $\mathrm{N} / \mathrm{A}$ & $\mathrm{N} / \mathrm{A}$ & 185.51 & Up & $\mathrm{N} / \mathrm{A}$ & $\mathrm{N} / \mathrm{A}$ \\
\hline 5,7-Dimethoxycoumarin & Phenylpropanoids & $\mathrm{N} / \mathrm{A}$ & $\mathrm{N} / \mathrm{A}$ & 0.0028 & Down & 445.73 & $\mathrm{Up}$ \\
\hline Isoeugenol & Phenylpropanoids & $\mathrm{N} / \mathrm{A}$ & $\mathrm{N} / \mathrm{A}$ & 0.0017 & Down & 345.22 & Up \\
\hline $\begin{array}{l}\text { 1- } \alpha \text {-Linolenoyl-glycerol-3-O- } \\
\text { glucoside }\end{array}$ & $\begin{array}{l}\text { Free fatty acids } \\
\text { and glycerides }\end{array}$ & $\mathrm{N} / \mathrm{A}$ & $\mathrm{N} / \mathrm{A}$ & 0.0014 & Down & 330.92 & Up \\
\hline 3-Indolepropionic acid & Others & $\mathrm{N} / \mathrm{A}$ & $\mathrm{N} / \mathrm{A}$ & 0.0014 & Down & 3134.78 & Up \\
\hline 3-O-p-Coumaroylquinic acid & Phenylpropanoids & $\mathrm{N} / \mathrm{A}$ & $\mathrm{N} / \mathrm{A}$ & 0.0012 & Down & 1371.50 & Up \\
\hline 2'-Deoxyuridine & Nucleotides & $\mathrm{N} / \mathrm{A}$ & $\mathrm{N} / \mathrm{A}$ & 0.0009 & Down & 748.40 & Up \\
\hline $\mathrm{N}$-(3-Indolylacetyl)-L-alanine & Amino acids & 365.44 & Up & 2071.70 & $\mathrm{Up}$ & $\mathrm{N} / \mathrm{A}$ & N/A \\
\hline Methyl caffeate & Phenylpropanoids & 147.26 & Up & $\mathrm{N} / \mathrm{A}$ & $\mathrm{N} / \mathrm{A}$ & 147.26 & Up \\
\hline Clove chromone & Others & 0.00085 & Down & 0.0009 & Down & $\mathrm{N} / \mathrm{A}$ & $\mathrm{N} / \mathrm{A}$ \\
\hline LysoPE $15: 0(2 n$ isomer $)$ & Others & 0.00075 & Down & 0.0008 & Down & $\mathrm{N} / \mathrm{A}$ & $\mathrm{N} / \mathrm{A}$ \\
\hline 4-Pyridoxic acid-O-glucoside & Others & $\mathrm{N} / \mathrm{A}$ & $\mathrm{N} / \mathrm{A}$ & 0.0006 & Down & 931.35 & Up \\
\hline Quercetin-3-O-(6"'-galloyl)galactoside & Flavonoids & $\mathrm{N} / \mathrm{A}$ & N/A & 0.0001 & Down & 7396.52 & $\mathrm{Up}$ \\
\hline 2-Aminopurine & Nucleotides & $\mathrm{N} / \mathrm{A}$ & $\mathrm{N} / \mathrm{A}$ & 6.617E-05 & Down & 4106.22 & Up \\
\hline 1,6-Di-O-Galloyl-D-Glucose & $\begin{array}{l}\text { Gallic acid } \\
\text { derivatives }\end{array}$ & $\mathrm{N} / \mathrm{A}$ & $\mathrm{N} / \mathrm{A}$ & $1.17381 \mathrm{E}-05$ & Down & $131,281.48$ & $\mathrm{Up}$ \\
\hline LysoPC 15:0 & Others & 0.00059 & Down & $\mathrm{N} / \mathrm{A}$ & $\mathrm{N} / \mathrm{A}$ & 0.00028 & Down \\
\hline
\end{tabular}

Note: 1. Upregulation denotes that the content of a metabolite in RC exhibited a significant increase compared to that of corresponding low altitude. Conversely, downregulation indicates the content of a metabolite in RC is significantly decreased compared with the one in that of corresponding low altitude; 2. Biomarkers were identified by the high abundance of certain metabolites in a Rhodiola sample versus zero abundance (set by $9.00 \mathrm{cps}$ ) of the corresponding metabolite in another Rhodiola sample; 3. N/A means Not Applicable.

\subsubsection{Six Categories of Differential Metabolites}

\section{Flavonoids}

Among the qualitative analysis of 200 flavonoids, 37 (31 upregulated vs. 6 downregulated, $83.78 \%$ upregulation rate, for RC-H vs. RC-L), 56 (43 upregulated vs. 13 downregulated, $76.79 \%$ upregulation rate, for RC-M vs. RC-L) and 37 (15 upregulated vs. 22 downregulated, $40.54 \%$ upregulation rate, for RC-H vs. RC-M) flavonoids were identified as differential metabolites, containing both biomarker and common differential metabolites (CDM). From the perspective of biomarker, the ratio of upregulation to downregulation in RC-H vs. RC-L is 2:0, and the upregulation rate was $100 \%$; the ratio is $2: 1$ for RC-M vs. RC-L, with an upregulation rate of $66.7 \%$; and the ratio is $1: 0$ for RC-H vs. RC-M, with an upregulation rate of $100 \%$. These results suggest that elevation in the range of 3933-4531 m drives an overall enhancement of flavonoid biosynthesis.

Based on their corresponding aglucones, 25 (RC-H vs. RC-L), 38 (RC-M vs. RC-L) and 26 (RC-H vs. RC-M) were further grouped into four subclasses: quercetin and derivatives 
$(12,2$ biomarkers + 10 CDMs, for RC-H vs. RC-L; 20, 3 biomarkers + 17 CDMs, for RC-M vs. RC-L; 9, 1 biomarker +8 CDMs, for RC-H vs. RC-M), kaempferol and derivatives (6 CDMs for RC-H vs. RC-L, 8 CDMs for RC-M vs. RC-L, and 7 CDMs for RC-H vs. RC-M), catechins and derivatives (7 CDMs for RC-H vs. RC-L, 6 CDMs for RC-M vs. RC-L, and 6 CDMs for RC-H vs. RC-M), and Luteolin and derivatives (0 CDM for RC-H vs. RC-L, 4 CDMs for RC-M vs. RC-L, and 4 CDMs for RC-H vs. RC-M). They accounted for $67.57 \%$ of $37,67.86 \%$ of 56 , and $70.27 \%$ of 37 of the corresponding flavonoid differential metabolites, respectively.

Some studies have shown that these four flavonoids are important representative flavonoid classes in Rhodiola crenulate [4]. Therefore, the changes of these four flavonoids with altitude will be further discussed in detail to elucidate the specific alteration characteristics of the major representative flavonoids in Rhodiola crenulata in response to altitude changes in the range of 3933-4531 m.

In RC-H vs. RC-L, 2 biomarkers and 10 CDMs were classified into the class of quercetin and its derivatives. According to the FC value size, these 12 substances are presented in Figure 7A in a descending order from left to right. We observed that 2 biomarkers and 8 CDMs were upregulated and 2 CDMs were downregulated for a total of 12 substances, suggesting that the elevation from 3933-4531 m overall enhanced the biosynthesis of quercetin and its derivatives class. Further analysis revealed that of these 12, 10 were quercetin glycosides (all showed upregulation with the exception of quercetin-3-O- $\left(2^{\prime \prime}-\mathrm{O}\right.$ glucosyl) glucuronide(Q3O(2OG) glucuronide)), and only two were aglucones, namely Dihydroquercetin (downregulated) and 7-O-Methxyl Quercetin (7OMQ, upregulated). This suggests that the increase in altitude from 3933-4531 m contributed to the enhanced biosynthesis of various quercetin glycosides, making the overall change more active, while the biosynthetic species of quercetin aglucones were less affected.

In RC-M vs. RC-L, 3 biomarkers and 17 CDMs were classified into the class of quercetin and its derivatives. These 20 substances are presented in Figure 7B in a descending order from left to right according to the FC value size. As seen in the Figure, 2 biomarkers and 15 CDMs were upregulated, and 2 CDMs and 1 biomarker were downregulated, which indicates that the elevation from 3933-4249 m generally enhanced the biosynthesis of quercetin and its derivatives class. Further analysis revealed that 17 were quercetin glycosides, except for quercetin-3-O-robinobioside (Q3ORo), quercetin-3-O-(2/"-O-glucosyl) glucuronide (Q3O(2OG) glucuronide) and quercetin-3-O-(6"'-galloyl) galactoside (Q3O(6G)G), which were downregulated, while the other 14 showed upregulation; only three were aglucones, all of which were upregulated, namely two methoxy aglucones (7-O-Methxyl Quercetin (7OMQ), 5-O-Methylquercetin (5OM)) and quercetin. This suggests that the increase in altitude from 3933-4249 m enhanced the biosynthesis of multiple quercetin glycosides, while the aglucone species were relatively less affected, which is similar to RC-H vs. RC-L.

In $\mathrm{RC}-\mathrm{H}$ vs. RC-M, one biomarker and eight CDMs were classified into the class of quercetin and its derivatives. According to the size of FC value, these 9 substances are presented in Figure $7 \mathrm{C}$ in descending order from left to right. We can see that one biomarker (quercetin-3-O-(6" -galloyl) galactoside (Q3O(6G)G), 7396.52-fold change) and one CDM (quercetin-3-O-robinobioside (Q3ORo), 3.03-fold change) were upregulated for 2 substances and downregulated for 7 CDMs (for comparison, their FC values were normalized so that their range is 2.22-14.37), indicating a weakening of the enhanced effect of elevation from $4249-4531 \mathrm{~m}$ on the biosynthesis of quercetin and its derivatives.

Based on the results of pairwise comparisons of Rhodiola crenulata at the above three altitudes (3933, 4249 and $4531 \mathrm{~m}$ ), we speculate that there may be a weak point affecting the biosynthesis of quercetin and its derivatives at an altitude of about $4200 \mathrm{~m}$. The increase from below this weak point to above it has a weak effect on the enhancement of the biosynthesis of quercetin and its derivatives in Rhodiola crenulata. 

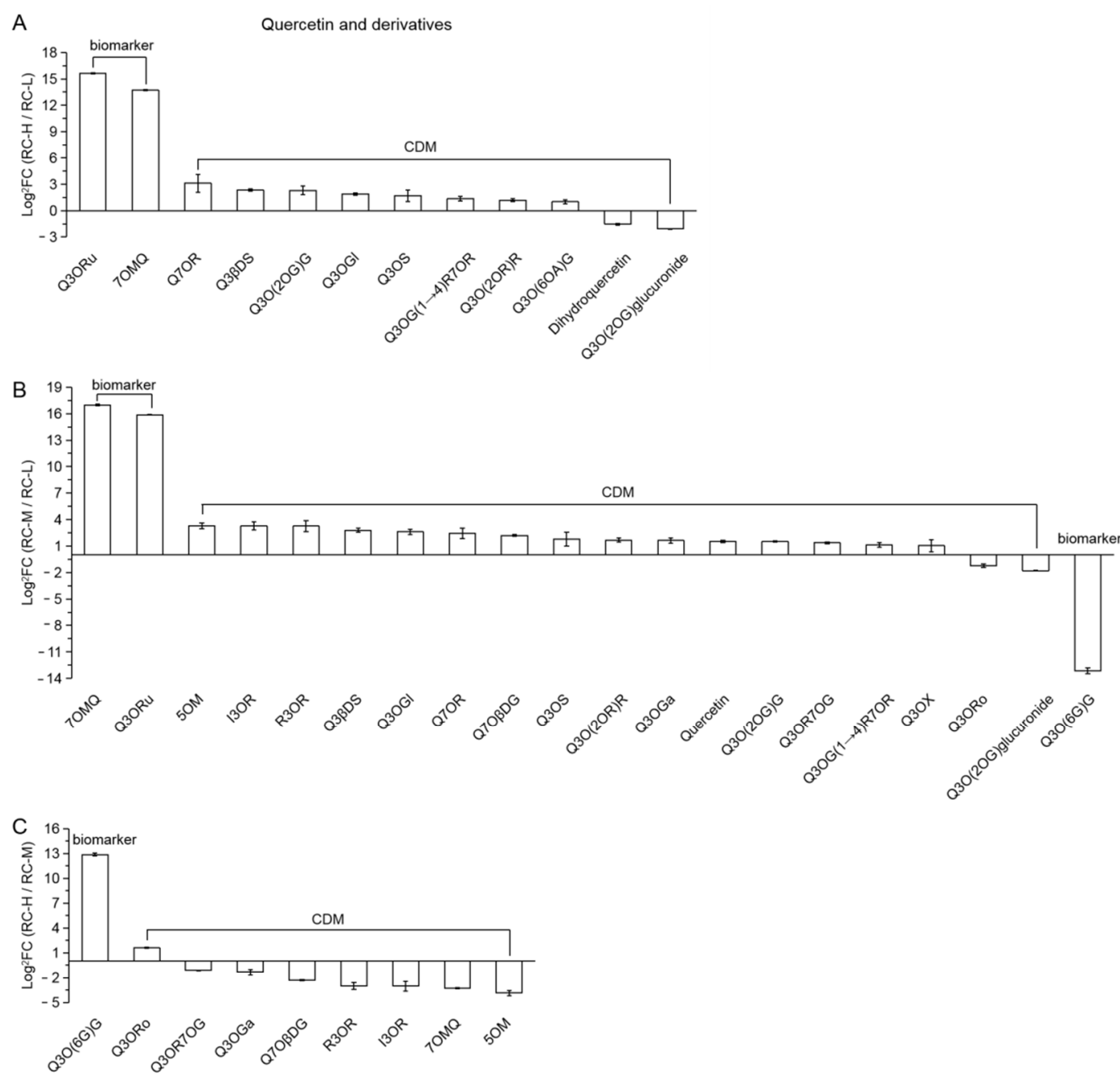

Figure 7. Comparison of differential metabolites of quercetin and its derivatives between RC-L, RC-M and RC-H. These were 2 biomarkers and 10 common differential metabolites (CDMs) for RC-H vs. RC-L (A), 3 biomarkers and 17 CDMs for RC-M vs. RC-L (B), and 1 biomarker and 8 CDMs for RC-H vs. RC-M (C), respectively. Upregulation denotes that the content of a metabolite in RC increased with elevation between two altitudes, and the opposite for downregulation. The $\mathrm{Y}$-axis is shown as $\mathrm{Log}^{2} \mathrm{FC}$ values for the convenience of visual presentation (Note: The up-amplitude in the text refers to the FC value). The $\mathrm{X}$-axis denotes metabolite names.

In summary, the above results clearly demonstrate that elevation increases contribute to more active biosynthesis of quercetin and its derivative classes between the altitude of 3933-4531 m, mainly through the upregulation of multiple glycosides. Meanwhile, the number of differential metabolites of quercetin and its derivative classes was 12, 20 and 9 in the comparison of RC-H vs. RC-L, RC-M vs. RC-L, and RC-H vs. RC-M, which reached $32.43 \%$ of $37,35.71 \%$ of 56 and $24.32 \%$ of 37 of the total number of flavonoid differential metabolites, respectively. This indicates that when the elevation change from 3933-4531 m, quercetin and its derivative classes accounted for the top of the significant variation in flavonoid metabolites in Rhodiola crenulata, amounting to about $1 / 3$ of the total flavonoid differential metabolites.

It was found that exposure of Vicia faba [80], Brassica napus [26] and apple fruits [81] to UV-B radiation resulted in a significant increase in quercetin biosynthesis. Quercetin-3O-rutinoside (Q3ORu, Rutin) and 7-O-Methxyl Quercetin (7OMQ, Rhamnetin) are both important antioxidants in plants $[82,83]$, and we found that these substances were significantly upregulated as biomarkers. Based on the above findings, we suggest that the 
upregulation of quercetin and its derivatives at middle and high altitudes may be closely related to enhancing Rhodiola crenulata's resistance to increased high-altitude UV stress.

Among RC-H vs. RC-L and RC-M vs. RC-L, 6 and 8 CDMs were classified into the class of kaempferol and its derivatives, which are presented in Figure 8A,B in descending order according to the FC value, respectively. We found that all CDMs were upregulated except for 8-Prenylkaempferol which showed downregulation in RC-M vs. RC-L on the right-most side. Incorporating the results in Figure 8C, the 7 CDMs with the 3 upregulated and 4 downregulated in RC-H vs. RC-M, the biosynthetic patterns of kaempferol and its derivatives in the altitude range from 3933-4531 $\mathrm{m}$ were similar to those of quercetin and its derivatives, but the difference was that kaempferol and its derivatives did not show biomarker for the differential metabolites in the altitude range from 3933-4531 m. This indicates that the response of kaempferol and its derivatives to the changes in this altitude range was slightly smaller than that of the former.
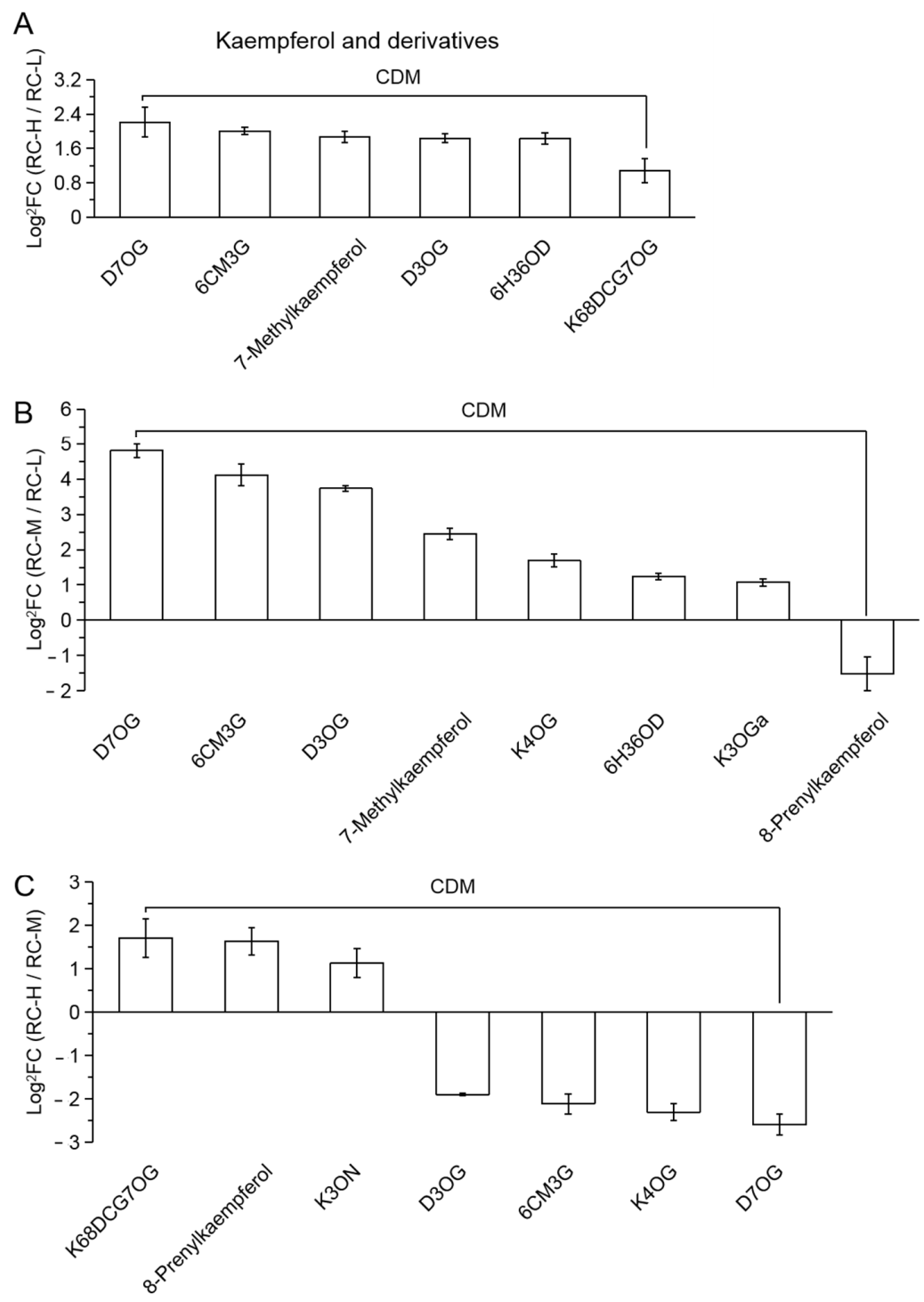

Figure 8. Comparison of differential metabolites of kaempferol and its derivatives between RC-L, RC-M and RC-H. These were 6 CDMs for RC-H vs. RC-L (A), 8 CDMs for RC-M vs. RC-L (B), and 7 CDMs for RC-H vs. RC-M (C), respectively. Upregulation denotes that the content of a metabolite in $\mathrm{RC}$ increased with elevation between two altitudes, and the opposite for downregulation. The Y-axis is shown as $\log ^{2} \mathrm{FC}$ values for the convenience of visual presentation (Note: The up-amplitude in the text refers to the FC value). The $\mathrm{X}$-axis denotes metabolite names. 
Meanwhile, the number of differential metabolites of kaempferol and its derivatives was 6, 8 and 7 in the comparisons of RC-H vs. RC-L, RC-M vs. RC-L, and RC-H vs. RC-M. Within the range of variation, kaempferol and its derivatives accounted for the second highest significant variation in flavonoid metabolites in Rhodiola crenulata with $16.22 \%$ of $37,14.29 \%$ of 56 and $18.92 \%$ of 37 in the total number of flavonoid differential metabolites, respectively, slightly lower than quercetin and its derivatives.

In RC-H vs. RC-L, seven CDMs were classified into the class of catechin and derivatives. These 7 substances are presented in Figure 9A in descending order from left to right according to the size of FC values. As seen in the figure, all seven CDMs were upregulated, indicating that the elevation from 3933-4531 m enhanced the biosynthesis of catechin and derivatives. Further analysis revealed that the leftmost five were condensed tannin (most significantly upregulated, with a range of FC values from 9.02-4.73), and then the catechin (3.92-fold change) and (-)-Epicatechin-3-(3"'-O-methyl) gallate ((-)-E3(3OM)G, 3.58-fold change). This suggests that the increase in elevation from $3933-4531 \mathrm{~m}$ contributed to the overall enhancement of catechin and its derivative-like biosynthesis, specifically in the form of enhanced condensed tannin biosynthesis.

In RC-H vs. RC-M, six CDMs were classified into catechin and derivatives. These six substances are presented in Figure $9 \mathrm{C}$ in a descending order from left to right according to the $\mathrm{FC}$ value. We can see that all six CDMs were upregulated (FC values ranged from 5.62-2.22), indicating that the elevation from 4249-4531 m enhanced the biosynthesis of catechin and derivatives, which was similar to RC-H vs. RC-L, i.e., the enhanced condensed tannin biosynthesis.

In RC-M vs. RC-L, six CDMs were classified into the catechin and derivatives. These six substances are presented in Figure $9 \mathrm{~B}$ in descending order from left to right according to the FC value. As seen in Figure 9B, four CDMs were upregulated (FC values ranging from 6.12-2.11, containing two condensed tannin) and two CDMs were downregulated (FC values ranging from $0.495-0.45$, containing one condensed tannin). This indicates that the elevation from 3933-4249 m generally enhanced the biosynthesis of catechin and derivatives represented by condensed tannin. However, compared to all upregulation in RC-H vs. RC-L and RC-H vs. RC-M, some catechin and derivative metabolites showed downregulation in RC-M vs. RC-L. This suggests that there may be influence on the altered biosynthesis of catechin and derivatives around $4000 \mathrm{~m}$.

Based on the above results of pairwise comparisons of catechin and derivatives at three altitudes (3933, 4249 and $4531 \mathrm{~m}$ ), we hypothesized that there might be an intensification point affecting the alteration of biosynthesis of catechin and derivatives in Rhodiola crenulata around $4000 \mathrm{~m}$, i.e., ascending from below $4000 \mathrm{~m}$ to above $4000 \mathrm{~m}$, the enhancement of catechins and their derivatives-like biosynthesis in Rhodiola crenulata starts to occur. This pattern was significantly strengthened from $4249-4531 \mathrm{~m}$ (i.e., RC-H vs. RC-M), all CDMs were upregulated, 6 upregulated vs. 0 downregulated. From 3933-4531 m (i.e., $\mathrm{RC}-\mathrm{H}$ vs. RC-L), the enhancement is most pronounced (all CDMs are upregulated and more numerous, 7 upregulated vs. 0 downregulated), i.e., it focuses on the enhanced development of condensed tannin biosynthesis.

Meanwhile, the number of catechin and its derivative-like differential metabolites was 7, 6 and 6 in the comparisons of RC-H vs. RC-L, RC-M vs. RC-L, and RC-H vs. RC-M, which account for $18.92 \%$ of $37,10.71 \%$ of 56 , and $16.22 \%$ of 37 in the total number of flavonoid differential metabolites, respectively. The catechin and derivatives accounted for the third most significant variation in flavonoid metabolites in Rhodiola crenulata within the elevation range variation. 

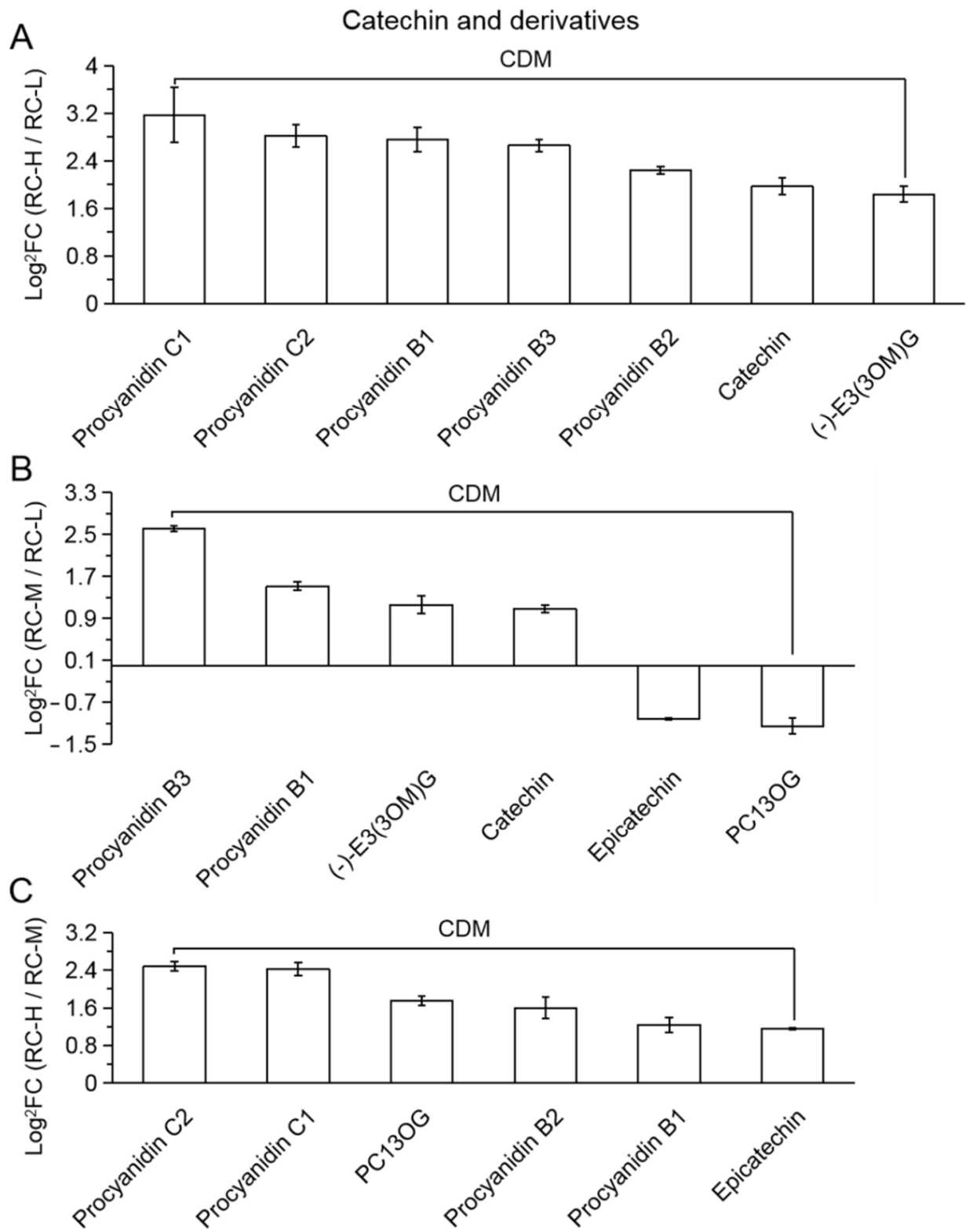

Figure 9. Comparison of differential metabolites of catechin and derivatives between RC-L, RC-M and RC-H. These were 7 CDMs for RC-H vs. RC-L (A), 6 CDMs for RC-M vs. RC-L (B), and 6 CDMs for RC-H vs. RC-M (C), respectively. Upregulation denotes that the content of a metabolite in RC increased with elevation between two altitudes, and the opposite for downregulation. The Y-axis is shown as $\log ^{2} \mathrm{FC}$ values for the convenience of visual presentation (Note: The up-amplitude in the text refers to the FC value). The $\mathrm{X}$-axis denotes metabolite names.

The pattern of variation of Luteolin and their derivative classes was less clear than the three aforementioned flavonoid classes, with only four CDMs presented in RC-M vs. RC-L and RC-H vs. RC-M, respectively, while no differential metabolites were presented in RC-H vs. RC-L. From Figure 10A,B, although there were 2 upregulated and 2 downregulated CDMs in both RC-M vs. RC-L and RC-H vs. RC-M, however, in RC-M vs. RC-L, it exhibited 2 upregulated as glycosides (luteolin-3'-O-glucoside (L3OG); luteolin-7-O-(2" O-rhamnosyl)-O-rhamnosyl) rutinoside (L7O(2OR)R)), 2 downregulated to aglucones (Luteolin and Isoluteolin), and the pattern reversed in RC-H vs. RC-M, i.e., 2 upregulated to aglucones (Luteolin and Isoluteolin), 2 downregulated to glycosides (Luteolin-7-Oglucoside (Cynaroside) and luteolin-3'-O-glucoside (L3OG)). Thus, it indicates that the 
biosynthesis of Luteolin and its derivatives showed little response to elevation changes in the range of 3933-4531 $\mathrm{m}$ and did not show significant patterns.
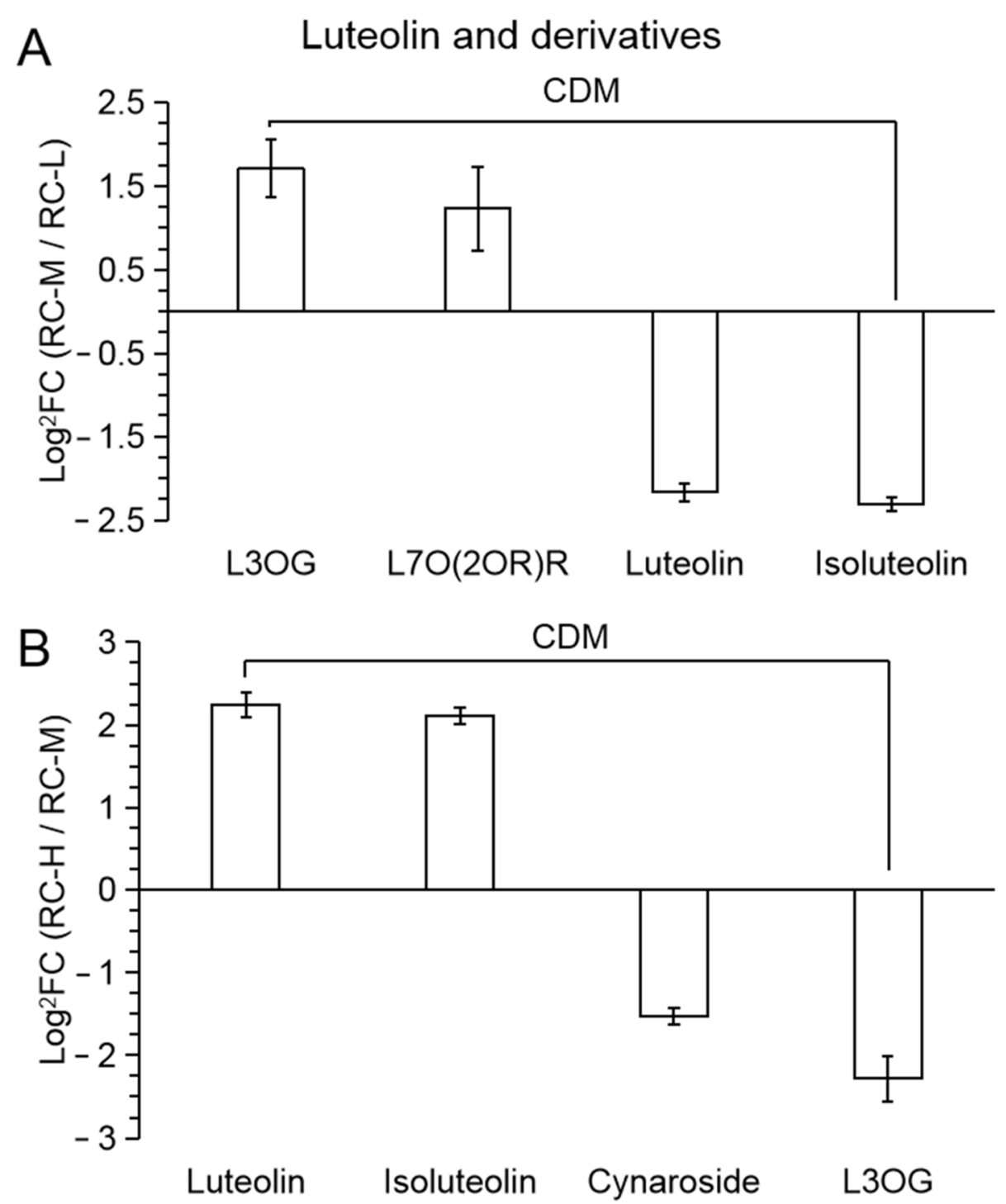

Figure 10. Comparison of differential metabolites of luteolin and its derivatives between RC-L, RC-M and RC-H. These were 0 CDMs for RC-H vs. RC-L, 4 CDMs for RC-M vs. RC-L (A), and 4 CDMs for RC-H vs. RC-M (B), respectively. Upregulation denotes that the content of a metabolite in RC increased with elevation between two altitudes, and the opposite for downregulation. The Y-axis is shown as $\log ^{2} \mathrm{FC}$ values for the convenience of visual presentation (Note: The up-amplitude in the text refers to the $\mathrm{FC}$ value). The $\mathrm{X}$-axis denotes metabolite names.

The number of differential metabolites of Luteolin and its derivative classes was 0,4 and 4 in the comparisons of RC-H vs. RC-L, RC-M vs. RC-L and RC-H vs. RC-M. It reaches $0 \%$ of $37,7.14 \%$ of 56 and $10.81 \%$ of 37 of the total number of differential metabolites of flavonoids, respectively, which indicates that Luteolin and its derivative classes were not significantly affected by the above elevation changes due to the share of flavonoid metabolites in Rhodiola crenulata was also small.

By analyzing the specific change patterns of the above four major representative flavonoids of Rhodiola crenulata with altitude changes, it can be seen that the altitude increase in the range of 3933-4531 m contributed to the more active biosynthesis of the flavonoids of Rhodiola crenulata, which is characterized by: (1) the enhanced synthesis was evident in quercetin, catechin and kaempferol and their derivatives, with the most 
significant upregulation in quercetins, followed by kaempferols and then catechins, while the changes in luteolins were less clear; (2) there may be an intensification point affecting the biosynthesis of catechin and derivatives at around $4000 \mathrm{~m}$, and there may be a weakening point affecting the biosynthesis of flavonoids represented by quercetin and its derivatives and kaempferol and its derivatives at around $4200 \mathrm{~m}$; (3) the enhancement of biosynthesis of quercetin and kaempferol and their derivatives by the effect of altitude was mainly in the form of their glycosides, while the biosynthesis of catechin and derivatives was mainly characterized by the synthesis of condensed tannins.

This enhanced biosynthesis of flavonoids, mainly in the form of flavonoid glycosides, with increasing altitude generally agrees with our previous studies [14].

The new findings of this study include (1) the flavonoid catechin and derivatives exhibited mainly focused synthesis of condensed tannins with elevation in the range of 3933-4531 m, and (2) the enhanced biosynthesis of flavonoid quercetin and its derivatives was particularly significant in response to elevation in the range of 3933-4531 m.

The above results support that the total flavonoids and total phenols contents increased significantly with altitude (as shown in Figure 3A,B), and further reveal that the enhanced biosynthesis of quercetin, kaempferol, and catechin and their derivatives were the main contributors, among which the contribution of quercetin and its derivatives may be particularly important.

When combined with the results of Figure 3D, which showed a significant increase in condensed tannins from 3933-4531 m, these results suggest that the enhanced biosynthesis of catechin and derivatives is the main contributor, with condensed tannins being particularly important in terms of quantity and magnitude of upregulation.

Some studies have reported an enhanced effect of UV light or low temperature on the biosynthesis of quercetins, condensed tannins and flavonoid glycosides in plants $[81,84,85]$. These substances have a variety of resilient biological activities against UV radiation, low temperature or drought $[21,25,26]$, and our results are consistent with these previous findings. There are inflection points in the literature showing the effect of altitude on different flavonoids, which corroborates our results [86-88].

Considering the previous literatures, we suggest that flavonoid glycosides represented by quercetins, and catechins represented by condensed tannins, both contribute to the altitudinal challenge of Rhodiola crenulata, conferring a strong adaptive capacity to altitudeintegrated adversities consisting of UV, low temperature, hypoxia, and oxidative stress.

Quercetin, kaempferol, and catechins play important roles in cancer prevention or treatment of COVID-19 [89-91]. The flavonoid glycoside contributes to the bioavailability of flavonoid substances to the body, and condensed tannins play an important role in promoting human health [92-95]. Combining the existing works, we suggest that Rhodiola crenulata grown at higher altitudes may have more advantages for the supplementation of flavonoids of quercetin, kaempferol, and catechins represented by condensed tannins and for improving the bioavailability of these flavonoids for human health.

\section{Gallic Acid and derivatives}

Among RC-H vs. RC-L and RC-M vs. RC-L, 9 CDMs and 12 (1 biomarker + 11 CDMs) differential metabolites were classified in this category, including gallic acid derivatives and hydrolyzed tannins formed from gallic acid and sugar, respectively. They are presented in a descending order from left to right in Figure 11A,B, respectively, based on the size of FC values. We found that the number of downregulations was significantly higher than the number of upregulations ( 6 downregulations vs. 3 upregulations; 8 downregulations vs. 4 upregulations), indicating that the elevation from $3933-4531 \mathrm{~m}$ and from $3933-4249 \mathrm{~m}$ both significantly attenuated the biosynthesis of gallic acid and its derivatives. We further analyzed the downregulated metabolites from the chemical structure perspective, and found that for the 6 downregulated CDMs in RC-H vs. RC-L, four were hydrolyzed tannins except digallic acid and ellagic acid; 8 downregulations were found in RC-M vs. RC-L (7 CDMs + 1 biomarker at the rightmost), all 7 belonged to the hydrolyzed tannins except ethyl gallate, which included 1,6-di-O-galloyl-D-glucose (16DOGDG) as a biomarker 
(0.00001-fold change). These results indicate that hydrolyzed tannins exhibit significant downregulation with increasing altitude, i.e., from 3933-4531 m and from 3933-4249 m.
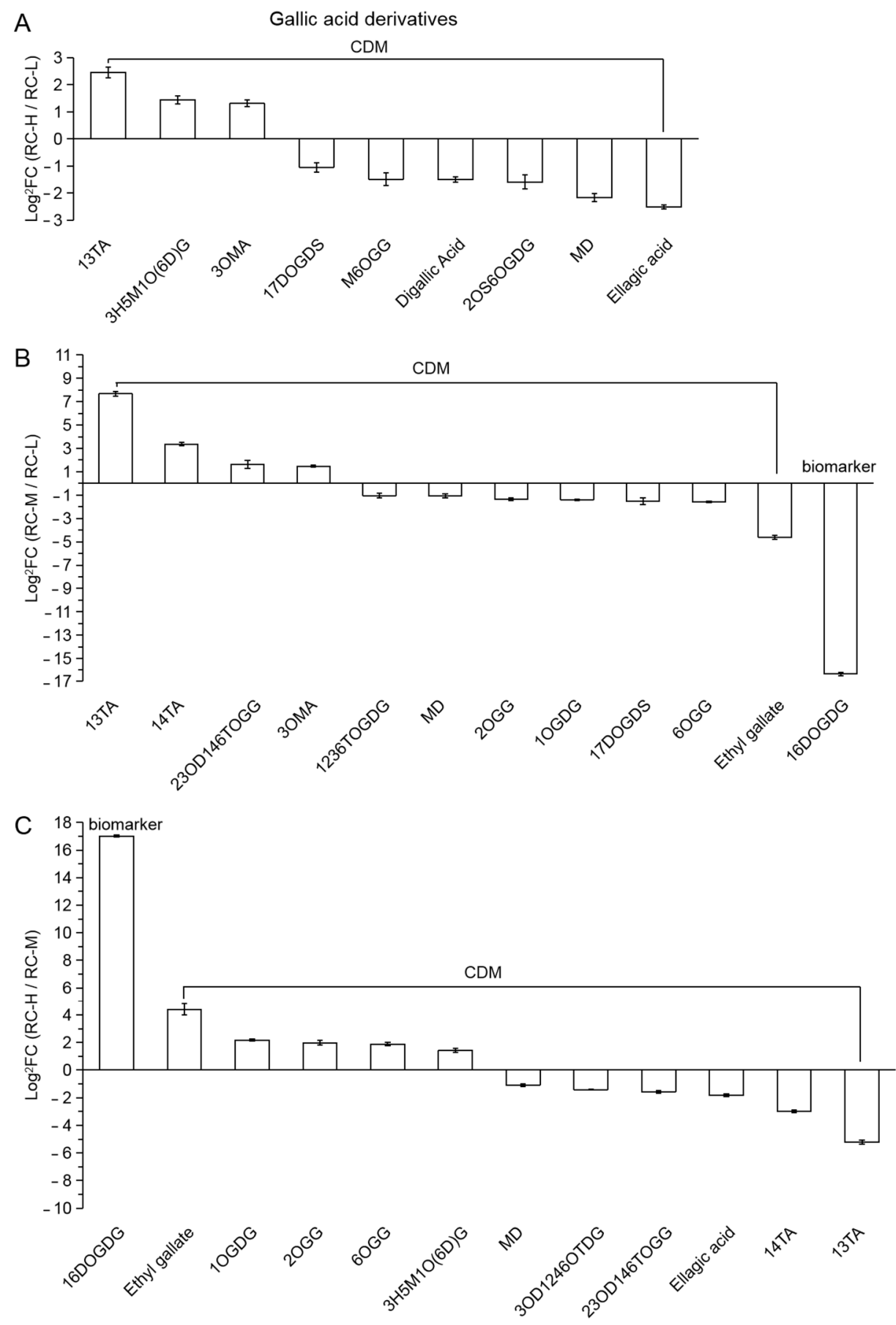

Figure 11. Comparison of differential metabolites of gallic acid derivatives between RC-L, RC-M and RC-H. These were 9 CDMs for RC-H vs. RC-L (A), 1 biomarker and 11 CDMs for RC-M vs. RC-L (B), and 1 biomarker and 11 CDMs for RC-H vs. RC-M (C). Upregulation denotes that the content of a metabolite in RC increased with elevation between two altitudes, and the opposite for downregulation. The $\mathrm{Y}$-axis is shown as $\log ^{2} \mathrm{FC}$ values for the convenience of visual presentation (Note: The up-amplitude in the text refers to the FC value). The $\mathrm{X}$-axis denotes metabolite names.

As shown in Figure 11C, the 1biomarker and 11CDMs in RC-H vs. RC-M were classified in this category, and their variation characteristics were partially different from 
RC-H vs. RC-L and RC-M vs. RC-L. As seen in Figure 11C, the number of downregulated is the same as the number of upregulated (6 downregulated vs. 6 upregulated), and the number of their downregulated hydrolyzed tannins versus upregulated counterparts is 3 downregulated (Monogalloyl-diglucose; 3-O-Digalloyl-1,2,4,6-O-tetragalloyl-D-glucose; 2,3-O-Digalloyl-1,4,6-tri-O-galloyl- glucose) vs. 4 upregulated (1,6-Di-O-Galloyl-D-Glucose as biomarker; 1-O-Galloyl-D- glucose; 2-O-Galloyl-glucose; 6-O-Galloyl-glucose). This indicates that the increase in altitude from $4244-4531 \mathrm{~m}$ had little effect on the biosynthesis of gallic acid derivatives.

Based on the results of the pairwise comparisons of Rhodiola crenulata at the above three altitudes (3933, 4249 and $4531 \mathrm{~m}$ ), we speculate that there may be an intensification point affecting the biosynthesis of gallic acid derivatives and hydrolyzed tannins in Rhodiola crenulata at about $4200 \mathrm{~m}$, and the increase from below this intensification point to above it has an reverse effect on the weakening of the biosynthesis of gallic acid and its derivatives in Rhodiola crenulata.

In summary, the above results indicate that elevation in the range of 3933-4531 m generally weakens the biosynthesis of gallic acid and its derivatives mainly by promoting the downregulation of various hydrolyzed tannins. This result is generally consistent with our previous finding [14].

Combined with previous studies [96-99], we suggest that the downregulation of gallic acid and its derivatives in Rhodiola crenulata rhizomes with elevation may be related to changes in biotic stress and UV intensity changes with elevation.

Considering the significant upregulation of condensed tannins in catechin and derivatives with altitude, our finding of an overall downregulation of hydrolyzed tannins with altitude explains the increase in total tannins content (total tannins = condensed tannins + hydrolyzed tannins) from 3933-4531 m, which is significantly weaker than the increase in condensed tannins content (Figure 3C,D).

\section{Phenylpropanoids}

Among the 237 phenylpropanoid metabolites characterized, 47 (28 upregulation vs. 19 downregulation), 48 (26 upregulation vs. 22 downregulation) and 44 ( 26 upregulation vs. 18 downregulation) phenylpropanoids were identified as differential metabolites in $\mathrm{RC}-\mathrm{H}$ vs. RC-L, RC-M vs. RC-L and RC-H vs. RC-M, containing biomarker and CDM, respectively. These results indicated a overall upregulation of phenylpropanoids in response to elevation in the range of 3933-4531 m.

Based on their basic structures, 47 (RC-H vs. RC-L), 48 (RC-M vs. RC-L) and 44 (RC-H vs. RC-M) were further grouped into four subclasses. These four subclasses, namely Cinnamic acid-Coumaroyl and derivatives, phenylmethanes, phenylethanes and shikimic acids.

Therefore, the changes of these four phenylpropanoids with altitude will be further discussed in detail to elucidate the specific patterns of changes of phenylpropanoids in Rhodiola crenulata with altitude.

Twenty-four ( 6 biomarkers and $18 \mathrm{CDMs}$ ) and 23 (9 biomarkers and $14 \mathrm{CDMs}$ ) differential metabolites in RC-H vs. RC-L and RC-H vs. RC-M were classified as Cinnamic acid-Coumaroyl and its derivatives, respectively. They are presented in a descending order according to the FC values from left to right in Figure 12A,C, respectively. We observe that the number of upregulations is significantly higher than the number of downregulations (15 upregulations vs. 9 downregulations; 15 upregulations vs. 8 downregulations). Six biomarkers in RC-H vs. RC-L show significant upregulations, with FC values ranging from 25,852.22-147.26. Seven biomarkers out of nine in RC-H vs. RC-M display significant upregulations, with a FC from 1654.26-147.26. Two biomarkers showed a significant downregulation (0.0003- and 0.0002-fold change, respectively). These results suggest that the biosynthesis of Cinnamic acid-Coumaroyl and its derivatives was significantly enhanced by increasing altitude from $3933-4531 \mathrm{~m}$ and from $4249-4531 \mathrm{~m}$. 
A
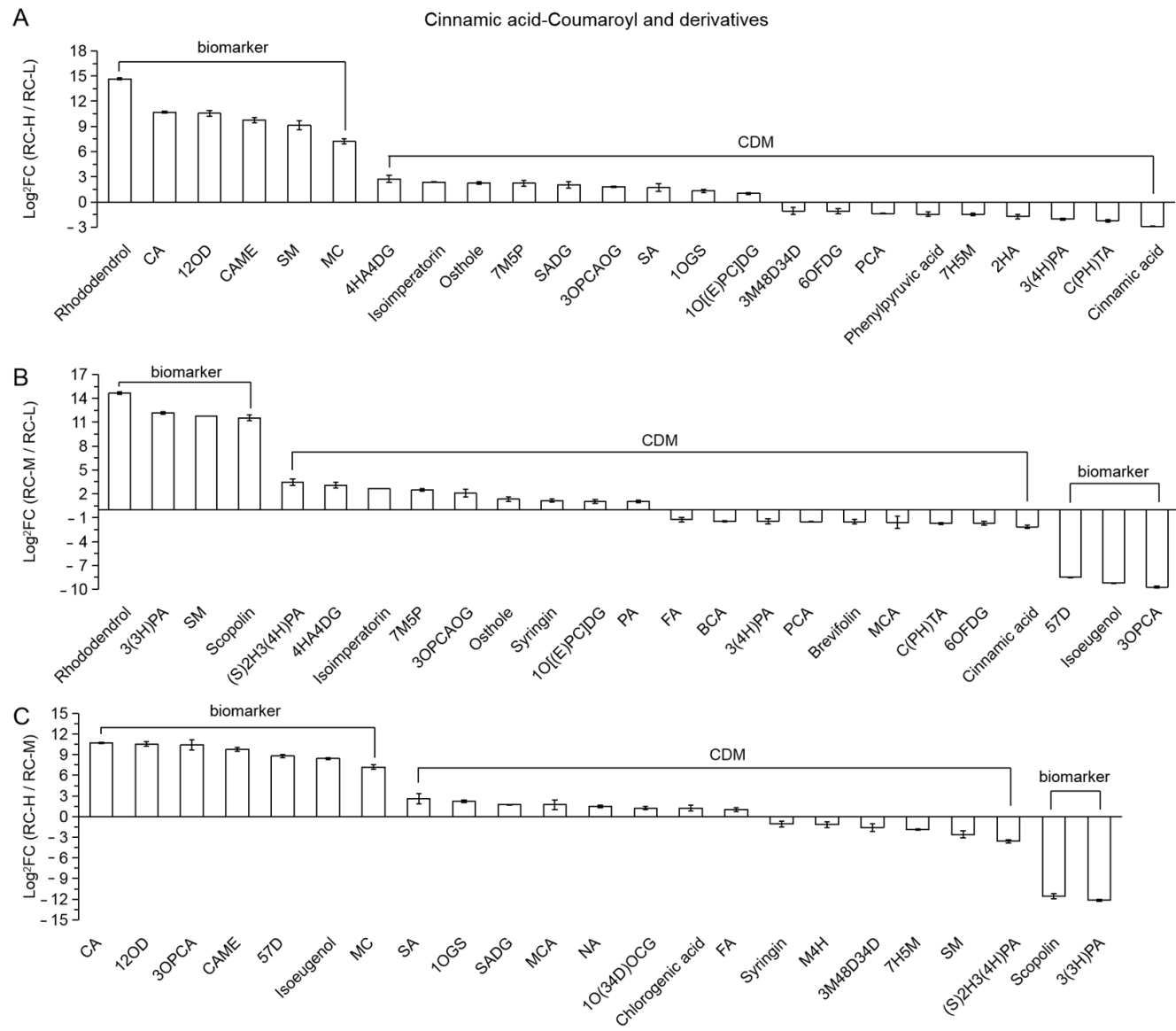

Figure 12. Comparison of differential metabolites of Cinnamic acid-Coumaroyl and its derivatives between RC-L, RC-M and RC-H. These were 6 biomarkers and 18 CDMs for RC-H vs. RC-L (A), 7 biomarkers and 18 CDMs for RC-M vs. RC-L (B), and 9 biomarkers and 14 CDMs for RC-H vs. RC-M (C). Upregulation denotes that the content of a metabolite in RC increased with elevation between two altitudes, and the opposite for downregulation. The Y-axis is shown as $\log ^{2} \mathrm{FC}$ values for the convenience of visual presentation (Note: The up-amplitude in the text refers to the FC value). The $\mathrm{X}$-axis denotes metabolite names.

Further analysis from the perspective of chemical structure revealed that 11 of the 15 upregulations in RC-H vs. RC-L were metabolites of phenylpropanes indirectly derived from Cinnamic acid-Coumaroyl via the phenylpropanoids pathway (Cinnamic acid-Coumaroyl indirect derivatives), such as Caffeoylbenzoyltartaric acid, 1,2-O-Diferuloylglycerol and Chlorogenic acid methyl ester. This includes all 6 upregulated biomarkers out of 11 . The other 4 upregulated ones are direct derivatives of Cinnamic acid-Coumaroyl, which are 4-Hydroxycinnamyl alcohol 4-D-glucoside (4HA4DG), 7-methoxy-5-prenyloxycoumarin (7M5P), 3-O-p-Coumaroylquinic acid O-glucoside (3OPCAOG) and 1-O-[(E)-p-coumaroyl]D-glucose (1O[(E)PC]DG); 13 out of the 15 upregulations in RC-H vs. RC-M are indirect Cinnamic acid-Coumaroyl derivatives, which includes five upregulated biomarkers, and the remaining two belongs to direct derivatives of Cinnamic acid-Coumaroyl, i.e., 3-O-pCoumaroylquinic acid (3OPCA) and 5,7-dimethoxycoumarin (57D). The number of indirect derivatives vs. direct derivatives regarding the 9 downregulated CDMs in RC-H vs. RC-L was 4 vs. 5; the number of indirect derivatives vs. direct derivatives regarding the 8 downregulated (6 CDMs +2 biomarkers at the rightmost) in RC-H vs. RC-M was 5 (including 2 biomarkers) vs. 3 .

Token together, the number of indirect derivatives vs. direct derivatives was 15 vs. 9 in RC-H vs. RC-L, and 18 vs. 5 in RC-H vs. RC-M, and the number of upregulations is significantly higher than the number of downregulations for the indirect derivatives (11 
upregulations vs. 4 downregulations in RC-H vs. RC-L; 13 upregulations vs. 5 downregulations), while changes of direct derivatives were less clear (also See Tables S2 and S4). These results indicate that with increasing altitude (i.e., from 3933-4531 $\mathrm{m}$ and from 4249-4531 $\mathrm{m})$, the biosynthesis of the indirect Cinnamic acid-Coumaroyl-derived phenylpropane metabolites via the phenylpropane pathway is more affected than the direct Cinnamic acid-Coumaroyl derivatives, and being obviously upregulated.

Seven biomarkers and 18 CDMs in RC-M vs. RC-L were classified into this category, including indirect and direct derivatives. As shown in Figure 12B, the number of upregulated is slightly higher than the number of downregulated (13 upregulated vs. 12 downregulated), however, from the biomarker perspective, the upregulated biomarkers are higher than the downregulated biomarkers (4 vs. 3), with FC values of 25,319.26-2996.11 vs. $0.003-0.001$. The number of indirect derivatives vs. direct derivatives was 17 vs. 8 , and the number of upregulations is slightly higher than the number of downregulations for the indirect derivatives ( 9 upregulations vs. 8 downregulations (also See Table S3). Above findings revealed that the enhancing trend of biosynthesis of Cinnamic acid-Coumaroyl and its derivative classes, especially for the indirect derivatives (i.e., phenylpropanes) starts to appear as the altitude increased from 3933-4249 m.

Based on the results of the above-mentioned pairwise comparisons of Rhodiola crenulata at three altitudes (3933, 4249 and $4531 \mathrm{~m}$ ), it was observed that the tendency of enhanced biosynthesis of Cinnamic acid-Coumaroyl and its derivatives from 3933-4249 m started to appear, and was mainly characterized by the response of indirect derivatives (i.e., phenylpropanes), which was more evident during the increase from $3933-4531 \mathrm{~m}$ and from 4249-4531 m.

Based on the above results, we found for the first time that the change of phenylpropanoids with elevation was characterized by the enhanced synthesis of metabolites indirectly derived from Cinnamic acid-Coumaroyl via the phenylpropanoids pathway (hereafter referred to as "phenylpropanoids variation characteristics"). Also, phenylpropanes highlighted these characteristics here.

In addition, the number of Cinnamic acid-Coumaroyl and its derivative-like differential metabolites was 24,25 and 23 in the comparisons of RC-H vs. RC-L, RC-M vs. RC-L, and $\mathrm{RC}-\mathrm{H}$ vs. RC-M, reaching $51.06 \%$ of $47,52.08 \%$ of 48 and $52.27 \%$ of 44 of the total differential metabolites of phenylpropanoids, respectively. This indicates that the Cinnamic acid-Coumaroyl and its derivatives accounted for the most significant differential variation of phenylpropanoids in the elevation range change from 3933-4531 m.

Among RC-H vs. RC-L and RC-H vs. RC-M, 15 (2 biomarkers and 13 CDMs) and 10 CDMs differential metabolites were classified as phenylmethanes, respectively. They are presented in a descending order from left to right in Figure 13A,C according to the size of FC values. As can be seen from the figure, the number of upregulations is significantly higher than the number of downregulations (10 upregulations vs. 5 downregulations; 8 upregulations vs. 2 downregulations), and two and one upregulated biomarkers existed in $\mathrm{RC}-\mathrm{H}$ vs. $\mathrm{RC}-\mathrm{L}$ and $\mathrm{RC}-\mathrm{H}$ vs. RC-M, respectively. These results indicate that the elevation from 3933-4531 m and from 4249-4531 m significantly enhances the biosynthesis of phenylmethanes. 


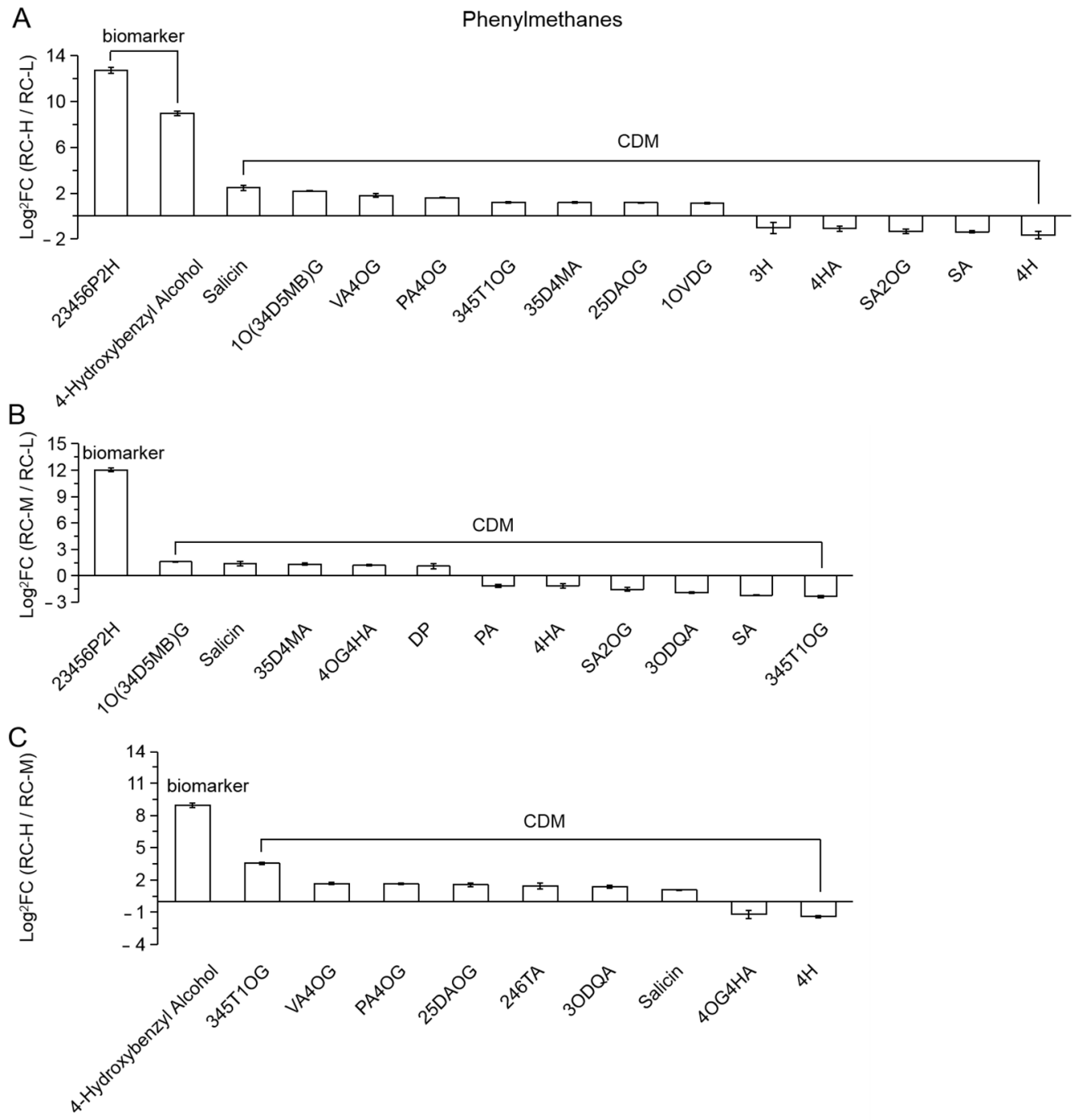

Figure 13. Comparison of differential metabolites of phenylmethanes between RC-L, RC-M and RC-H. These were 2 biomarkers and 13 CDMs for RC-H vs. RC-L (A), 1 biomarker and 11 CDMs for RC-M vs. RC-L (B), and 10 CDMs for RC-H vs. RC-M (C). Upregulation denotes that the content of a metabolite in RC increased with elevation between two altitudes, and the opposite for downregulation. The Y-axis is shown as $\log ^{2} \mathrm{FC}$ values for the convenience of visual presentation (Note: The up-amplitude in the text refers to the FC value). The $\mathrm{X}$-axis denotes metabolite names.

One biomarker and 11 CDMs were classified in RC-M vs. RM-L. As seen in Figure 13B, the number of upregulated was equal to the number of downregulated ( 6 upregulated vs. 6 downregulated), however; while from the perspective of biomarker, the upregulated was significantly higher than the downregulated (1 vs. 0), i.e., 2,3,4,5,6-pentahydroxyhexyl 2-hydroxybenzoate, with an FC value of 4217.04. It can be concluded that the biosynthesis of phenylmethanes is enhanced by the increase in altitude from 3933-4249 m. Combining the results of the above three altitudes $(3933,4249$ and $4531 \mathrm{~m})$, we found that the response of phenylmethanes was consistent with that of Cinnamic acid-Coumaroyl and its derivatives with altitude, i.e., the trend of enhanced biosynthesis of phenylmethanes began to appear from 3933-4249 $\mathrm{m}$. This tendency was more obvious during the elevation from 3933-4531 $\mathrm{m}$ and from 4249-4531 $\mathrm{m}$. It should be noted that phenylmethanes can still be considered as a class of metabolites indirectly derived from Cinnamic acid-Coumaroyl via the phenylpropanoids pathway, thus it further enriches the above-mentioned "phenylpropanoids variation characteristics". 
In addition, the number of differential metabolites of phenylmethanes was 15, 12 and 10 in the comparisons of RC-H vs. RC-L, RC-M vs. RC-L, and RC-H vs. RC-M, which reached $31.92 \%$ of $47,25 \%$ of 48 and $22.73 \%$ of 44 of the total differential metabolites of phenylpropanoids, respectively. This indicates that the phenylmethanes class is immediately behind Cinnamic acid-Coumaroyl and its derivatives for significant differences in the metabolites of phenylpropanoids over the elevation range of 3933-4531 m.

The number of differential metabolites of phenylethanes in RC-H vs. RC-L, RC$\mathrm{M}$ vs. RC-L and RC-H vs. RC-M were 0 vs. 5, 3 vs. 4 and 3 vs. 6, respectively, as shown in Figure 14A-C, showing an overall trend of downregulation of phenylethanes with elevation. This indicates that medium and low altitudes are more beneficial for the accumulation of phenylethanes in Rhodiola crenulata rhizomes compared to high altitudes. In particular, it should be noted that phenylethanes can still be considered as the category of metabolites indirectly derived from Cinnamic acid-Coumaroyl via the phenylpropanoids pathway. Therefore, from the perspective of "phenylpropanoids variation characteristics", the elevation enhancement for the phenylpropanoids metabolites indirectly derived from Cinnamic acid-Coumaroyl via the phenylpropanoids pathway is more specific for the phenylpropanes and phenylmethanes, while the trend is reversed for phenylethanes. It should be also recalled here that the direct Cinnamic acid-Coumaroyl derivatives is less affected.
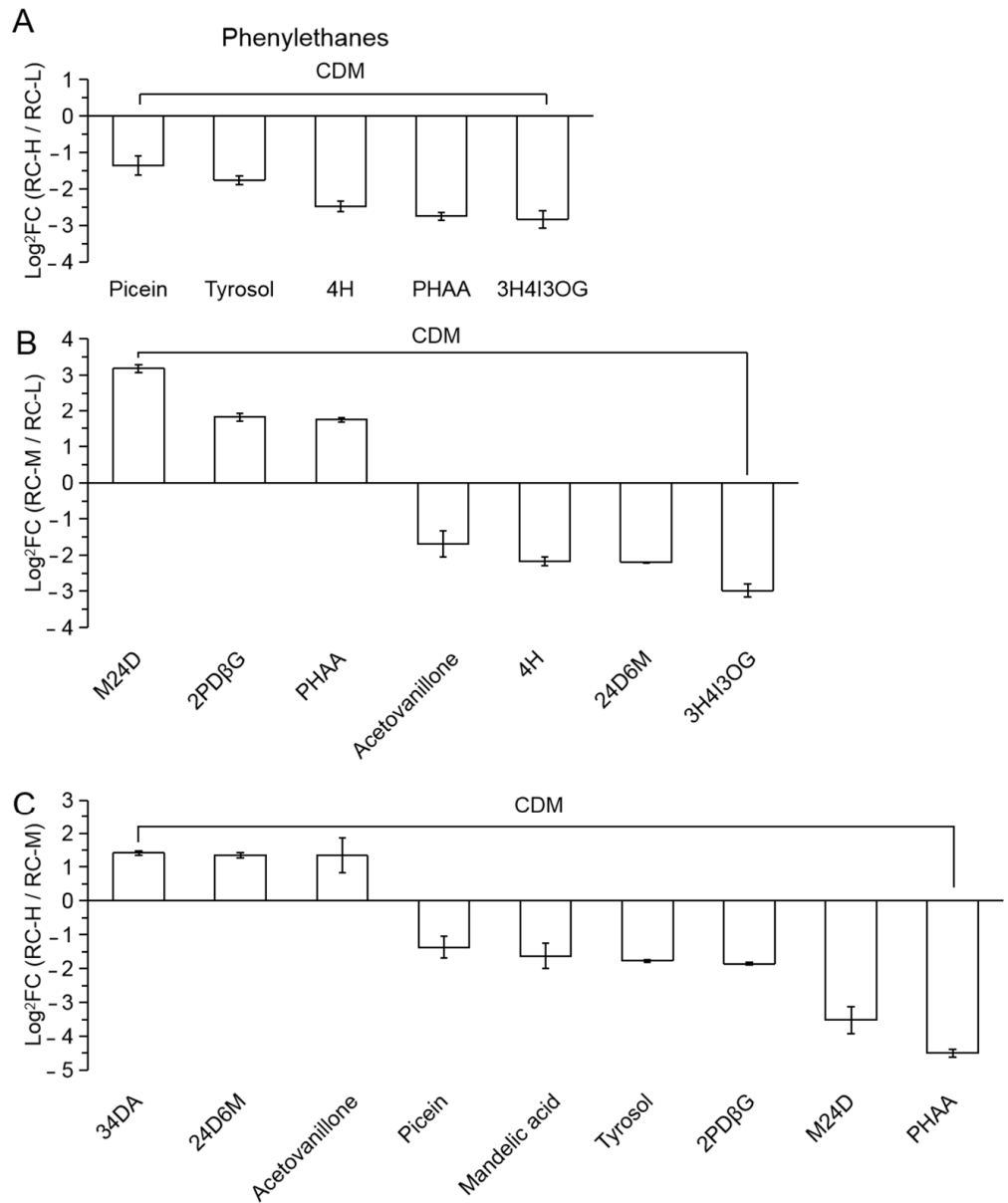

Figure 14. Comparison of differential metabolites of phenylethanes between RC-L, RC-M and RC-H. These were 5 CDMs for RC-H vs. RC-L (A), 7 CDMs for RC-M vs. RC-L (B), and 9 CDMs for RC-H vs. RC-M (C). Upregulation denotes that the content of a metabolite in RC increased with elevation between two altitudes, and the opposite for downregulation. The Y-axis is shown as $\log ^{2} \mathrm{FC}$ values for the convenience of visual presentation (Note: The up-amplitude in the text refers to the FC value). The X-axis denotes metabolite names. 
The number of phenylethanes differential metabolites was 5, 7 and 9 in RC-H vs. RC-L, RC-M vs. RC-L and RC-H vs. RC-M, reaching $10.64 \%$ of $47,14.58 \%$ of 48 and $20.46 \%$ of 44 of the total number of phenylpropanoids differential metabolites, respectively. This indicates that in the range of 3933-4531 m, phenylethanes category was inferior to Cinnamic acid-Coumaroyl and derivatives and phenylmethanes. Therefore, it can be concluded that the effect of elevation increase in the range of 3933-4531 m on the biosynthesis of phenylpropanoids is still dominated by an enhanced tone. Combined with the quantitative perspective of biomarkers (recall that regarding upregulated biomarkers in RC-H vs. RC-L, RC-M vs. RC-L, and RC-H vs. RC-M, 9, 7 and 8 belong to phenylpropanoids while 2, 2 and 1 were flavonoids), the upregulated biomarkers of phenylpropanoids were found to be numerically more dominant in the elevation range of 3933-4531 m, suggesting that the enhancement of phenylpropanoids biosynthesis should be more dominant than flavonoids in this elevation range.

It is reported that phenylpropanoids enhance plant resistance to UV radiation, low temperature, drought, and pathogen [22-24], and it also has antioxidant, anti-inflammatory, and anti-diabetic effects as well as treating neuroinflammation-related diseases [100-102]. Combining these findings, we suggest that Rhodiola crenulata, grown at higher altitudes, may be of greater advantage for the beneficial effects of phenylpropanoids supplementation on human health.

In the comparisons of RC-H vs. RC-L, RC-M vs. RC-L and RC-H vs. RC-M, the number of upregulated and downregulated differential metabolites of shikimic acids were 3 vs. 0,4 vs. 0 and 0 vs. 2, respectively (Figure 15A-C). In terms of biomarkers, there were one upregulated biomarker in RC-H vs. RC-L with FC value of 3020.26 , and two upregulated biomarkers in RC-M vs. RC-L with FCs of 60,226.67 and 9381.78, respectively. These results indicate that shikimic acids show a significant upregulation with altitude, but it should be noted that RC-H vs. RC-M showed the opposite situation, which does not fully coincide with the overall upregulation.

Shikimic acid-phenylalanine is an upstream metabolic pathway of phenylpropanoidsflavonoids metabolism $[35,36]$. Our results for the overall upregulation of shikimic acids with increasing altitude in the altitude range of 3933-4531 m support the finding that the phenylpropanoids-flavonoids biosynthesis were enhanced with increasing altitude. Meanwhile, the results of downregulation of shikimic acids in RC-H vs. RC-M coincide with the changes of quercetin and kaempferol and their derivatives in RC-H vs. RC-M. This suggests that the weakening trend of quercetins and kaempferols biosynthesis in elevation from $4244-4531 \mathrm{~m}$ may be related to the downregulation of shikimic acids in RC-H vs. RC-M.

We argue that the enhanced upregulation of the shikimic acid-phenylpropanoidsflavonoids metabolic pathway is highly correlated with the overall intensification of stresses from UV radiation, low temperature, and hypoxia due to elevation.

\section{Amino Acids}

The number of upregulated and downregulated amino acid and derivative-like differential metabolites in the comparisons of RC-H vs. RC-L, RC-M vs. RC-L and RC-H vs. RC-M were 39 vs. $4 ; 17$ vs. 13 and 39 vs. 10, respectively (Figure 16A-C). In terms of biomarkers, there are four and three upregulated biomarkers, respectively; while in $\mathrm{RC}-\mathrm{H}$ vs. RC-M, there is one upregulation and one downregulation. These results suggest that amino acid and derivative classes show a significant upregulation with increase of elevation. 

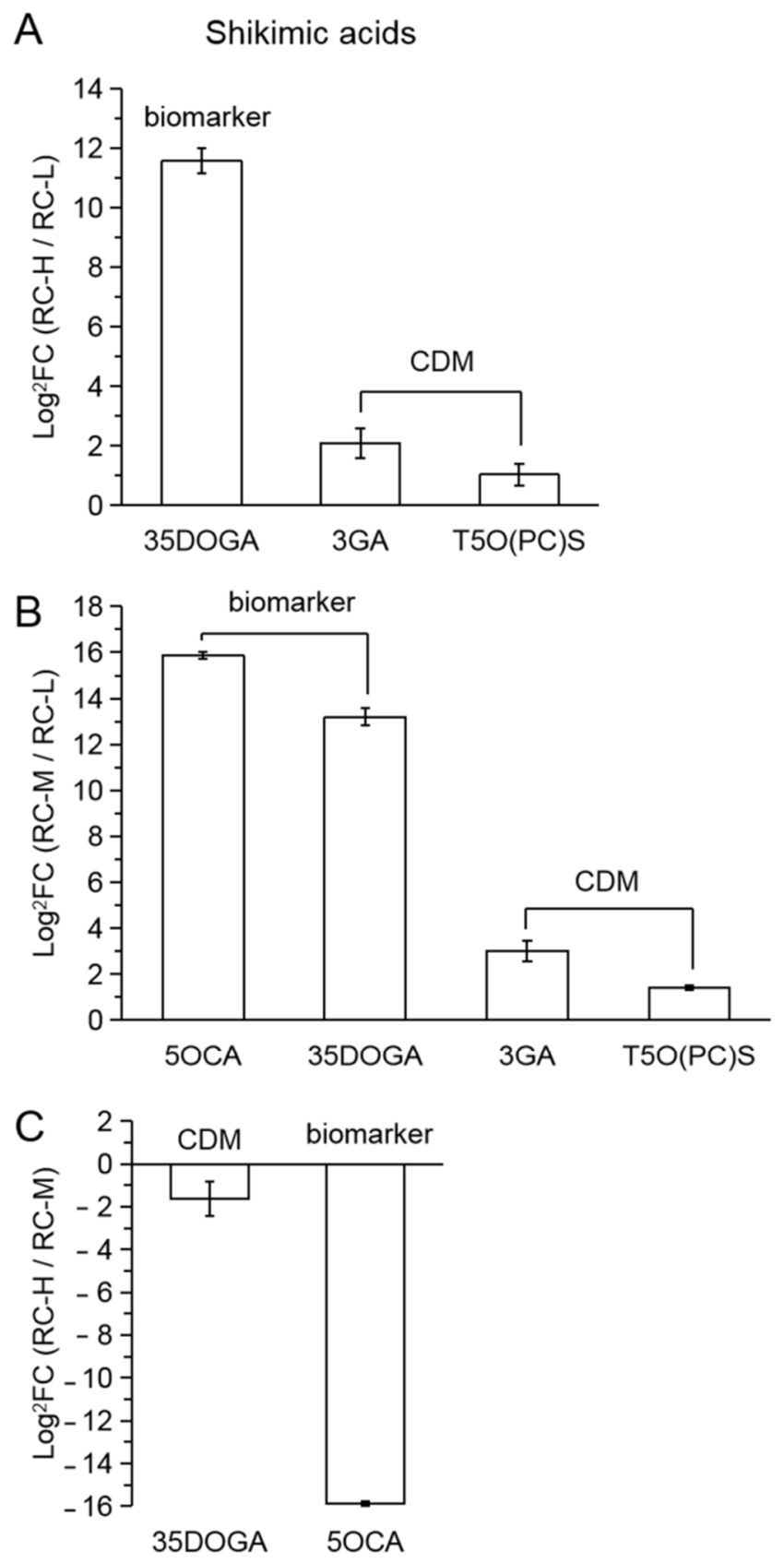

Figure 15. Comparison of differential metabolites of shikimic acids between RC-L, RC-M and RC-H. These were 1 biomarker and 2 CDMs for RC-H vs. RC-L (A), 2 biomarkers and 2 CDMs for RC-M vs. RC-L (B), and 1 biomarker and 1 CDM for RC-H vs. RC-M (C). Upregulation denotes that the content of a metabolite in RC increased with elevation between two altitudes, and the opposite for downregulation. The $\mathrm{Y}$-axis is shown as $\log ^{2} \mathrm{FC}$ values for the convenience of visual presentation (Note: The up-amplitude in the text refers to the FC value). The $\mathrm{X}$-axis denotes metabolite names. 

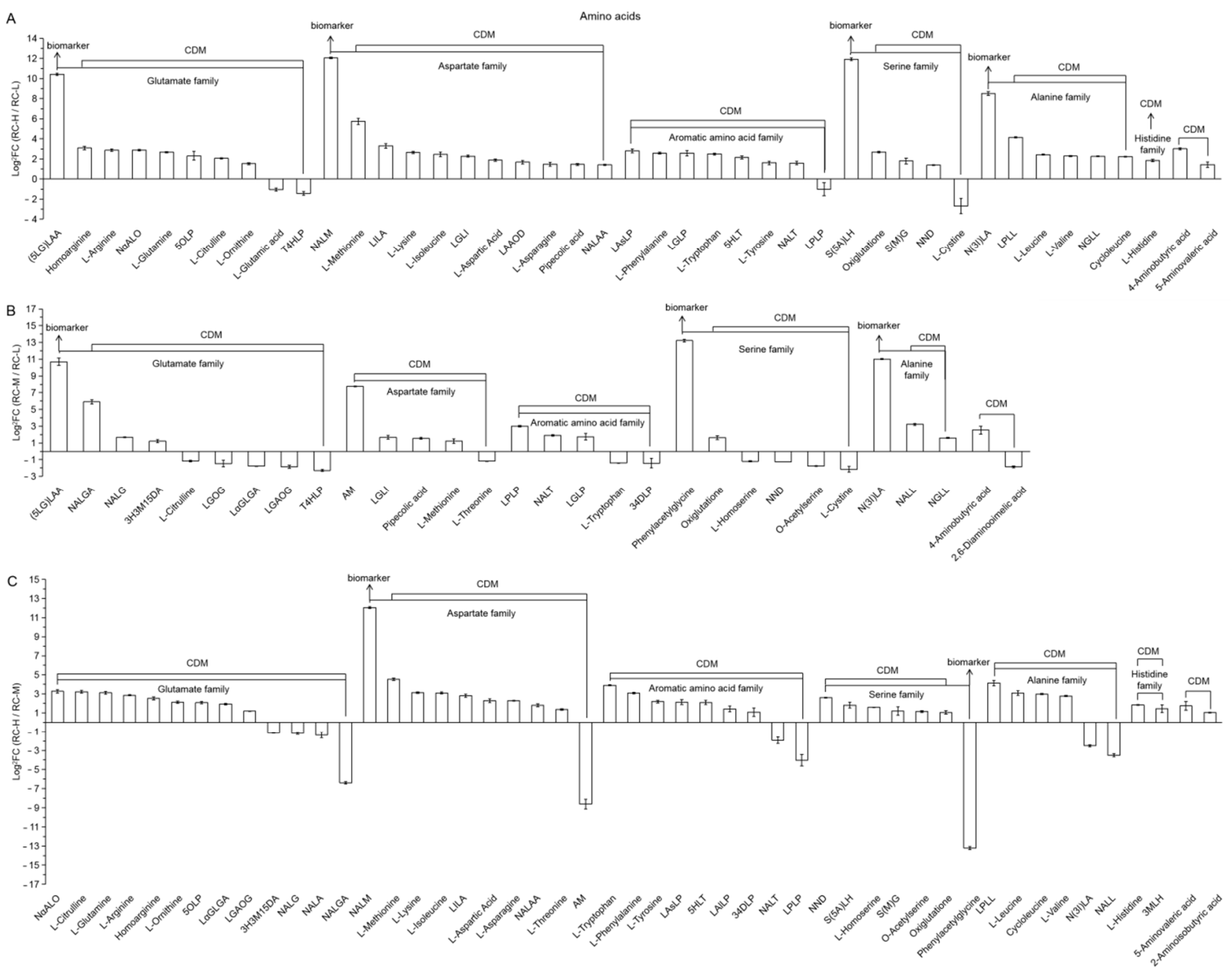

Figure 16. Comparison of differential metabolites of amino acids between RC-L, RC-M and RC-H. These were 4 biomarkers and 39 CDMs for RC-H vs. RC-L (A), 3 biomarkers and 27 CDMs for RC-M vs. RC-L (B), and 2 biomarkers and 47 CDMs for RC-H vs. RC-M (C). Upregulation denotes that the content of a metabolite in RC increased with elevation between two altitudes, and the opposite for downregulation. The Y-axis is shown as $\log ^{2} \mathrm{FC}$ values for the convenience of visual presentation (Note: The up-amplitude in the text refers to the FC value). The X-axis denotes metabolite names.

In terms of the extent to which the six families of amino acid were affected by elevation, the relationship between the number of differential metabolites per family (RC-H vs. RC-L, RC-M vs. RC-L, and RC-H vs. RC-M) and the total number of differential metabolites of amino acids is: glutamic acid family $(23.26-30 \%)>$ aspartic acid family $(16.67-25.58 \%)>$ aromatic family $(16.67-18.61 \%)>$ serine family $(11.63-20 \%)>$ alanine family $(10-13.95 \%)>$ histidine family $(0-4.08 \%)$.

For the glutamate family, glutamate and glutamine were its signature amino acids. The glutamate family shows 3 ( 2 upregulated (i.e., (5-L-Glutamyl)-L-amino acid as biomarker and L-Glutamine) and 1 downregulated for L-Glutamic acid), 6 (3 upregulated (i.e., (5-L-Glutamyl)-L-amino acid as biomarker, $\mathrm{N}$-Acetyl-L-glutamic acid and N-Acetyl-LGlutamine) and 3 downregulated (i.e., L-Glutamine-O-glycoside, L- $\alpha$-Glutamyl-L-Glutamic Acid and L-Glutamic acid-O-glycoside)) and 5 (3 upregulated (i.e., L-Glutamine, L- $\alpha$ Glutamyl-L-Glutamic Acid and L-Glutamic acid-O-glycoside) and 2 downregulated (i.e., $\mathrm{N}$-Acetyl-L-Glutamine and N-Acetyl-L-glutamic acid)) in RC-H vs. RC-L, RC-M vs. RC-L and RC-H vs. RC-M, respectively, accounting for $6.98 \%$ of $43,20.00 \%$ of 30 and $10.20 \%$ of 49 of the total number of amino acid-like differential metabolites. They are transamination pools for the synthesis of many other amino acids in plants $[103,104]$. Our results found a 
more active metabolism with altitude. This result favourably supports the finding that the amino acid biosynthetic pathway is enhanced with increase of elevation.

The phenylalanines in the aromatic family are important intermediate metabolites in the shikimic acid-phenylalanine-phenylpropanoids-flavonoids biosynthetic pathway. We found their number of differential metabolites in the comparisons of RC-H vs. RC-L, RC-M vs. RC-L and RC-H vs. RC-M to be 4 (3 upregulated vs. 1 downregulated, $75 \%$ upregulated, i.e., L-Aspartyl-L-Phenylalanine, L-Phenylalanine and L-Glycyl-L-phenylalanine to upregulation; L-Prolyl-L-Phenylalanine to downregulation), 3 (2 upregulated vs. 1 downregulated, $66.67 \%$ upregulated, i.e., L-Prolyl-L-Phenylalanine and L-Glycyl-L-phenylalanine to upregulation; 3,4-Dihydroxy-L-phenylalanine to downregulation) and 5 (4 upregulated vs. 1 downregulated, $80 \%$ upregulated, i.e., L-Phenylalanine, L-Aspartyl-L-Phenylalanine, L-Alanyl-L-Phenylalanine and 3,4-Dihydroxy-L-phenylalanine to upregulation; L-Prolyl-LPhenylalanine to downregulation), respectively.

It accounts for $9.30 \%$ of $43,10.00 \%$ of 30 and $10.20 \%$ of 49 of the total differential metabolites of amino acids, respectively. This indicates that it is more active in amino acid biosynthesis in response to altitude change, and its response to altitude change shows significant upregulation in terms of upregulation ratio. This finding, combined with the aforementioned results for shikimic acids, phenylpropanoids and flavonoids in response to altitude change, clearly outlines the complete metabolic pathway for the enhanced biosynthesis of shikimic acid-phenylalanine-phenylpropanoids-flavonoids biosynthesis pathway with increasing altitude, suggesting that Rhodiola crenulata synthesizes various phenylpropanoids and flavonoids through this pathway to systematically enhance the resistance to altitude stress.

Furthermore, among the six amino acid families with differential amino acids changing with altitude, various amino acids such as glutathione, tyrosine, arginine, cysteine, proline, ornithine, glycine, aspartate, lysine, isoleucine, and leucine enhance plant stress tolerance in multiple ways [105-115]; while valine and tryptophan are associated with promoting plant growth [116,117]. These differential amino acid changes suggest that Rhodiola crenulata may involve multiple forms of adaptive changes with altered growth patterns in response to altitude changes.

It is also important to note that 4-Aminobutyric acid was upregulated in $\mathrm{RC}-\mathrm{H}$ vs. RC-L (8.04-fold change) and RC-M vs. RC-L (5.75-fold change), while 2-Aminoisobutyric acid showed upregulation in RC-H vs. RC-M (2.07-fold change). This indicates that Aminobutyric acid is upregulated with elevation. It was found that aminobutyric acid is strongly associated with enhanced plant energy metabolism [34] and multiple stresses and defense systems [118,119].

\section{Free fatty acids and glycerides}

In RC-H vs. RC-L and RC-M vs. RC-L, 20 (1 biomarker and 19 CDMs) and 29 ( 3 biomarkers and $26 \mathrm{CDMs}$ ) differential metabolites were classified into this category, respectively. They are presented in Figure 17A,B in descending order from left to right on the basis of FC value size. As shown in the figure, the numbers of up- and downregulation were 13 vs. 7 and 24 vs. 5, respectively, indicating that the elevation increase from 3933-4531 m and from 3933-4249 m significantly enhanced the biosynthesis of free fatty acids and glycerides. 
A

Free fatty acids and glycerides
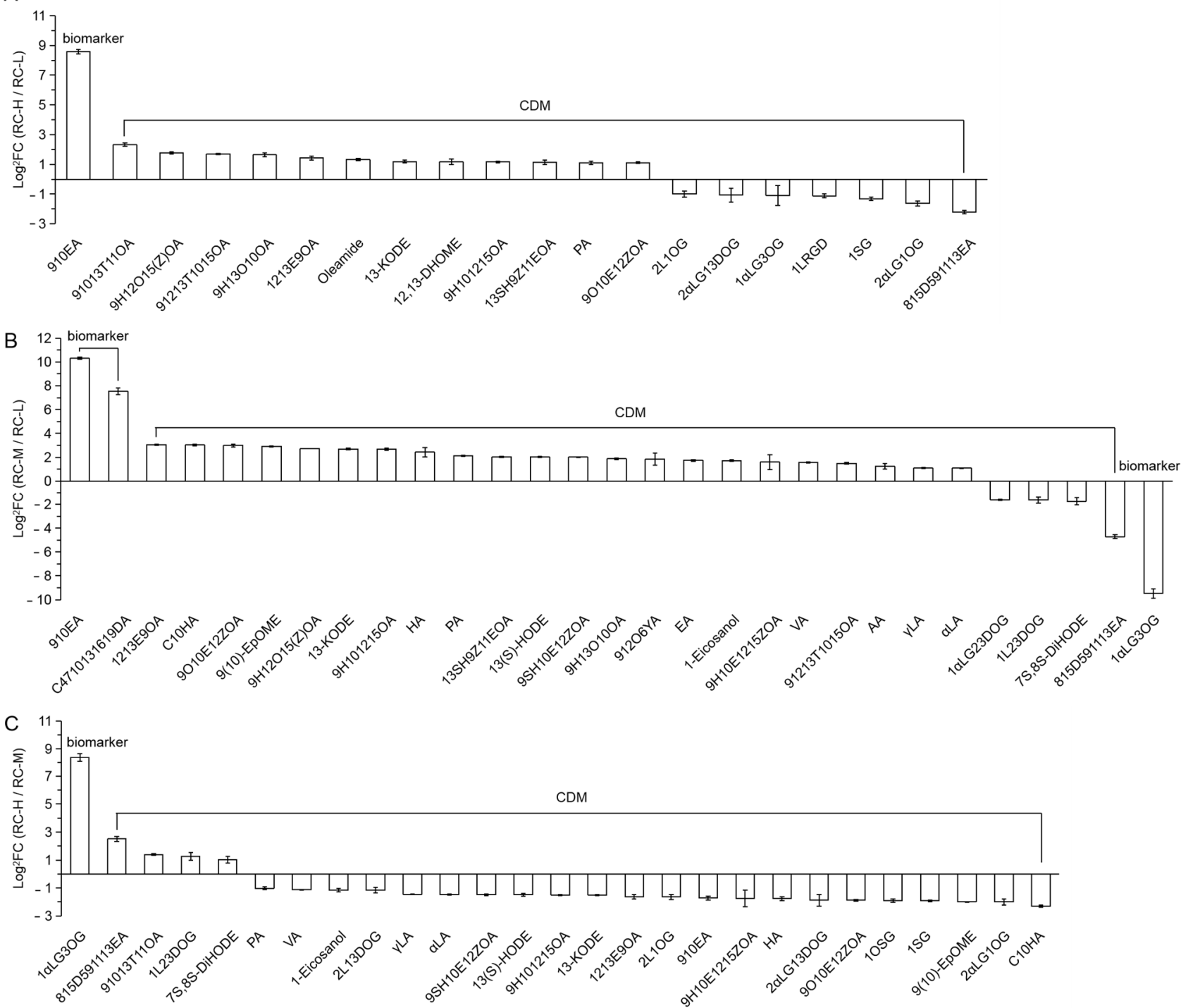

Figure 17. Comparison of differential metabolites of Free fatty acids and glycerides between RC-L, RC-M and RC-H. These were 1 biomarker and 19 CDMs for RC-H vs. RC-L (A), 3 biomarkers and 26 CDMs for RC-M vs. RC-L (B), and 1 biomarker and 26 CDMs for RC-H vs. RC-M (C). Upregulation denotes that the content of a metabolite in RC increased with elevation between two altitudes, and the opposite for downregulation. The Y-axis is shown as $\log ^{2} \mathrm{FC}$ values for the convenience of visual presentation (Note: The up-amplitude in the text refers to the FC value). The X-axis denotes metabolite names.

Further analysis from the chemical structure perspective revealed that all 13 upregulated CDMs in RC-H vs. RC-L were composed of free fatty acids, while the number of free fatty acids and glycerides was 1 vs. 6 in the 7 downregulated ones; among the 24 upregulated differential metabolites in RC-M vs. RC-L, the number of free fatty acids and glycerides is 24 vs. 0; while the number of free fatty acids and glycerides in the 5 downregulations was 2 vs. 3 . These results suggest that, when the elevation is increased from 3933-4531 m and from 3933-4249 m, free fatty acids were significantly upregulated, while glycerides were significantly downregulated. This indicates that the changes in free fatty acids and glycerides with altitude gradient in Rhodiola crenulata may be based on the breakdown of glycerides and their conversion into free fatty acids. It is important to note that this pattern was not evident in RC-H vs. RC-M, i.e., elevation from 4249-4531 m.

Kuczyńska et al. found an increase in free fatty acid content and a significant decrease in triglyceride content in barley (Hordeum vulgare L.) treated with abiotic stresses such as salt stress [120]. This result is in agreement with our findings. Glycerides are the major 
forms of lipid storage in organisms, while free fatty acids serve as the main form of lipid use that provides an essential energy source for the life activities of plants [55,56,121].

In the plant body, lipases are responsible for the breakdown of triglycerides to glycerol and fatty acids [122]. Therefore, it can be concluded that Rhodiola crenulata provides more source of energy to resist high altitude adversity stress by breaking down glycerides and accumulating free fatty acids at higher altitudes, thus improving the resistibility against high altitude integrated adversity.

\section{Nucleotides}

Among RC-H vs. RC-L, RC-M vs. RC-L and RC-H vs. RC-M, 19 CDMs, 25 (2 biomarkers and $23 \mathrm{CDMs}$ ) and 32 (2 biomarkers and $30 \mathrm{CDMs})$ differential metabolites are classified in this category, respectively. They are presented in a descending order from left to right in Figure 18A-C according to the size of FC values. As can be seen from the figure, the number of upregulated and downregulated is 12 vs. 7,10 vs. 15 and 19 vs. 13, respectively. This indicates that nucleotide biosynthesis was significantly enhanced when the altitude increased from $3933-4531 \mathrm{~m}$ and from $4249-4531 \mathrm{~m}$, while it was attenuated when the altitude increased from $3933-4249 \mathrm{~m}$. Considering the changes in altitude from 3933-4531 m, we concluded that the altitude increase in general contributed to the enhanced nucleotide synthesis in Rhodiola crenulata rhizome.

From a further analysis of the chemical structure, we found that the number ratio of up- and downregulated adenosine phosphates involved in ATP energy metabolism was 3 vs. 0, respectively ( 3 upregulated for Adenosine $5^{\prime}$-monophosphate, Adenosine $5^{\prime}$-diphosphate and Uridine $5^{\prime}$-monophosphate, respectively) for RC-H vs. RC-L; 2 vs. 0 (2 upregulated to NADP and Adenosine $5^{\prime}$-monophosphate) for RC-M vs. RC-L and 2 vs. 1 (2 upregulated to Adenosine $5^{\prime}$-monophosphate and Adenosine $5^{\prime}$-diphosphate, and 1 downregulated to NADP) for RC-H vs. RC-M. When converted to a percentage of nucleotide up- and downregulated differential metabolites, it was $25 \mathrm{vs.} \%$ for RC-H vs. RC-L; 20 vs. $0 \%$ for RC-M vs. RC-L; 10.53 vs. $7.69 \%$ for RC-H vs. RC-M (Figure 18A-C). These results indicate that adenosine phosphates exhibit upregulation as the elevation increase ranging from 3933-4531 m. In addition, adenosine triphosphate (ATP) was also upregulated in RC-H vs. RC-L (1.37-fold change) and RC-M vs. RC-L (2.60-fold change).

It was shown that Adenosine $5^{\prime}$-monophosphate, Adenosine $5^{\prime}$-diphosphate, Uridine $5^{\prime}$-monophosphate and ATP are directly related to energy metabolism [57,123].

Based on these findings, we suggest that elevation in the range of 3933-4531 m may enhance the adaptive capacity of Rhodiola crenulata against high altitude adversity by (1) upregulating nucleotide biosynthesis to promote more vigorous metabolism of genetic material and (2) supplying more abundant energy by upregulating adenosine phosphates, which is involved in ATP energy metabolism.

It should also be noted that, in conjunction with the findings of Figure 17, elevated altitude induced the upregulation of free fatty acids and downregulation of glycerides, thus providing energy to resist high altitude adversity stress. These findings provide a new insight into the resistance of Rhodiola crenulata to high altitude integrated adversity from an energetic perspective. 

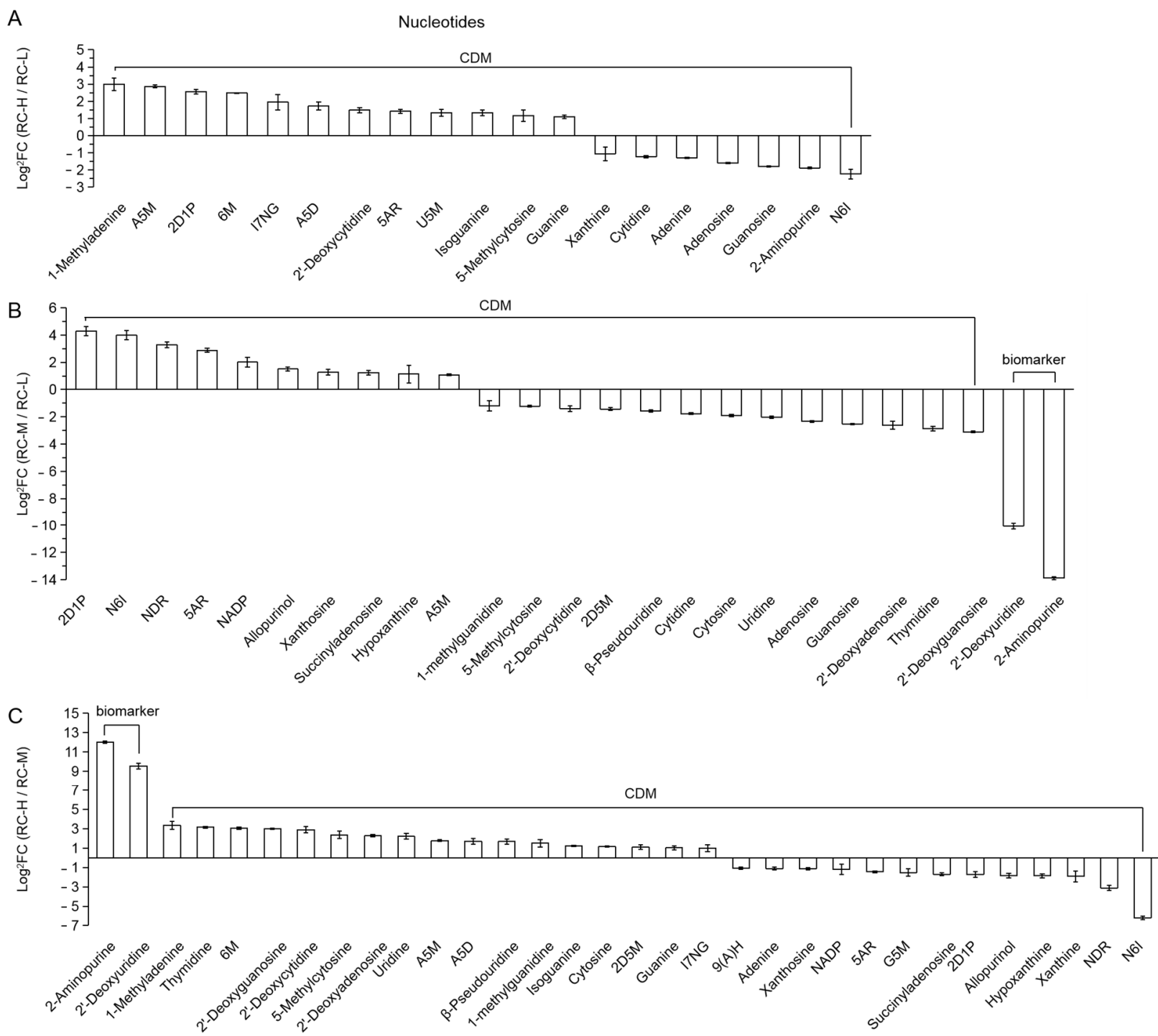

Figure 18. Comparison of differential metabolites of nucleotides between RC-L, RC-M and RC-H. These were 19 CDMs for RC-H vs. RC-L (A), 2 biomarkers and 23 CDMs for RC-M vs. RC-L (B), and 2 biomarkers and 30 CDMs for RC-H vs. RC-M (C). Upregulation denotes that the content of a metabolite in RC increased with elevation between two altitudes, and the opposite for downregulation The Y-axis is shown as $\log ^{2} \mathrm{FC}$ values for the convenience of visual presentation (Note: The up-amplitude in the text refers to the FC value). The $\mathrm{X}$-axis denotes metabolite names.

\section{Materials and Methods}

\subsection{Plant Materials and Extracts Preparation}

All Rhodiola crenulata samples were obtained from Dakazi Mountain, Ganzi Tibetan Autonomous Region, Sichuan Province, China, in October of 2019. According to altitudes,

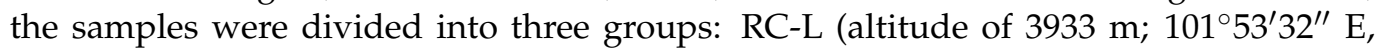
$28^{\circ} 56^{\prime} 4^{\prime \prime} \mathrm{N}$ ), RC-M (altitude of $4249 \mathrm{~m} ; 101^{\circ} 52^{\prime} 47^{\prime \prime} \mathrm{E}, 28^{\circ} 55^{\prime} 10^{\prime \prime} \mathrm{N}$ ) and RC-H (altitude of $4531 \mathrm{~m} ; 101^{\circ} 53^{\prime} 3^{\prime \prime}$ E, $28^{\circ} 55^{\prime} 22^{\prime \prime} \mathrm{N}$ ).

The collection sites could be characterized as a plateau type monsoon area with a alpine meadow soil. The average temperature of collection sites ranging from low to high altitude is $0.8--4.6^{\circ} \mathrm{C}$. The temperature would decrease by $0.90^{\circ} \mathrm{C}$ as elevation increased per $100 \mathrm{~m}$. No absolute frost-free period exists throughout the year with a precipitation $636 \mathrm{~mm} / \mathrm{y}$ and average annual sunshine of 1900-2600 $\mathrm{h}$ on the region. The annual light radiation is about $120-160 \mathrm{KCal} / \mathrm{cm}^{2}$. The UV radiation intensity at the collection sites at noon was 1558 (3933 m), 1857 (4249 m), 2486 (4531 m) uw/ $\mathrm{cm}^{2}$. 
For each group, three wild-grown Rhodiola crenulata plants were collected. All plants were authenticated as Rhodiola crenulata (Hook.f. \& Thomson) H.Ohba by Dr. Shubin Dong at the Beijing Forestry University, and were freeze-dried and stored at $-40{ }^{\circ} \mathrm{C}$.

For each group, the same weights were taken from rhizomes of three plants, then ground and mixed uniformly as one rhodiola sample for subsequent experiments.

In the procedure described in Sections 3.3 and 3.4, extract preparation was based on the methods proposed by Dong et al. and Zhang et al. [14,124]. Briefly, $1.5 \mathrm{~g}$ of rhodiola samples were mixed with $15 \mathrm{~mL}$ of $70 \%$ methanol. Then they are sonicated in a $300 \mathrm{~W}$ water bath (KQ-300DE ultrasonic cleaner, Kunshan Ultrasonic Instrument Co., LTD., Kunshan, China) for $30 \mathrm{~min}$ at room temperature with occasional stirring. The supernatant was collected after filtration by a $0.22 \mu \mathrm{m}$ filter. This extraction process was repeated twice more with the residue. Afterwards, these three supernatants were pooled together, and then stored at $-20^{\circ} \mathrm{C}$.

\subsection{Determination of Oxidative States and Ascorbic Acids}

On the basis of the protocols provided in corresponding Content Determination Kits (Solarbio Life Sciences, Beijing, China), the contents of $\mathrm{H}_{2} \mathrm{O}_{2}$, Protein carbonyl, $\mathrm{O}_{2}{ }^{--}, \mathrm{MDA}$, AsA (ascorbic acid), and DHA (dehydroascorbate) were determined using the colorimetric method. The contents of total ascorbic acids were calculated by the sum of AsA and DHA.

\subsection{Determination of Phenolic Components \\ 3.3.1. Total Phenols}

Following the Folin-Ciocalteau method proposed by Sun et al. [125], the total phenols were determined. In brief, $20 \mu \mathrm{L}$ extracts, standards (10-400 mg/L gallic acid) or blank (distilled water) were mixed with $40 \mu \mathrm{L}$ of $25 \%$ Folin-Ciocalteu, respectively, and then $140 \mu \mathrm{L}$ of $700 \mathrm{mM} \mathrm{Na}{ }_{2} \mathrm{CO}_{3}$ were added and shaken at $250 \mathrm{rpm}$ for $5 \mathrm{~min}$. After incubation for $2 \mathrm{~h}$ at room temperature in the dark, absorbances were measured at $765 \mathrm{~nm}$ using a microplate reader (Tecan Infinite 200 Pro Full Wavelength Microplate Reader, Tecan, Männedorf, Switzerland). The results were expressed as mg gallic acid/100 g d.w. of Rhodiola crenulata rhizomes.

\subsubsection{Total Tannins}

Total tannins were determined by casein-precipitation reaction based on the method proposed by Dong et al. [14]. In a nutshell, $8 \mathrm{~g}$ casein was added into $10 \mathrm{~mL}$ of the extract, and then the mixture was kept shaking for $3 \mathrm{~h}$ at $200 \mathrm{rpm}$. The mixture was subsequently placed in a $0.22-\mu \mathrm{m}$ filter to obtain filtrate. The phenolic acid in filtrate was determined by the method described in Section 3.3.1. A standard curve of gallic acid (10-400 mg/L) was established. Total tannins were calculated by the difference of phenols before and after casein-precipitation reaction.

\subsubsection{Total Flavonoids}

Total flavonoids were determined by the aluminum chloride colorimetric assay according to the approach developed by Dong et al. [14]. Briefly, $140 \mu \mathrm{L}$ extracts, standards (10-100 mg/L rutin) or blank (distilled water) were mixed with $8 \mu \mathrm{L}$ of $50 \mathrm{mg} / \mathrm{mL} \mathrm{NaNO}$ for $6 \mathrm{~min}$, respectively, followed by adding $8 \mu \mathrm{L}$ of $100 \mathrm{mg} / \mathrm{mL} \mathrm{AlCl} 3$. After a 5 -min standing, $100 \mu \mathrm{L}$ of $40 \mathrm{mg} / \mathrm{mL} \mathrm{NaOH}$ were added and incubated at room temperature for $30 \mathrm{~min}$. Absorbances were measured at $410 \mathrm{~nm}$ with the microplate reader. The results were expressed as mg rutin/100 g d.w. of Rhodiola crenulata rhizomes.

\subsubsection{Condensed Tannins}

Condensed tannins were detected using a vanillin assay as described by Fan et al. [126]. In short, $20 \mu \mathrm{L}$ extracts, standards (50-800 mg/L catechin) or blank (distilled water) were mixed with $120 \mu \mathrm{L}$ of $4 \%$ vanillin-methanol solution, respectively, and then $60 \mu \mathrm{L}$ of hydrochloric acid were added and incubated at room temperature for $15 \mathrm{~min}$. Subsequently, 
absorbances were measured at $500 \mathrm{~nm}$ with the microplate reader. The results were expressed as mg catechin/100 g d.w. of Rhodiola crenulata rhizomes.

\subsection{Determination of Antioxidant Capacity}

\subsubsection{DPPH-Scavenging Activity}

DPPH-scavenging activity was evaluated based on the method described in Dong et al. [14] In brief, $10 \mu \mathrm{L}$ extracts, standards (20-800 mg/L Trolox) or blank (distilled water) were mixed with $40 \mu \mathrm{L}$ of $1 \mathrm{mM} \mathrm{DPPH}$, respectively, and then $190 \mu \mathrm{L}$ methanol were added. After incubation for $30 \mathrm{~min}$ at room temperature in the dark, absorbances were measured at $517 \mathrm{~nm}$ using the microplate reader. Results were expressed as $\mathrm{mg}$ Trolox/100 g d.w. of Rhodiola crenulata rhizomes.

\subsection{2. $\mathrm{ABTS}^{+}$-Scavenging Activity}

$\mathrm{ABTS}^{+}$-scavenging activity was determined according to the method proposed in Dong et al. [14]. In short, ABTS solutions were prepared by mixing an equal volume of $7 \mathrm{mM}$ ABTS and $2.4 \mathrm{mM}$ potassium persulfate and incubated in dark at room temperature for 12-16 h. After incubation, the ABTS solution was diluted with methanol to an absorbance of $0.7 \pm 0.02$ at $734 \mathrm{~nm}$ to obtain ABTS working solution. Subsequently, $5 \mu \mathrm{L}$ of extracts, standards (20-800 mg/L Trolox) or blank (distilled water) were mixed with $200 \mu \mathrm{L}$ ABTS working solution, respectively. After incubation in dark at $30^{\circ} \mathrm{C}$ for $5 \mathrm{~min}$, absorbances were measured at $734 \mathrm{~nm}$ using the microplate reader. The results were expressed as mg Trolox/100 g d.w. of Rhodiola crenulata rhizomes.

\subsection{Mineral Content Analysis}

The contents of seven plant mineral elements in Rhodiola crenulata rhizomes were detected based on the method described in National Standard [127], including Ca, Sr, B, $\mathrm{Mn}, \mathrm{Ni}, \mathrm{Cu}$ and $\mathrm{Cd}(n=4)$. Briefly, $0.2 \mathrm{~g}$ of rhodiola sample was mixed with $3 \mathrm{~mL}$ nitric acid and $2 \mathrm{~mL}$ hydrochloric acid in a digestion tube, which was placed in a microwave digestion apparatus. The microwave digestion was performed according to the following gradient program: $0 \mathrm{~min}$ : room temperature; $0-5 \mathrm{~min}$ : linear increase to $130^{\circ} \mathrm{C} ; 5-13 \mathrm{~min}$ : $130{ }^{\circ} \mathrm{C}$ was maintained; $13-18 \mathrm{~min}$ : linear increase to $160^{\circ} \mathrm{C} ; 18-26 \mathrm{~min}: 160^{\circ} \mathrm{C}$ was kept; 26-31 min: linear increase to $195^{\circ} \mathrm{C}$; $31-61 \mathrm{~min}: 195^{\circ} \mathrm{C}$ was maintained; $61-62 \mathrm{~min}$ : linear decrease to $40{ }^{\circ} \mathrm{C}$. After digestion, the volume of the mixture was fixed to $50 \mathrm{~mL}$ with distilled water to obtain a test solution, and a blank solution was prepared in parallel.

$\mathrm{Ca}, \mathrm{Sr}, \mathrm{B}, \mathrm{Mn}, \mathrm{Ni}, \mathrm{Cu}$ and $\mathrm{Cd}$ were detected by inductively coupled plasma mass spectrometry (7850 ICP-MS, Agilent, Santa Clara, CA, USA). All elements were quantified by standard curves. The ICP-MS operation parameters were set as follows: RF power: $1600 \mathrm{~W}$; plasma flow: $14 \mathrm{~L} / \mathrm{min}$; auxiliary flow: $0.8 \mathrm{~L} / \mathrm{min}$; atomizing gas flow: $0.97 \mathrm{~L} / \mathrm{min}$; helium flow: $3 \mathrm{~mL} / \mathrm{min}$; sampling depth: $5 \mathrm{~mm}$; analog voltage: $-1820 \mathrm{~V}$; pulse voltage: $887 \mathrm{~V}$; acquisition time: $3 \mathrm{~s}$; spray chamber temperature: $2.7^{\circ} \mathrm{C}$.

\subsection{LC-ESI-MS/MS}

Rhodiola sample was prepared according to the method introduced in Section 3.1. To measure the assay's reproducibility, a QC (quality control) sample was obtained by mixing RC-L, RC-M, and RC-H.

Metabolite extraction and LC-ESI-MS/MS analysis were based on the method described in Fan et al. [128]. In brief, $50 \mathrm{mg}$ of rhodiola sample was mixd with $700 \mu \mathrm{L}$ of extract solution (methanol:water $=3: 1$, precooled at $-40^{\circ} \mathrm{C}$ ). Subsequently, the mixture was homogenized at $35 \mathrm{~Hz}$ for $4 \mathrm{~min}$ and sonicated in an ice-water bath for $5 \mathrm{~min}$. The homogenization and sonication cycle were repeated twice. The mixture was then further extracted overnight at $4{ }^{\circ} \mathrm{C}$ on a shaker. Following centrifugation at $13,800 \times g$ for $15 \mathrm{~min}$ at $4{ }^{\circ} \mathrm{C}$, the extract was filtrated through a $0.22 \mu \mathrm{m}$ microporous membrane to obtain a filtrate.

The filtrates were analyzed by a LC-ESI-MS/MS system (HPLC, EXION LC system, Sciex, Framingham, MA, USA; MS, SCIEX QTrap 6500+, Sciex, Framingham, MA, USA). Extracts were separated through a Waters ACQUITY UPLC HSS T3 C18 (1.8 $\mu \mathrm{m}$, 
$2.1 \times 100 \mathrm{~mm}$, Waters, Milford, MA, USA) as the column under following mobile phase conditions: mobile phase $\mathrm{A}$ was $0.1 \%$ formic acid in water, and mobile phase $\mathrm{B}$ was acetonitrile. The measurements were performed using the following gradient program: 0-0.5 min: $98 \%$ A, 2\% B; 0.5-11 min: a linear gradient to 5\% A, 95\% B; $11-13$ min: 5\% A plus $95 \%$ B was kept; a combination of $98 \%$ A with $2 \%$ B was set within 0.10 min and kept running for 13.1-15 $\mathrm{min}$. The column temperature was set at $40^{\circ} \mathrm{C}$. The auto-sampler temperature was set at $4{ }^{\circ} \mathrm{C}$ and the injection volume was $2 \mu \mathrm{L}$. The effluent was connected to an ESI-triple quadrupole-linear ion trap (QqQ-LIT)-MS.

The separated components were detected using a triple quadrupole (QqQ)-linear ion trap (LIT) mass spectrometer integrated with an IonDrive Turbo V ESI interface (SCIEX QTrap 6500+, Sciex, Framingham, MA, USA) and operated in positive and negative ion modes. The ESI source operation parameters were as follows: ion spray voltage: +5500 / $-4500 \mathrm{~V}$; curtain gas: $35 \mathrm{psi}$; temperature: $400{ }^{\circ} \mathrm{C}$; ion source gas 1:60 psi; ion source gas 2:60 psi; declustering potential (DP): $\pm 100 \mathrm{~V}$. Instrument tuning and mass calibration were performed in QqQ and LIT modes with polyethylene glycol solutions of $10 \mu \mathrm{mol} / \mathrm{L}$ and $100 \mu \mathrm{mol} / \mathrm{L}$, respectively. The QqQ scan was performed by multiple reaction monitoring (MRM) with the collision gas (nitrogen) set to 5 psi. The DP and CE of single MRM transfer were achieved by further optimization of declustering potential (DP) and collision energy (CE). Based on metabolites eluted during this period, a specific set of MRM transition was monitored in each cycle.

\subsection{Statistical Analysis}

Multivariate analysis was performed using the SIMCA16.0.2 software package (Sartorius Stedim Data Analytics AB, Umea, Sweden), including principal component analysis (PCA), orthogonal partial least squares discriminant analysis (OPLS-DA), hierarchical clustering analysis (HCA) and heat map construction. PCA is an unsupervised approach that reduces the dimensionality of the data and visualizes the sample distribution and grouping. 95\% confidence interval in the PCA score plot was used as a threshold to identify potential outliers in dataset. The data were processed by HCA to create a heatmap. Based on OPLS-DA, differential metabolites were evaluated by the values of variable importance in the projection (VIP), fold change (FC) and p-value, with the criteria of simultaneously meeting VIP $>1, \mid \log ^{2}$ (fold change) $\mid \geq 1$, and $p$-value $<0.05$. Biomarkers were identified by the high abundance of a metabolite in a Rhodiola sample versus zero abundance (set by $9.00 \mathrm{cps}$ ) of the corresponding metabolite in another Rhodiola sample.

\section{Conclusions}

In this paper, compared with ascorbic acid, the upregulation of phenolic components was more significant, along with the increased antioxidant capacities, in the response of Rhodiola crenulata to the altitude gradient, which may be the responses of Rhodiola crenulata to altitude-caused stresses.

It is also important to note regarding to the accumulation of six metabolites classes including phenolic components, and seven mineral elements related to stress resistance, this study proposes a novel approach to identify the elevation in the same mountain from 3933-4531 m leads to the following results: (1) overall upregulation of the top-down synthetic pathway of shikimic acid-phenylalanine-phenylpropanoids-flavonoids; (2) the number of upregulated biomarkers suggested that the upregulation of phenylpropanoids was more significant than that of flavonoids; (3) phenylpropanoids indirectly derived from Cinnamic acid-Coumaroyl via the phenylpropanoid pathway were more significantly affected, with phenylpropanes and phenylmethanes having a significant impact on the accumulation of phenylpropanoids; (4) the upregulation of quercetin and its derivatives was the most significant among the flavonoids; (5) the upregulation of condensed tannins and the downregulation of hydrolyzed tannins; (6) the significant upregulation of amino acids; (7) the overall increase in the accumulation of seven mineral elements related to resistance; 
(8) free fatty acids were significantly upregulated and glycerides were downregulated; and

(9) nucleotides were generally upregulated, with adenosine phosphates upregulated.

The above findings uncovered how Rhodiola crenulata upregulates phenolic components by enhancing the shikimic acid-phenylalanine-phenylpropanoids-flavonoids pathway; changes free fatty acids and glycerides, and nucleotides; and promotes the accumulation of seven mineral elements associated with resilience under extreme high altitude adversity. These findings provide new insights and new directions for the responses of Rhodiola crenulata to extreme high altitude adversities, and also offer an important basis for the in-depth development of the medicinal components of Rhodiola crenulata. More importantly, present study provided a rich and solid scientific support for further conducting research with different subjects.

Supplementary Materials: The following are available online: Table S1: List of identified metabolites between quality control, RC-L, RC-M, and RC-H; Table S2: List of differential metabolites between RC-H vs. RC-L; Table S3: List of differential metabolites between RC-M vs. RC-L; Table S4: List of differential metabolites between RC-H vs. RC-M; Figure S1: Heat map for a total of 1165 metabolites in RC-L, RC-M, and RC-H; Figure S2: OPLS-DA permutation plots; Figure S3: Heat map for recognized differential metabolites in RC-L, RC-M, and RC-H.

Author Contributions: Conceptualization, L.S.; methodology, L.S. and T.D.; software, T.D.; validation, L.S. and T.D.; formal analysis, L.S., T.D., Y.S. and H.L.; investigation, L.S., T.D., Y.S. and H.L.; resources, L.S.; data curation, L.S., T.D., Y.S. and H.L.; writing-original draft preparation, L.S. and T.D.; writing—-review and editing, L.S.; visualization, T.D.; supervision, L.S.; project administration, L.S.; funding acquisition, L.S. All authors have read and agreed to the published version of the manuscript.

Funding: This research was funded by the National Natural Science Foundation of China (grant No. 31800270), and the Fundamental Research Funds for the Central Universities (grant No. 2018ZY29). The APC was funded by the National Natural Science Foundation of China (grant No. 31800270).

Institutional Review Board Statement: Not applicable.

Informed Consent Statement: Not applicable.

Data Availability Statement: Data are available from the authors.

Acknowledgments: We sincerely thank Shubin Dong at Beijing Forestry University for the authentication of Rhodiola crenulata (Hook.f. \& Thomson) H.Ohba.

Conflicts of Interest: The authors declare no conflict of interest. The funders had no role in the design of the study; in the collection, analyses, or interpretation of data; in the writing of the manuscript, or in the decision to publish the results.

Sample Availability: Samples of the Rhodiola are available from the authors.

\begin{tabular}{ll}
\multicolumn{2}{l}{ Abbreviations } \\
1 (Figure 7) & Q3ORu \\
2 & 7OMQ \\
3 & Q7OR \\
4 & Q3ßDS \\
5 & Q3O(2OG)G \\
6 & Q3OGl \\
7 & Q3OS \\
8 & Q3OG $(1 \rightarrow 4)$ R7OR \\
9 & Q3O $(2 \mathrm{OR}) \mathrm{R}$ \\
10 & Q3O(6OA)G \\
11 & Q3O $(2 \mathrm{OG})$ glucuronide \\
12 & $5 \mathrm{OM}$ \\
13 & $\mathrm{I} 3 \mathrm{OR}$
\end{tabular}

\section{Abbreviations}

\author{
quercetin-3-O-rutinoside (Rutin) \\ 7-O-methxyl quercetin (Rhamnetin) \\ quercetin-7-O-rutinoside \\ quercetin 3- $\beta$-D-sophoroside \\ quercetin-3-O-(2"'-O-galactosyl)glucoside \\ quercetin-3-O-glucoside (Isoquercitrin) \\ quercetin-3-O-sophoroside (Baimaside) \\ quercetin-3-O-glucosyl $(1 \rightarrow 4)$ rhamnoside-7-O-rutinoside \\ quercetin-3-O-(2" $-\mathrm{O}-\mathrm{Rhamnosyl})$ rutinoside \\ quercetin-3-O-(6"-O-arabinosyl)glucoside \\ quercetin-3-O-(2"'-O-glucosyl)glucuronide \\ 5-O-methylquercetin (Azaleatin) \\ isorhamnetin-3-O-rutinoside
}




\begin{tabular}{|c|c|c|}
\hline 14 & R3OR & rhamnetin-3-O-rutinoside \\
\hline 15 & Q7OßDG & quercetin $7-\mathrm{O}-\beta$-D-glucoside \\
\hline 16 & Q3OGa & quercetin-3-O-galactoside (Hyperin) \\
\hline 17 & Q3OR7OG & quercetin-3-O-rutinoside-7-O-glucoside \\
\hline 18 & Q3OX & quercetin-3-O-xyloside (Reynoutrin) \\
\hline 19 & Q3ORo & quercetin-3-O-robinobioside \\
\hline 20 & $\mathrm{Q} 3 \mathrm{O}(6 \mathrm{G}) \mathrm{G}$ & quercetin-3-O-(6"'-galloyl)galactoside \\
\hline 21 (Figure 8) & D7OG & dihydrokaempferol-7-O-glucoside \\
\hline 22 & 6CM3G & 6-C-methylkaempferol-3-glucoside \\
\hline 23 & D3OG & dihydrokaempferol-3-O-glucoside \\
\hline 24 & $6 \mathrm{H} 36 \mathrm{OD}$ & 6-hydroxykaempferol-3,6-O-diglucoside \\
\hline 25 & K68DCG7OG & kaempferol-6,8-di-C-glucoside-7-O-glucoside \\
\hline 26 & K4OG & kaempferol-4'-O-glucoside \\
\hline 27 & K3OGa & kaempferol-3-O-galactoside (Trifolin) \\
\hline 28 & $\mathrm{~K} 3 \mathrm{ON}$ & kaempferol-3-O-neohesperidoside \\
\hline 29 (Figure 9) & $(-)-\mathrm{E} 3(3 \mathrm{OM}) \mathrm{G}$ & (-)-epicatechin-3-(3"I-O-methyl)gallate \\
\hline 30 & PC13OG & procyanidin $\mathrm{C} 13^{\prime}$-O-gallate \\
\hline 31 (Figure 10) & L3OG & luteolin-3'-O-glucoside \\
\hline 32 & L7O(2OR)R & luteolin-7-O-(2"'-O-rhamnosyl)rutinoside \\
\hline 33 (Figure 11) & 13TA & 1,3-trigallic acid \\
\hline 34 & $3 \mathrm{H} 5 \mathrm{M} 1 \mathrm{O}(6 \mathrm{D}) \mathrm{G}$ & 3-hydroxy-5-methylphenol-1-O-(6'-digalloyl) glucoside \\
\hline 35 & 3OMA & 3-O-methylgallic acid \\
\hline 36 & 17DOGDS & 1,7-di-O-galloyl-D-sedoheptulose \\
\hline 37 & M6OGG & methyl 6-O-galloyl-glucoside \\
\hline 38 & 2OS6OGDG & 2-O-salicyl-6-O-galloyl-D-glucose \\
\hline 39 & $\mathrm{MD}$ & monogalloyl-diglucose \\
\hline 40 & 14TA & 1,4-trigallic acid \\
\hline 41 & 23OD146TOGG & 2,3-O-digalloyl-1,4,6-tri-O-galloyl-glucose \\
\hline 42 & 1236TOGDG & 1,2,3,6-tetra-O-galloyl-D-glucose \\
\hline 43 & 2OGG & 2-O-galloyl-glucose \\
\hline 44 & 1OGDG & 1-O-galloyl-D-glucose \\
\hline 45 & 6OGG & 6-O-galloyl-glucose \\
\hline 46 & 16DOGDG & 1,6-di-O-galloyl-D-glucose \\
\hline 47 & 3OD1246OTDG & 3-O-digalloyl-1,2,4,6-O-tetragalloyl-D-glucose \\
\hline 48 (Figure 12) & CA & caffeoylbenzoyltartaric acid \\
\hline 49 & 12OD & 1,2-O-diferuloylglycerol \\
\hline 50 & CAME & chlorogenic acid methyl ester \\
\hline 51 & $\mathrm{SM}$ & sinapoyl malate \\
\hline 52 & $\mathrm{MC}$ & methyl caffeate \\
\hline 53 & 4HA4DG & 4-hydroxycinnamyl alcohol 4-D-glucoside \\
\hline 54 & 7M5P & 7-methoxy-5-prenyloxycoumarin \\
\hline 55 & SADG & syringoylcaffeoylquinic acid-D-glucose \\
\hline 56 & 3OPCAOG & 3-O-p-Coumaroylquinic acid O-glucoside \\
\hline 57 & SA & sinapic acid \\
\hline 58 & 1OGS & 1-O-glucosyl sinapate \\
\hline 59 & 1O[(E)PC]DG & 1-O-[(E)-p-coumaroyl]-D-glucose \\
\hline 60 & 3M48D34D & 3-methyl-4,8-dihydroxy-3,4-dihydroisocoumarin \\
\hline 61 & 6OFDG & 6-O-feruloyl-D-glucose \\
\hline 62 & PCA & p-coumaryl alcohol \\
\hline 63 & 7H5M & 7-hydroxy-5-methoxycoumarin \\
\hline 64 & $2 \mathrm{HA}$ & 2-hydroxycinnamic acid \\
\hline 65 & $3(4 \mathrm{H}) \mathrm{PA}$ & 3-(4-hydroxyphenyl)-propionic acid \\
\hline 66 & $\mathrm{C}(\mathrm{PH}) \mathrm{TA}$ & caffeoyl(p-hydroxybenzoyl)tartaric acid \\
\hline 67 & $3(3 \mathrm{H}) \mathrm{PA}$ & 3-(3-hydroxyphenyl)-propionate acid \\
\hline
\end{tabular}




\begin{tabular}{|c|c|c|}
\hline 68 & $(\mathrm{~S}) 2 \mathrm{H} 3(4 \mathrm{H}) \mathrm{PA}$ & (S)-2-hydroxy-3-(4-hydroxyphenyl)propanoic acid \\
\hline 69 & PA & phaseolic acid \\
\hline 70 & FA & ferulic acid \\
\hline 71 & $\mathrm{BCA}$ & brevifolin carboxylic acid \\
\hline 72 & MCA & maleoyl-caffeoylquinic acid \\
\hline 73 & 57D & 5,7-dimethoxycoumarin \\
\hline 74 & 3ОРСA & 3-O-p-coumaroylquinic acid \\
\hline 75 & NA & neochlorogenic acid(5-O-caffeoylquinic acid) \\
\hline 76 & $1 \mathrm{O}(34 \mathrm{D}) \mathrm{OCG}$ & 1'-O-(3,4-dihydroxyphenethyl)-O-caffeoyl-glucoside \\
\hline 77 & $\mathrm{M} 4 \mathrm{H}$ & methyl 4-hydroxycinnamate \\
\hline 78 (Figure 13) & $23456 \mathrm{P} 2 \mathrm{H}$ & 2,3,4,5,6-pentahydroxyhexyl 2-hydroxybenzoate \\
\hline 79 & $1 \mathrm{O}(34 \mathrm{D} 5 \mathrm{MB}) \mathrm{G}$ & 1-O-(3,4-dihydroxy-5-methoxy-benzoyl)-glucoside \\
\hline 80 & VA4OG & vanillic acid-4-O-glucoside \\
\hline 81 & PA4OG & protocatechuic acid-4-O-glucoside \\
\hline 82 & 345T1OG & 3,4,5-trimethoxyphenyl-1-O-glucoside \\
\hline 83 & 35D4MA & 3,5-dihydroxy-4-methoxybenzoic acid \\
\hline 84 & 25DAOG & 2,5-dihydroxybenzoic acid O-glucoside \\
\hline 85 & 1OVDG & 1-O-vanilloyl-D-glucose \\
\hline 86 & $3 \mathrm{H}$ & 3-hydroxybenzaldehyde \\
\hline 87 & $4 \mathrm{HA}$ & 4-hydroxybenzoic acid \\
\hline 88 & SA2OG & salicylic acid-2-O-glucoside \\
\hline 89 & SA & syringic acid \\
\hline 90 & $4 \mathrm{H}$ & 4-hydroxybenzaldehyde \\
\hline 91 & 4OG4HA & 4-O-glucosyl-4-hydroxybenzoic acid \\
\hline 92 & $\mathrm{DP}$ & dimethyl phthalate \\
\hline 93 & PA & protocatechuic aldehyde \\
\hline 94 & 3ODQA & 3-O-digalloyl quinic acid \\
\hline 95 & 246TA & 2,4,6-trihydroxybenzoic acid \\
\hline 96 (Figure 14) & $4 \mathrm{H}$ & 4-hydroxyacetophenone \\
\hline 97 & PHAA & p-hydroxyphenyl acetic acid \\
\hline 98 & 3H4I3OG & 3-hydroxy-4-isopropylbenzylalcohol-3-O-glucoside \\
\hline 99 & M24D & methyl 2,4-dihydroxyphenylacetate \\
\hline 100 & $2 \mathrm{PD} \beta \mathrm{G}$ & 2-phenylethyl-D- $\beta$-glucopyranoside \\
\hline 101 & 24D6M & $2^{\prime}, 4^{\prime}$-dihydroxy-6'-methoxyacetophenone \\
\hline 102 & 34DA & 3,4-dihydroxybenzeneacetic acid \\
\hline 103 (Figure 15) & 35DOGA & 3,5-di-O-galloylshikimic acid \\
\hline 104 & 3GA & 3-galloylshikimic acid \\
\hline 105 & $\mathrm{~T} 5 \mathrm{O}(\mathrm{PC}) \mathrm{S}$ & trans-5-O-(p-coumaroyl) shikimate \\
\hline 106 & $50 C A$ & 5-O-caffeoylshikimic acid \\
\hline 107 (Figure 16) & (5LG)LAA & (5-L-glutamyl)-L-amino acid \\
\hline 108 & $\mathrm{~N} \alpha \mathrm{ALO}$ & $\mathrm{N}$ - $\alpha$-acetyl-L-ornithine \\
\hline 109 & 5OLP & 5-oxo-L-proline \\
\hline 110 & T4HLP & trans-4-hydroxy-L-proline \\
\hline 111 & NALM & N-acetyl-L-methionine \\
\hline 112 & LILA & L-isoleucyl-L-aspartate \\
\hline 113 & LGLI & L-glycyl-L-isoleucine \\
\hline 114 & LAAOD & L-aspartic acid-O-diglucoside \\
\hline 115 & NALAA & N-acetyl-L-aspartic acid \\
\hline 116 & LAsLP & L-aspartyl-L-phenylalanine \\
\hline 117 & LGLP & L-glycyl-L-phenylalanine \\
\hline 118 & 5HLT & 5-hydroxy-L-tryptophan \\
\hline 119 & NALT & $\mathrm{N}$-acetyl-L-tryptophan \\
\hline 120 & LPLP & L-prolyl-L-phenylalanine \\
\hline 121 & $\mathrm{~S}(5 \mathrm{~A}) \mathrm{LH}$ & S-(5'-adenosy)-L-homocysteine \\
\hline
\end{tabular}




\begin{tabular}{|c|c|c|}
\hline 122 & $\mathrm{~S}(\mathrm{M}) \mathrm{G}$ & S-(methyl)glutathione \\
\hline 123 & NND & N,N-dimethylglycine \\
\hline 124 & $\mathrm{~N}(3 \mathrm{I}) \mathrm{LA}$ & N-(3-indolylacetyl)-L-alanine \\
\hline 125 & LPLL & L-prolyl-L-leucine \\
\hline 126 & NGLL & N-glycyl-L-leucine \\
\hline 127 & NALGA & $\mathrm{N}$-acetyl-L-glutamic acid \\
\hline 128 & NALG & N-acetyl-L-glutamine \\
\hline 129 & 3H3M15DA & 3-hydroxy-3-methylpentane-1,5-dioic acid \\
\hline 130 & LGOG & L-glutamine-O-glycoside \\
\hline 131 & L $\alpha$ GLGA & L- $\alpha$-glutamyl-L-glutamic acid \\
\hline 132 & LGAOG & L-glutamic acid-O-glycoside \\
\hline 133 & $\mathrm{AM}$ & acetylleucine monoethanolamine \\
\hline 134 & 34DLP & 3,4-dihydroxy-L-phenylalanine \\
\hline 135 & NALL & N-acetyl-L-leucine \\
\hline 136 & NALA & N-acetyl-L-arginine \\
\hline 137 & LAILP & L-alanyl-L-phenylalanine \\
\hline 138 & 3MLH & 3-methyl-L-histidine \\
\hline 139 (Figure 17) & 910EA & 9,10-epoxyoctadecanoic acid \\
\hline 140 & 91013T11OA & 9,10,13-trihydroxy-11-octadecenoic acid \\
\hline 141 & 9H12O15(Z)OA & 9-hydroxy-12-oxo-15(Z)-octadecenoic acid \\
\hline 142 & 91213T1015OA & 9,12,13-trihydroxy-10,15-octadecadienoic acid \\
\hline 143 & 9H13O10OA & 9-hydroxy-13-oxo-10-octadecenoic acid \\
\hline 144 & 1213E9OA & 12,13-epoxy-9-octadecenoic acid \\
\hline 145 & 13-KODE & (9Z,11E)-13-oxooctadeca-9,11-dienoic acid \\
\hline 146 & 12,13-DHOME & (9Z)-12,13-dihydroxyoctadec-9-enoic acid \\
\hline 147 & 9H101215OA & 9-hydroxy-10,12,15-octadecatrienoic acid \\
\hline 148 & 13SH9Z11EOA & 13S-hydroperoxy-9Z,11E-octadecadienoic acid \\
\hline 149 & PA & punicic acid \\
\hline 150 & 9O10E12ZOA & 9-oxo-10E,12Z-octadecadienoic acid \\
\hline 151 & 2L1OG & 2-linoleoylglycerol-1-O-glucoside \\
\hline 152 & $2 \alpha$ LG13DOG & 2- $\alpha$-linolenoyl-glycerol-1,3-di-O-glucoside \\
\hline 153 & $1 \alpha \mathrm{LG} 3 \mathrm{OG}$ & 1- $\alpha$-linolenoyl-glycerol-3-O-glucoside \\
\hline 154 & 1LRGD & 1-linolenoyl-rac-glycerol-diglucoside \\
\hline 155 & 1SG & 1-stearidonoyl-glycerol \\
\hline 156 & $2 \alpha \mathrm{LG} 1 \mathrm{OG}$ & 2- $\alpha$-linolenoyl-glycerol-1-O-glucoside \\
\hline 157 & 815D591113EA & 8,15-dihydroxy-5,9,11,13-eicosatetraenoic acid \\
\hline 158 & C4710131619DA & cis- $4,7,10,13,16,19$-docosahexaenoic acid \\
\hline 159 & $\mathrm{C} 10 \mathrm{HA}$ & cis-10-heptadecenoic acid \\
\hline 160 & 9(10)-EpOME & 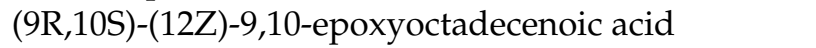 \\
\hline 161 & HA & heptadecanoic acid \\
\hline 162 & 13(S)-HODE & 13(S)-hydroxyoctadeca-9Z,11E-dienoic acid \\
\hline 163 & 9SH10E12ZOA & 9S-hydroxy-10E,12Z-octadecadienoic acid \\
\hline 164 & 912O6YA & 9,12-octadecadien-6-ynoic acid \\
\hline 165 & EA & eicosadienoic acid \\
\hline 166 & 9H10E1215ZOA & 9-hydroperoxy-10E,12,15Z-octadecatrienoic acid \\
\hline 167 & VA & vaccenic acid \\
\hline 168 & AA & arachidonic acid \\
\hline 169 & $\gamma \mathrm{LA}$ & $\gamma$-linolenic acid \\
\hline 170 & $\alpha \mathrm{LA}$ & $\alpha$-linolenic acid \\
\hline 171 & $1 \alpha \mathrm{LG} 23 \mathrm{DOG}$ & 1- $\alpha$-linolenoyl-glycerol-2,3-di-O-glucoside \\
\hline 172 & 1L23DOG & 1-linoleoylglycerol-2,3-di-O-glucoside \\
\hline 173 & 7S,8S-DiHODE & (9Z,12Z)-(7S,8S)-dihydroxyoctadeca-9,12-dienoic acid \\
\hline 174 & 2L13DOG & 2-linoleoylglycerol-1,3-di-O-glucoside \\
\hline 175 & 1OSG & 1-oleoyl-sn-glycerol \\
\hline
\end{tabular}




$\begin{array}{lll}176 \text { (Figure 18) } & \text { A5M } & \text { adenosine } 5^{\prime} \text {-monophosphate } \\ 177 & \text { 2D1P } & \text { 2-deoxyribose-1-phosphate } \\ 178 & \text { 6M } & \text { 6-methylmercaptopurine } \\ 179 & \text { I7NG } & \text { isopentenyladenine-7-N-glucoside } \\ 180 & \text { A5D } & \text { adenosine 5'-diphosphate } \\ 181 & \text { 5AR } & \text { 5-aminoimidazole ribonucleotide } \\ 182 & \text { U5M } & \text { uridine 5'-monophosphate } \\ 183 & \text { N6I } & \text { N6-isopentenyladenine } \\ 184 & \text { NDR } & \text { nicotinate D-ribonucleoside } \\ 185 & \text { NADP } & \text { nicotinamide adenine dinucleotide phosphate } \\ 186 & \text { 2D5M } & \text { 2'-deoxyadenosine-5'-monophosphate } \\ 187 & \text { 9(A)H } & \text { 9-(arabinosyl)hypoxanthine } \\ 188 & \text { G5M } & \text { guanosine 5'-monophosphate }\end{array}$

\section{References}

1. Panossian, A.; Wikman, G.; Sarris, J. Rosenroot (Rhodiola rosea): Traditional use, chemical composition, pharmacology and clinical efficacy. Phytomedicine 2010, 17, 481-493. [CrossRef]

2. Panossian, A.G.; Efferth, T.; Shikov, A.N.; Pozharitskaya, O.N.; Kuchta, K.; Mukherjee, P.K.; Banerjee, S.; Heinrich, M.; Wu, W.; Guo, D.; et al. Evolution of the adaptogenic concept from traditional use to medical systems: Pharmacology of stress- and aging-related diseases. Med. Res. Rev. 2021, 41, 630-703. [CrossRef]

3. Shikov, A.N.; Narkevich, I.A.; Flisyuk, E.V.; Luzhanin, V.G.; Pozharitskaya, O.N. Medicinal plants from the 14th edition of the Russian Pharmacopoeia, recent updates. J. Ethnopharmacol. 2021, 268, 113685. [CrossRef]

4. Tao, H.; Wu, X.; Cao, J.; Peng, Y.; Wang, A.; Pei, J.; Xiao, J.; Wang, S.; Wang, Y. Rhodiola species: A comprehensive review of traditional use, phytochemistry, pharmacology, toxicity, and clinical study. Med. Res. Rev. 2019, 39, 1779-1850. [CrossRef]

5. Chinese Pharmacopoeia Commission. Pharmacopoeia of People's Republic of China; People's Medical Publishing House: Beijing, China, 2005; pp. 211-212. ISBN 7-117-06982-1/R 6983.

6. Booker, A.; Zhai, L.; Gkouva, C.; Li, S.; Heinrich, M. From traditional resource to global commodities-A comparison of Rhodiola species using NMR spectroscopy-Metabolomics and HPTLC. Front. Pharmacol. 2016, 7, 254. [CrossRef]

7. Recio, M.-C.; Giner, R.; Máñez, S. Immunmodulatory and antiproliferative properties of Rhodiola species. Planta Med. 2016, 82, 952-960. [CrossRef] [PubMed]

8. Cui, J.-L.; Guo, T.-T.; Ren, Z.-X.; Zhang, N.-S.; Wang, M.-L. Diversity and antioxidant activity of culturable Endophytic fungi from Alpine plants of Rhodiola crenulata, R. angusta, and R. sachalinensis. PLoS ONE 2015, 10, e0118204. [CrossRef]

9. Bassa, L.M.; Jacobs, C.; Gregory, K.; Henchey, E.; Ser-Dolansky, J.; Schneider, S.S. Rhodiola crenulata induces an early estrogenic response and reduces proliferation and tumorsphere formation over time in MCF7 breast cancer cells. Phytomedicine 2016, 23, 87-94. [CrossRef]

10. Hsiao, Y.-W.; Tsai, Y.-N.; Huang, Y.-T.; Liu, S.-H.; Lin, Y.-J.; Lo, L.-W.; Hu, Y.-F.; Chung, F.-P.; Lin, S.-F.; Chang, S.-L.; et al. Rhodiola crenulata reduces ventricular arrhythmia through mitigating the activation of IL-17 and inhibiting the MAPK signaling pathway. Cardiovasc. Drugs Ther. 2020, 35, 1-12. [CrossRef]

11. Chiu, T.-F.; Chen, L.L.-C.; Su, D.-H.; Lo, H.-Y.; Chen, C.-H.; Wang, S.-H.; Chen, W.-L. Rhodiola crenulata extract for prevention of acute mountain sickness: A randomized, double-blind, placebo-controlled, crossover trial. BMC Complement. Altern. Med. 2013, 13, 298. [CrossRef] [PubMed]

12. Wang, L.; Wang, Y.; Yang, W.; He, X.; Xu, S.; Liu, X.; He, Y.; Hu, Q.; Yuan, D.; Jin, T. Network pharmacology and molecular docking analysis on mechanisms of Tibetan Hongjingtian (Rhodiola crenulata) in the treatment of COVID-19. J. Med. Microbiol. 2021, 70, 001374. [CrossRef] [PubMed]

13. Chuang, M.-L.; Wu, T.-C.; Wang, Y.-T.; Wang, Y.-C.; Tsao, T.C.-Y.; Wei, J.C.-C.; Chen, C.-Y.; Lin, I.-F. Adjunctive treatment with Rhodiola Crenulata in patients with chronic obstructive pulmonary disease-A randomized placebo controlled double blind clinical trial. PLoS ONE 2015, 10, e0128142. [CrossRef] [PubMed]

14. Dong, X.; Guo, Y.; Xiong, C.; Sun, L. Evaluation of two major Rhodiola species and the systemic changing characteristics of metabolites of Rhodiola crenulata in different altitudes by chemical methods combined with UPLC-QqQ-MS-based metabolomics. Molecules 2020, 25, 4062. [CrossRef] [PubMed]

15. Rhodiola Crenulata. Available online: http://www.iplant.cn/info/Rhodiola\%20crenulata?t=foc (accessed on 28 October 2021).

16. Oscar, V.; Monica, B. Flavonoids: Antioxidant compounds for plant defence. and for a healthy human diet. Not. Bot. Horti Agrobot. Cluj Napoca 2017, 46, 14-21.

17. Tattini, M.; Loreto, F.; Fini, A.; Guidi, L.; Brunetti, C.; Velikova, V.; Gori, A.; Ferrini, F. Isoprenoids and phenylpropanoids are part of the antioxidant defense orchestrated daily by drought-stressed Platanus $\times$ acerifolia plants during Mediterranean summers. New Phytol. 2015, 207, 613-626. [CrossRef]

18. Hagerman, A.E.; Riedl, K.M.; Jones, G.A.; Sovik, K.N.; Ritchard, N.T.; Hartzfeld, A.P.W.; Riechel, T.L. High molecular weight plant Polyphenolics (Tannins) as biological antioxidants. J. Agric. Food Chem. 1998, 46, 1887-1892. [CrossRef] 
19. Wang, Y.; Zhao, H.; Qin, H.; Li, Z.; Liu, H.; Wang, J.; Zhang, H.; Quan, R.; Huang, R.; Zhang, Z. The synthesis of ascorbic acid in rice roots plays an important role in the salt tolerance of rice by scavenging ROS. Int. J. Mol. Sci. 2018, 19, 3347. [CrossRef]

20. Hancock, R.; Viola, R. Biosynthesis and catabolism of L-Ascorbic acid in plants. Crit. Rev. Plant Sci. 2005, 24, 167-188. [CrossRef]

21. Xu, C.; Wei, L.; Huang, S.; Yang, C.; Wang, Y.; Yuan, H.; Xu, Q.; Zhang, W.; Wang, M.; Zeng, X.; et al. Drought resistance in Qingke involves a reprogramming of the Phenylpropanoid Pathway and UDP-Glucosyltransferase regulation of abiotic stress tolerance targeting Flavonoid Biosynthesis. J. Agric. Food Chem. 2021, 69, 3992-4005. [CrossRef] [PubMed]

22. Geng, D.; Shen, X.; Xie, Y.; Yang, Y.; Bian, R.; Gao, Y.; Li, P.; Sun, L.; Feng, H.; Ma, F.; et al. Regulation of phenylpropanoid biosynthesis by MdMYB88 and MdMYB124 contributes to pathogen and drought resistance in apple. Hortic. Res. 2020, 7, 1-11. [CrossRef] [PubMed]

23. Clemente-Moreno, M.J.; Omranian, N.; Sáez, P.L.; Figueroa, C.M.; Del-Saz, N.F.; Elso, M.; Poblete, L.; Orf, I.; Cuadros-Inostroza, A.; Cavieres, L.A.; et al. Low-temperature tolerance of the Antarctic species Deschampsia antarctica: A complex metabolic response associated with nutrient remobilization. Plant Cell Environ. 2020, 43, 1376-1393. [CrossRef]

24. Zhong, Z.; Liu, S.; Zhu, W.; Ou, Y.; Yamaguchi, H.; Hitachi, K.; Tsuchida, K.; Tian, J.; Komatsu, S. Phosphoproteomics reveals the biosynthesis of secondary metabolites in Catharanthus roseus under Ultraviolet-B radiation. J. Proteome Res. 2019, 18, 3328-3341. [CrossRef]

25. Zhu, L.-J.; Deng, X.-G.; Zhang, D.-W.; Lin, H.-H. Enhancement of stress tolerance in cucumber seedlings by proanthocyanidins. Biol. Plant 2017, 61, 323-332. [CrossRef]

26. Wilson, K.E.; Wilson, M.I.; Greenberg, B.M. Identification of the Flavonoid Glycosides that accumulate in Brassica napus L. cv. Topas specifically in response to ultraviolet B radiation. Photochem. Photobiol. 1998, 67, 547-553. [CrossRef]

27. Ortega, E.; Nair, V.; Welti-Chanes, J.; Cisneros-Zevallos, L.; Jacobo-Velázquez, D.A. Wounding and UVB light Synergistically induce the Biosynthesis of Phenolic compounds and Ascorbic Acid in Red Prickly Pears (Opuntia ficus-indica cv. Rojo Vigor). Int. J. Mol. Sci. 2019, 20, 5327. [CrossRef]

28. Xiang, N.; Hu, J.; Wen, T.; Brennan, M.A.; Brennan, C.S.; Guo, X. Effects of temperature stress on the accumulation of ascorbic acid and folates in sweet corn (Zea mays L.) seedlings. J. Sci. Food Agric. 2019, 100, 1694-1701. [CrossRef]

29. Varela, M.C.; Arslan, I.; Reginato, M.A.; Cenzano, A.M.; Luna, M.V. Phenolic compounds as indicators of drought resistance in shrubs from Patagonian shrublands (Argentina). Plant Physiol. Biochem. 2016, 104, 81-91. [CrossRef]

30. Amir, R.; Galili, G.; Cohen, H. The metabolic roles of free amino acids during seed development. Plant Sci. 2018, 275, 11-18. [CrossRef]

31. Moe, L.A. Amino acids in the rhizosphere: From plants to microbes. Am. J. Bot. 2013, 100, 1692-1705. [CrossRef]

32. Harding, H.; Zhang, Y.; Zeng, H.; Novoa, I.; Lu, P.D.; Calfon, M.; Sadri, N.; Yun, C.; Popko, B.; Paules, R.S.; et al. An integrated stress response regulates Amino Acid metabolism and resistance to oxidative stress. Mol. Cell 2003, 11, 619-633. [CrossRef]

33. Nehela, Y.; Killiny, N. 'Candidatus Liberibacter asiaticus' and its vector, Diaphorina citri, Augment the Tricarboxylic acid cycle of their host via the $\gamma$-Aminobutyric acid shunt and polyamines pathway. Mol. Plant Microbe Interact. 2019, 32, 413-427. [CrossRef]

34. Cheng, B.; Li, Z.; Liang, L.; Cao, Y.; Zeng, W.; Zhang, X.; Ma, X.; Huang, L.; Nie, G.; Liu, W.; et al. The $\gamma$-Aminobutyric acid (GABA) Alleviates salt stress damage during seeds germination of white clover associated with $\mathrm{Na}+/ \mathrm{K}+$ transportation, Dehydrins accumulation, and stress-related genes expression in white clover. Int. J. Mol. Sci. 2018, 19, 2520. [CrossRef]

35. Adams, Z.; Ehlting, J.; Edwards, R. The regulatory role of shikimate in plant phenylalanine metabolism. J. Theor. Biol. 2019, 462, 158-170. [CrossRef]

36. Manela, N.; Oliva, M.; Ovadia, R.; Sikron-Persi, N.; Ayenew, B.; Fait, A.; Galili, G.; Perl, A.; Weiss, D.; Oren-Shamir, M. Phenylalanine and tyrosine levels are rate-limiting factors in production of health promoting metabolites in Vitis vinifera cv. Gamay Red cell suspension. Front. Plant Sci. 2015, 6, 538. [CrossRef]

37. Taiz, L.; Zeiger, E. Chapter 13: Secondary metabolites and plant defense. In Plant Physiology, 4th ed.; Sinauer Associates, Inc.: Sunderland, MA, USA, 2006; pp. 290-292. ISBN 9780878938568.

38. Acuña-Avila, P.E.; Vásquez-Murrieta, M.S.; Hernández, M.O.F.; López-Cortéz, M.D.S. Relationship between the elemental composition of grapeyards and bioactive compounds in the Cabernet Sauvignon grapes Vitis vinifera harvested in Mexico. Food Chem. 2016, 203, 79-85. [CrossRef]

39. Martins, V.; Garcia, A.; Costa, C.; Sottomayor, M.; Gerós, H. Calcium- and hormone-driven regulation of secondary metabolism and cell wall enzymes in grape berry cells. J. Plant Physiol. 2018, 231, 57-67. [CrossRef] [PubMed]

40. Langer, S.E.; Marina, M.; Burgos, J.L.; Martínez, G.A.; Civello, P.M.; Villarreal, N.M. Calcium chloride treatment modifies cell wall metabolism and activates defense responses in strawberry fruit (Fragaria $\times$ ananassa, Duch). J. Sci. Food Agric. 2019, 99, 4003-4010. [CrossRef]

41. Kováčik, J.; Klejdus, B.; Bačkor, M. Phenolic metabolism of Matricaria chamomilla plants exposed to nickel. J. Plant Physiol. 2009, 166, 1460-1464. [CrossRef] [PubMed]

42. Wu, X.; Song, H.; Guan, C.; Zhang, Z. Boron mitigates cadmium toxicity to rapeseed (Brassica napus) shoots by relieving oxidative stress and enhancing cadmium chelation onto cell walls. Environ. Pollut. 2020, 263, 114546. [CrossRef]

43. Hanaka, A.; Dresler, S.; Wójciak-Kosior, M.; Strzemski, M.; Kováčik, J.; Latalski, M.; Zawiślak, G.; Sowa, I. the impact of long-and short-term Strontium treatment on metabolites and minerals in Glycine max. Molecules 2019, 24, 3825. [CrossRef]

44. Han, T.; Sun, M.; Zhao, J.; Dai, C.; Li, Y.; Zhang, P.; Lang, D.; Zhou, J.; Li, X.; Ge, S. The roles of cadmium on growth of seedlings by analysing the composition of metabolites in pumpkin tissues. Ecotoxicol. Environ. Saf. 2021, 226, 112817. [CrossRef] [PubMed] 
45. Li, R.; Zhou, X.; Liu, D.; Feng, W. Enhancing the activity and stability of Mn-superoxide dismutase by one-by-one ligation to catalase. Free. Radic. Biol. Med. 2018, 129, 138-145. [CrossRef]

46. Robinett, N.G.; Peterson, R.L.; Culotta, V.C. Eukaryotic copper-only superoxide dismutases (SODs): A new class of SOD enzymes and SOD-like protein domains. J. Biol. Chem. 2018, 293, 4636-4643. [CrossRef]

47. Kumar, R.R.; Dubey, K.; Goswami, S.; Hasija, S.; Pandey, R.; Singh, P.K.; Singh, B.; Sareen, S.; Rai, G.K.; Singh, G.P.; et al. Heterologous expression and characterization of novel manganese superoxide dismutase (Mn-SOD)—A potential biochemical marker for heat stress-tolerance in wheat (Triticum aestivum). Int. J. Biol. Macromol. 2020, 161, 1029-1039. [CrossRef] [PubMed]

48. Carvalho, M.E.; Castro, P.R.; Azevedo, R.A. Hormesis in plants under Cd exposure: From toxic to beneficial element? J. Hazard. Mater. 2020, 384, 121434. [CrossRef]

49. Singh, D.P.; Liu, L.H.; Øiseth, S.K.; Beloy, J.; Lundin, L.; Gidley, M.J.; Day, L. Influence of boron on carrot cell wall structure and its resistance to fracture. J. Agric. Food Chem. 2010, 58, 9181-9189. [CrossRef] [PubMed]

50. Song, W.-Y.; Zhang, Z.-B.; Shao, H.-B.; Guo, X.-L.; Cao, H.-X.; Zhao, H.-B.; Fu, Z.-Y.; Hu, X.-J. Relationship between calcium decoding elements and plant abiotic-stress resistance. Int. J. Biol. Sci. 2008, 4, 116-125. [CrossRef] [PubMed]

51. Martens, S.N.; Boyd, R.S. The ecological significance of nickel hyperaccumulation: A plant chemical defense. Oecologia 1994, 98, 379-384. [CrossRef]

52. Peterson, R.L.; Galaleldeen, A.; Villarreal, J.; Taylor, A.B.; Cabelli, D.E.; Hart, P.J.; Culotta, V.C. The phylogeny and active site design of Eukaryotic Copper-only superoxide dismutases. J. Biol. Chem. 2016, 291, 20911-20923. [CrossRef]

53. He, M.; Ding, N.-Z. Plant unsaturated fatty acids: Multiple roles in stress response. Front. Plant Sci. 2020, 11, 562785. [CrossRef]

54. Cao, Y.; Tanaka, K.; Nguyen, C.; Stacey, G. Extracellular ATP is a central signaling molecule in plant stress responses. Curr. Opin. Plant Biol. 2014, 20, 82-87. [CrossRef]

55. Gao, L.; Chen, W.; Xu, X.; Zhang, J.; Singh, T.; Liu, S.; Zhang, D.; Tian, L.; White, A.; Shrestha, P.; et al. Engineering Trienoic fatty acids into Cottonseed oil improves low-temperature seed germination, plant photosynthesis and cotton fiber quality. Plant Cell Physiol. 2020, 61, 1335-1347. [CrossRef]

56. Kalinger, R.S.; Pulsifer, I.P.; Hepworth, S.R.; Rowland, O. Fatty acyl synthetases and Thioesterases in plant lipid metabolism: Diverse functions and biotechnological applications. Lipids 2020, 55, 435-455. [CrossRef]

57. De Col, V.; Fuchs, P.; Nietzel, T.; Elsässer, M.; Voon, C.P.; Candeo, A.; Seeliger, I.; Fricker, M.D.; Grefen, C.; Møller, I.M.; et al. ATP sensing in living plant cells reveals tissue gradients and stress dynamics of energy physiology. eLife 2017, 6. [CrossRef]

58. Chen, Z.; He, C.; Hu, H. Temperature responses of growth, photosynthesis, fatty acid and nitrate reductase in Antarctic and temperate Stichococcus. Extremophiles 2011, 16, 127-133. [CrossRef]

59. Baâtour, O.; Kaddour, R.; Mahmoudi, H.; Tarchoun, I.; Bettaieb, I.; Nasri, N.; Mrah, S.; Hamdaoui, G.; Lachaâl, M.; Marzouk, B. Salt effects on Origanum majorana fatty acid and essential oil composition. J. Sci. Food Agric. 2011, 91, 2613-2620. [CrossRef]

60. Yu, P.; Jiang, N.; Fu, W.; Zheng, G.; Li, G.; Feng, B.; Chen, T.; Ma, J.; Li, H.; Tao, L.; et al. ATP Hydrolysis determines cold tolerance by regulating available energy for Glutathione synthesis in rice seedling plants. Rice 2020, 13, 1-16. [CrossRef]

61. Bajerski, F.; Stock, J.; Hanf, B.; Darienko, T.; Heine-Dobbernack, E.; Lorenz, M.; Naujox, L.; Keller, E.R.J.; Schumacher, H.M.; Friedl, T.; et al. ATP content and cell viability as indicators for Cryostress across the diversity of life. Front. Physiol. 2018, 9, 921. [CrossRef]

62. Zhu, W.; Miao, Q.; Sun, D.; Yang, G.; Wu, C.; Huang, J.; Zheng, C. The mitochondrial phosphate transporters modulate plant responses to salt stress via affecting ATP and Gibberellin metabolism in Arabidopsis thaliana. PLoS ONE 2012, 7, e43530. [CrossRef]

63. Reyes, T.H.; Esparza, E.; Crestani, G.; Limonchi, F.; Cruz, R.; Salinas, N.; Scartazza, A.; Guglielminetti, L.; Cosio, E. Physiological responses of maca (Lepidium meyenii Walp.) plants to UV radiation in its high-altitude mountain ecosystem. Sci. Rep. 2020, 10, 1-13. [CrossRef]

64. Cui, G.; Wei, X.; Degen, A.A.; Wei, X.; Zhou, J.; Ding, L.; Shang, Z.; Liu, S.; Long, R. Trolox-equivalent antioxidant capacity and composition of five alpine plant species growing at different elevations on the Qinghai-Tibetan Plateau. Plant Ecol. Divers. 2016, 9, 387-396. [CrossRef]

65. Neuner, G. Frost resistance in alpine woody plants. Front. Plant Sci. 2014, 5, 654. [CrossRef]

66. Mehla, N.; Sindhi, V.; Josula, D.; Bisht, P.; Wani, S.H. Reactive Oxygen Species and Antioxidant Systems in Plants: Role and Regulation under Abiotic Stress; Khan, M.I.R., Khan, N.A., Eds.; Springer: Singapore, 2017; pp. 1-23. ISBN 978-981-10-5253-8.

67. Alché, J.D.D. A concise appraisal of lipid oxidation and lipoxidation in higher plants. Redox Biol. 2019, 23, 101136. [CrossRef]

68. Sytykiewicz, H.; Łukasik, I.; Golawska, S.; Chrzanowski, G. Aphid-triggered changes in oxidative damage markers of nucleic acids, proteins, and lipids in Maize (Zea mays L.) seedlings. Int. J. Mol. Sci. 2019, 20, 3742. [CrossRef]

69. Ciacka, K.; Tymiński, M.; Gniazdowska, A.; Krasuska, U. Carbonylation of proteins-An element of plant ageing. Planta 2020, 252, 1-13. [CrossRef]

70. Singh, P.; Negi, J.S.; Rawat, M.S.M.; Pant, G.J.N. Quantification of mineral elements of Rheum emodi Wallr. (Polygonaceae). Biol. Trace Elem. Res. 2010, 138, 293-299. [CrossRef]

71. Negi, J.S.; Singh, P.; Pant, G.J.N.; Rawat, M.S.M. Study on the variations of mineral elements in Swertia speciosa (G. Don). Biol. Trace Elem. Res. 2010, 138, 300-306. [CrossRef]

72. Zeng, X.; Yuan, H.; Dong, X.; Peng, M.; Jing, X.; Xu, Q.; Tang, T.; Wang, Y.; Zha, S.; Gao, M.; et al. Genome-wide dissection of Co-selected UV-B responsive pathways in the UV-B adaptation of Qingke. Mol. Plant 2020, 13, 112-127. [CrossRef]

73. Harborne, J.B.; Williams, C.A. Advances in flavonoid research since 1992. Phytochemistry 2000, 55, 481-504. [CrossRef] 
74. Ganzera, M.; Guggenberger, M.; Stuppner, H.; Zidorn, C. Altitudinal variation of secondary metabolite profiles in flowering heads of Matricaria chamomilla cv. BONA. Planta Med. 2008, 74, 453-457. [CrossRef]

75. Kumari, M.; Joshi, R.; Kumar, R. Metabolic signatures provide novel insights to Picrorhiza kurroa adaptation along the altitude in Himalayan region. Metabolomics 2020, 16, 1-17. [CrossRef]

76. De Oliveira, J.B.; Egipto, R.; Laureano, O.; de Castro, R.; Pereira, G.E.; Ricardo-Da-Silva, J.M. Climate effects on physicochemical composition of Syrah grapes at low and high altitude sites from tropical grown regions of Brazil. Food Res. Int. 2019, 121, 870-879. [CrossRef]

77. Ni, Q.; Wang, Z.; Xu, G.; Gao, Q.; Yang, D.; Morimatsu, F.; Zhang, Y. Altitudinal variation of antioxidant components and capability in Indocalamus latifolius (Keng) McClure Leaf. J. Nutr. Sci. Vitaminol. 2013, 59, 336-342. [CrossRef]

78. Barnes, P.W.; Flint, S.D.; Tobler, M.A.; Ryel, R.J. Diurnal adjustment in ultraviolet sunscreen protection is widespread among higher plants. Oecologia 2016, 181, 55-63. [CrossRef]

79. Rizi, M.R.; Azizi, A.; Sayyari, M.; Mirzaie-Asl, A.; Conti, L. Increased phenylpropanoids production in UV-B irradiated Salvia verticillata as a consequence of altered genes expression in young leaves. Plant Physiol. Biochem. 2021, 167, 174-184. [CrossRef]

80. Rozema, J.; Björn, L.; Bornman, J.; Gaberščik, A.; Häder, D.-P.; Trošt, T.; Germ, M.; Klisch, M.; Gröniger, A.; Sinha, R.; et al. The role of UV-B radiation in aquatic and terrestrial ecosystems-an experimental and functional analysis of the evolution of UV-absorbing compounds. J. Photochem. Photobiol. B Biol. 2002, 66, 2-12. [CrossRef]

81. Solovchenko, A. Significance of skin flavonoids for UV-B-protection in apple fruits. J. Exp. Bot. 2003, 54, 1977-1984. [CrossRef]

82. Shafi, M.; Mansoor, S.; Jan, S.; Singh, D.B.; Kazi, M.; Raish, M.; Al Wadei, M.; Mir, J.I.; Ahmad, P. Variability in Catechin and Rutin contents and their antioxidant potential in diverse apple Genotypes. Molecules 2019, 24, 943. [CrossRef]

83. Rajan, M.; Feba, K.S.; Chandran, V.; Shahena, S.; Mathew, L. Enhancement of rhamnetin production in Vernonia anthelmintica (L.) Willd. cell suspension cultures by eliciting with methyl jasmonate and salicylic acid. Physiol. Mol. Biol. Plants 2020, 26, 1531-1539. [CrossRef]

84. An, J.-P.; Li, R.; Qu, F.-J.; You, C.-X.; Wang, X.-F.; Hao, Y.-J. R2R3-MYB transcription factor MdMYB23 is involved in the cold tolerance and proanthocyanidin accumulation in apple. Plant J. 2018, 96, 562-577. [CrossRef]

85. Neugart, S.; Bumke-Vogt, C. Flavonoid Glycosides in Brassica species respond to UV-B depending on exposure time and adaptation time. Molecules 2021, 26, 494. [CrossRef]

86. Spitaler, R.; Winkler, A.; Lins, I.; Yanar, S.; Stuppner, H.; Zidorn, C. Altitudinal variation of phenolic contents in flowering heads of Arnica montana cv. ARBO: A 3-year comparison. J. Chem. Ecol. 2008, 34, 369-375. [CrossRef]

87. Ohno, A.; Oka, K.; Sakuma, C.; Okuda, H.; Fukuhara, K. Characterization of tea cultivated at four different altitudes using $1 \mathrm{H}$ NMR analysis coupled with multivariate statistics. J. Agric. Food Chem. 2011, 59, 5181-5187. [CrossRef]

88. Berardi, A.E.; Fields, P.D.; Abbate, J.L.; Taylor, D.R. Elevational divergence and clinal variation in floral color and leaf chemistry in Silene vulgaris. Am. J. Bot. 2016, 103, 1508-1523. [CrossRef] [PubMed]

89. Rather, R.A.; Bhagat, M. Quercetin as an innovative therapeutic tool for cancer chemoprevention: Molecular mechanisms and implications in human health. Cancer Med. 2020, 9, 9181-9192. [CrossRef]

90. Chen, A.Y.; Chen, Y.C. A review of the dietary flavonoid, kaempferol on human health and cancer chemoprevention. Food Chem. 2013, 138, 2099-2107. [CrossRef]

91. Mishra, C.B.; Pandey, P.; Sharma, R.D.; Malik, Z.; Mongre, R.K.; Lynn, A.M.; Prasad, R.; Jeon, R.; Prakash, A. Identifying the natural polyphenol catechin as a multi-targeted agent against SARS-CoV-2 for the plausible therapy of COVID-19: An integrated computational approach. Brief. Bioinform. 2021, 22, 1346-1360. [CrossRef]

92. Koirala, N.; Pandey, R.P.; Parajuli, P.; Jung, H.J.; Sohng, J.K. Methylation and subsequent glycosylation of 7,8-dihydroxyflavone. J. Biotechnol. 2014, 184, 128-137. [CrossRef]

93. Sordon, S.; Popłoński, J.; Huszcza, E. Microbial Glycosylation of Flavonoids. Pol. J. Microbiol. 2016, 65, 137-151. [CrossRef]

94. Nie, Y.; Stürzenbaum, S.R. Proanthocyanidins of natural origin: Molecular mechanisms and implications for lipid disorder and aging-associated diseases. Adv. Nutr. 2019, 10, 464-478. [CrossRef]

95. Sandhu, A.K.; Miller, M.G.; Thangthaeng, N.; Scott, T.M.; Shukitt-Hale, B.; Edirisinghe, I.; Burton-Freeman, B. Metabolic fate of strawberry polyphenols after chronic intake in healthy older adults. Food Funct. 2018, 9, 96-106. [CrossRef]

96. Kim, Y.-J. Antimelanogenic and antioxidant properties of gallic acid. Biol. Pharm. Bull. 2007, 30, 1052-1055. [CrossRef]

97. Punia, A.; Chauhan, N.S.; Singh, D.; Kesavan, A.K.; Kaur, S.; Sohal, S.K. Effect of gallic acid on the larvae of Spodoptera litura and its parasitoid Bracon hebetor. Sci. Rep. 2021, 11, 1-11. [CrossRef]

98. Rubin-Aguirre, A.; Saenz-Romero, C.; Lindig-Cisneros, R.; Del-Rio-Mora, A.; Tena-Morelos, C.; Campos-Bolaños, R.; Del-Val, E. Bark beetle pests in an altitudinal gradient of a Mexican managed forest. For. Ecol. Manag. 2015, 343, 73-79. [CrossRef]

99. Rudrappa, T.; Choi, Y.S.; Levia, D.F.; Legates, D.R.; Lee, K.H.; Bais, H.P. Phragmites australisroot secreted phytotoxin undergoes photo-degradation to execute severe phytotoxicity. Plant Signal. Behav. 2009, 4, 506-513. [CrossRef]

100. Neelam; Khatkar, A.; Sharma, K.K. Phenylpropanoids and its derivatives: Biological activities and its role in food, pharmaceutical and cosmetic industries. Crit. Rev. Food Sci. Nutr. 2020, 60, 2655-2675. [CrossRef]

101. Zhang, S.; Huang, Y.; Li, Y.; Wang, Y.; He, X. Anti-neuroinflammatory and antioxidant phenylpropanoids from Chinese olive. Food Chem. 2019, 286, 421-427. [CrossRef]

102. Kolaj, I.; Liyanage, S.I.; Weaver, D.F. Phenylpropanoids and Alzheimer's disease: A potential therapeutic platform. Neurochem. Int. 2018, 120, 99-111. [CrossRef] 
103. Kishorekumar, R.; Bulle, M.; Wany, A.; Gupta, K.J. An overview of important enzymes involved in nitrogen assimilation of plants. Springer Protoc. Handb. 2020, 2057, 1-13. [CrossRef]

104. Taiz, L.; Zeiger, E. Chapter 12: Assimilation of mineral nutrients. In Plant Physiology, 4th ed.; Sinauer Associates, Inc.: Sunderland, MA, USA, 2006; pp. 265-266. ISBN 9780878938568.

105. Rehman, H.U.; Alharby, H.F.; Bamagoos, A.A.; Abdelhamid, M.T.; Rady, M.M. Sequenced application of glutathione as an antioxidant with an organic biostimulant improves physiological and metabolic adaptation to salinity in wheat. Plant Physiol. Biochem. 2021, 158, 43-52. [CrossRef] [PubMed]

106. Schenck, C.; Maeda, H.A. Tyrosine biosynthesis, metabolism, and catabolism in plants. Phytochemistry 2018, 149, 82-102. [CrossRef] [PubMed]

107. Winter, G.; Todd, C.D.; Trovato, M.; Forlani, G.; Funck, D. Physiological implications of arginine metabolism in plants. Front. Plant Sci. 2015, 6, 534. [CrossRef]

108. Preczenhak, A.P.; Orsi, B.; Lima, G.P.P.; Tezotto-Uliana, J.V.; Minatel, I.O.; Kluge, R. Cysteine enhances the content of betalains and polyphenols in fresh-cut red beet. Food Chem. 2019, 286, 600-607. [CrossRef]

109. Wang, Y.; Xiong, F.; Nong, S.; Liao, J.; Xing, A.; Shen, Q.; Ma, Y.; Fang, W.; Zhu, X. Effects of nitric oxide on the GABA, polyamines, and proline in tea (Camellia sinensis) roots under cold stress. Sci. Rep. 2020, 10, 1-10. [CrossRef]

110. Anwar, A.; She, M.; Wang, K.; Riaz, B.; Ye, X. Biological roles of Ornithine Aminotransferase (OAT) in plant stress tolerance: Present progress and future perspectives. Int. J. Mol. Sci. 2018, 19, 3681. [CrossRef] [PubMed]

111. Xiaochuang, C.; Chu, Z.; Lianfeng, Z.; Junhua, Z.; Hussain, S.; Lianghuan, W.; Qianyu, J. Glycine increases cold tolerance in rice via the regulation of $\mathrm{N}$ uptake, physiological characteristics, and photosynthesis. Plant Physiol. Biochem. 2017, 112, 251-260. [CrossRef]

112. Han, M.; Zhang, C.; Suglo, P.; Sun, S.; Wang, M.; Su, T. L-Aspartate: An essential metabolite for plant growth and stress acclimation. Molecules 2021, 26, 1887. [CrossRef]

113. Arruda, P.; Barreto, P. Lysine Catabolism through the Saccharopine pathway: Enzymes and intermediates involved in plant responses to abiotic and biotic stress. Front. Plant Sci. 2020, 11, 587. [CrossRef]

114. Joshi, V.; Jander, G. Arabidopsis Methionine $\gamma$-Lyase is regulated according to Isoleucine Biosynthesis needs but plays a subordinate role to Threonine Deaminase. Plant Physiol. 2009, 151, 367-378. [CrossRef]

115. Zhao, C.; Zayed, O.; Yu, Z.; Jiang, W.; Zhu, P.; Hsu, C.-C.; Zhang, L.; Tao, W.A.; Lozano-Durán, R.; Zhu, J.-K. Leucine-rich repeat extensin proteins regulate plant salt tolerance in Arabidopsis. Proc. Natl. Acad. Sci. USA 2018, 115, 13123-13128. [CrossRef]

116. Gipson, A.; Morton, K.J.; Rhee, R.J.; Simo, S.; Clayton, J.A.; Perrett, M.E.; Binkley, C.G.; Jensen, E.L.; Oakes, D.L.; Rouhier, M.F.; et al. Disruptions in valine degradation affect seed development and germination in Arabidopsis. Plant J. 2017, 90, 1029-1039. [CrossRef]

117. Sazuka, T.; Kamiya, N.; Nishimura, T.; Ohmae, K.; Sato, Y.; Imamura, K.; Nagato, Y.; Koshiba, T.; Nagamura, Y.; Ashikari, M.; et al. A rice tryptophan deficient dwarf mutant, $t d d 1$, contains a reduced level of indole acetic acid and develops abnormal flowers and organless embryos. Plant J. 2009, 60, 227-241. [CrossRef]

118. Thevenet, D.; Pastor, V.; Baccelli, I.; Balmer, A.; Vallat, A.; Neier, R.; Glauser, G.; Mauch-Mani, B. The priming molecule $\beta$-aminobutyric acid is naturally present in plants and is induced by stress. New Phytol. 2017, 213, 552-559. [CrossRef] [PubMed]

119. Balmer, A.; Glauser, G.; Mauch-Mani, B.; Baccelli, I. Accumulation patterns of endogenous $\beta$-aminobutyric acid during plant development and defence in Arabidopsis thaliana. Plant Biol. 2018, 21, 318-325. [CrossRef] [PubMed]

120. Kuczyńska, A.; Cardenia, V.; Ogrodowicz, P.; Kempa, M.; Rodriguez-Estrada, M.T.; Mikołajczak, K. Effects of multiple abiotic stresses on lipids and sterols profile in barley leaves (Hordeum vulgare L.). Plant Physiol. Biochem. 2019, 141, 215-224. [CrossRef]

121. Xu, C.; Shanklin, J. Triacylglycerol metabolism, function, and accumulation in plant vegetative tissues. Annu. Rev. Plant Biol. 2016, 67, 179-206. [CrossRef]

122. Wang, Q.; Xu, X.; Cao, X.; Hu, T.; Xia, D.; Zhu, J.; Zhan, X. Identification, classification, and expression analysis of the Triacylglycerol Lipase (TGL) gene family related to abiotic stresses in tomato. Int. J. Mol. Sci. 2021, 22, 1387. [CrossRef] [PubMed]

123. Lafta, A.M.; Fugate, K.K. Metabolic profile of wound-induced changes in primary carbon metabolism in sugarbeet root. Phytochemistry 2011, 72, 476-489. [CrossRef]

124. Zhang, Y.-G.; Kan, H.; Chen, S.-X.; Thakur, K.; Wang, S.; Zhang, J.-G.; Shang, Y.-F.; Wei, Z.-J. Comparison of phenolic compounds extracted from Diaphragma juglandis fructus, walnut pellicle, and flowers of Juglans regia using methanol, ultrasonic wave, and enzyme assisted-extraction. Food Chem. 2020, 321, 126672. [CrossRef]

125. Sun, L.; Isaak, C.K.; Zhou, Y.; Petkau, J.C.; Karmin, O.; Liu, Y.; Siow, Y. Salidroside and tyrosol from Rhodiola protect H9c2 cells from ischemia/reperfusion-induced apoptosis. Life Sci. 2012, 91, 151-158. [CrossRef]

126. Fan, H.; Sun, L.; Yang, L.; Zhou, J.; Yin, P.; Li, K.; Xue, Q.; Li, X.; Liu, Y. Assessment of the bioactive phenolic composition of Acer truncatum seed coat as a byproduct of seed oil. Ind. Crop. Prod. 2018, 118, 11-19. [CrossRef]

127. Ministry of Environmental Protection of China. Solid waste-determination of metals-inductively coupled plasma mass spectrometry (ICP-MS). In National Environmental Protection Standards of the People's Republic of China (HJ766-2015); China Environmental Press: Beijing, China, 2015; ISBN 135111068.

128. Fan, W.; Li, B.; Tian, H.; Li, X.; Ren, H.; Zhou, Q. Metabolome and transcriptome analysis predicts metabolism of violet-red color change in Lilium bulbs. J. Sci. Food Agric. 2021. [CrossRef] 